Florida International University FIU Digital Commons

$12-8-2015$

\title{
A Randomized Comparison of Two Instructional Sequences for Imitation Intervention for Children with Autism Spectrum Disorders
}

Elaine Espanola

eespanol@fiu.edu

DOI: $10.25148 /$ etd.FIDC000232

Follow this and additional works at: https://digitalcommons.fiu.edu/etd

Part of the Applied Behavior Analysis Commons, and the Developmental Psychology Commons

\section{Recommended Citation}

Espanola, Elaine, "A Randomized Comparison of Two Instructional Sequences for Imitation Intervention for Children with Autism Spectrum Disorders" (2015). FIU Electronic Theses and Dissertations. 2490.

https://digitalcommons.fiu.edu/etd/2490 


\section{FLORIDA INTERNATIONAL UNIVERSITY}

Miami, Florida

\section{A RANDOMIZED COMPARISON OF TWO INSTRUCTIONAL SEQUENCES FOR IMITATION INTERVENTION FOR CHILDREN WITH AUTISM SPECTRUM DISORDERS}

A dissertation submitted in partial fulfillment of

the requirements for the degree of

DOCTOR OF PHILOSOPHY

in

PSYCHOLOGY

by

Elaine Espanola

2016 
To: Dean Michael R. Heithaus

College of Arts, Sciences and Education

This dissertation, written by Elaine Espanola, and entitled A Randomized Comparison of Two Instructional Sequences for Imitation Intervention for Children with Autism Spectrum Disorders, having been approved in respect to style and intellectual content, is referred to you for judgment.

We have read this dissertation and recommend that it be approved.

$\begin{array}{r}\hline \text { Bethany C. Reeb-Sutherland } \\ \hline \text { Leslie D. Frazier } \\ \hline \text { Anibal Gutierrez, Jr., Major Professor }\end{array}$

Date of Defense: December 8, 2015.

The dissertation of Elaine Espanola is approved.

Dean Michael R. Heithaus

College of Arts, Sciences and Education

Andrés G. Gil

Vice President for Research and Economic Development and Dean of the University Graduate School

Florida International University, 2016 
(C) Copyright 2016 by Elaine Espanola

All rights reserved. 


\section{DEDICATION}

This dissertation is dedicated to my husband and my parents. It is very difficult to express the depth of my gratitude for your continued support, your words of encouragement and your resounding love. This incredible journey would not have been possible without you. Thank you for believing in me, I am forever grateful. 


\section{ACKNOWLEDGMENTS}

I would like to express my gratitude to the members of my committee for their guidance and support. I would like to especially thank Dr. Anibal Gutierrez, my mentor and committee chairman, who shared his knowledge and expertise and also provided me with support and encouragement when I most needed it.

I am also grateful to my research assistants and my fellow graduate students in the Behavior Analysis Autism Research lab, who all played an important role in the completion of my dissertation and made this journey an unforgettable experience. I would also like to thank the Florida Education Fund, not only for their monetary support, but also for being a great source of inspiration, support and encouragement. 
ABSTRACT OF THE DISSERTATION

A RANDOMIZED COMPARISON OF TWO INSTRUCTIONAL SEQUENCES FOR IMITATION INTERVENTION FOR CHILDREN WITH AUTISM SPECTRUM DISORDER by

Elaine Espanola

Florida International University, 2016

Miami, Florida

Professor Anibal Gutierrez, Major Professor

Purpose: The aim of this study was to determine differences in effectiveness and rate of skill acquisition between a recently developed and empirically validated instructional sequence, Motor and Vocal Imitation Assessment (MVIA), and a commonly used instructional sequence in a curriculum guide, Verbal Behavior Milestones Assessment and Placement Program (VB-MAPP).

Methods: Children with ASD were randomly assigned to two treatment groups to determine difference in imitation performance. The treatment group followed the instructional sequence proposed in the MVIA. The comparison group followed the instructional sequence proposed in the VB-MAPP. Initial levels of imitation were assessed via the MVIA. The intervention consisted of discrete trial training (DTT). A trained therapist presented a fixed number of stimuli in massed trial format. Prompted and unprompted imitative responses were reinforced using edibles. A most-to-least with a progressive time delay prompting strategy was used to help the learner engage in the target response. 
Results: Participants in the MVIA treatment group had significantly more skill acquisition than participants in the VB-MAPP comparison group. Participants in the MVIA treatment group also acquired these skills more efficiently, spent less time on skills that never reached mastery and demonstrated higher levels of responding. Additionally, pre-treatment imitation was found to predict autism severity and expressive language.

Conclusions: These results indicate that the MVIA protocol provides an appropriate sequence ordered from simple to complex for selecting targets for intervention. These findings suggest that organizing and sequencing skills in increasing difficulty, as with the MVIA protocol, leads to more appropriate target selection. Targeting skills that are appropriate for the child's current skill level, in turn leads to more effective and efficient intervention. Results also replicate previous findings that demonstrate that imitation performance plays a critical role in other areas of development.

Keywords: autism, imitation, early intervention, instructional sequence, assessment 


\section{TABLE OF CONTENTS}

CHAPTER PAGE

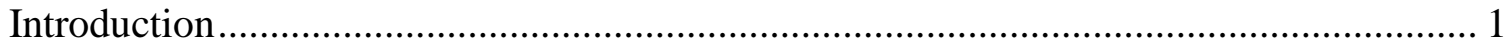

Autism Spectrum Disorders: A Review of the literature .......................................... 2

Autism Symptomology. ............................................................................... 3

Prognosis, Causes, and Risk Factors............................................................... 4

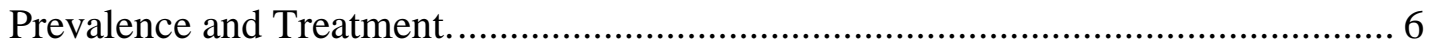

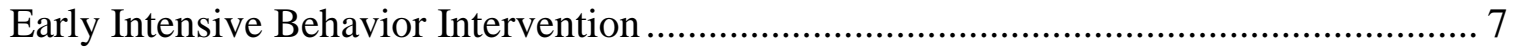

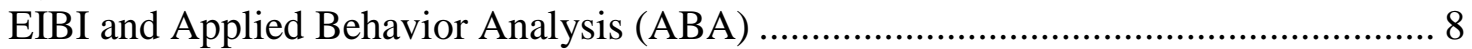

Unit of Analysis: Three-term contingency. ........................................................ 9

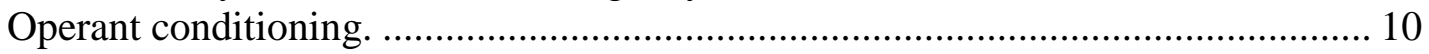

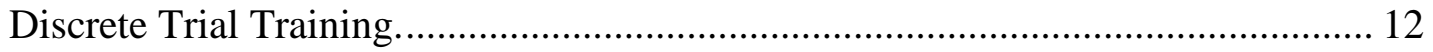

EIBI and Selection of treatment goals. .......................................................... 13

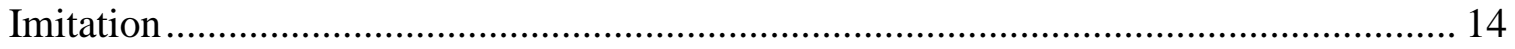

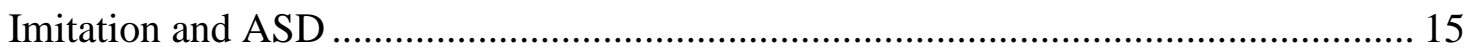

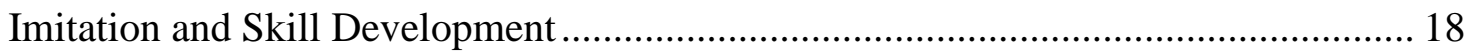

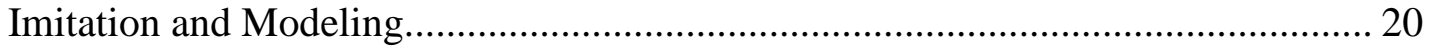

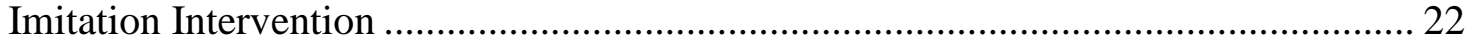

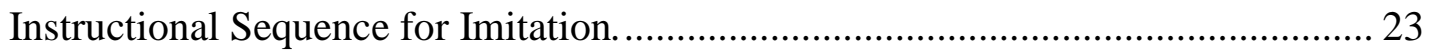

Hierarchy of Imitation Tasks. .......................................................................... 26

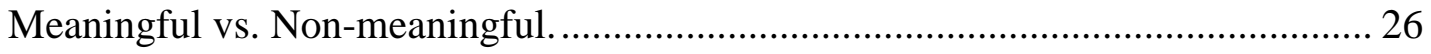

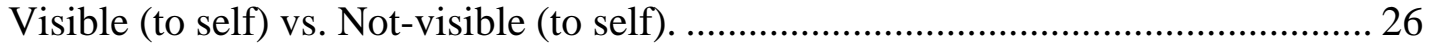

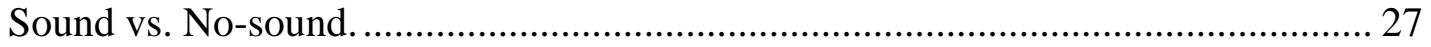

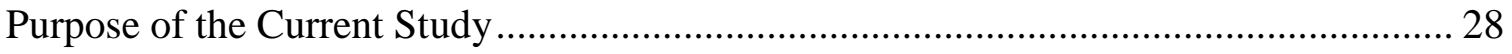

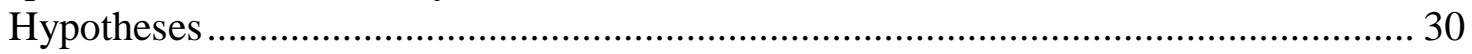

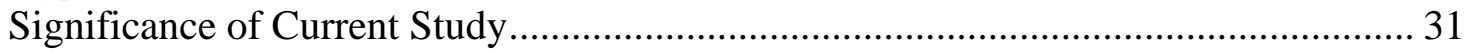

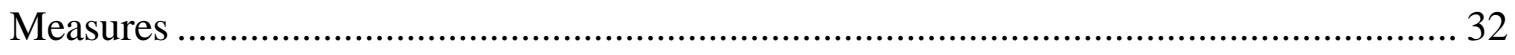

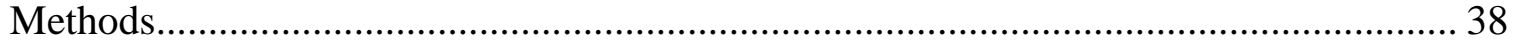

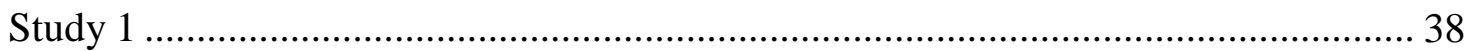

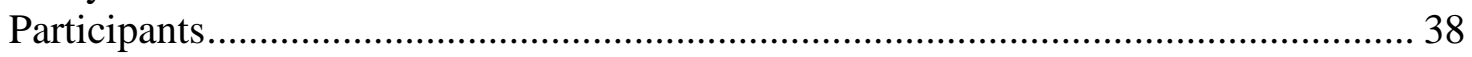

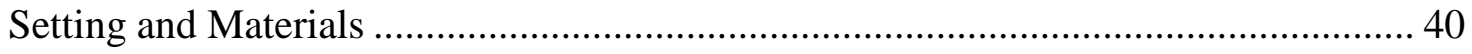

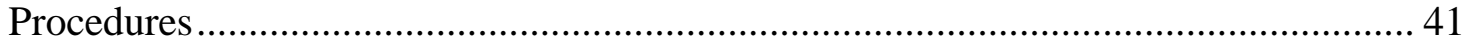

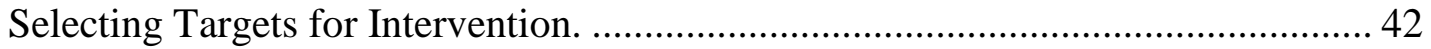

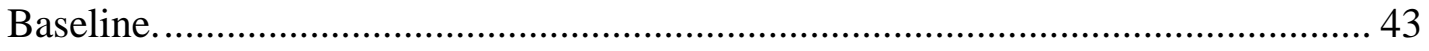

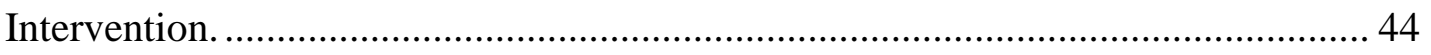

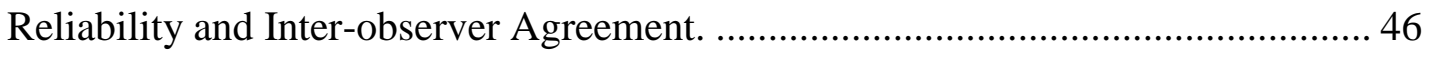

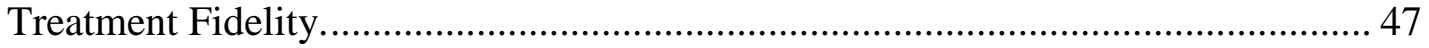

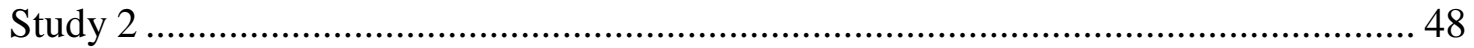

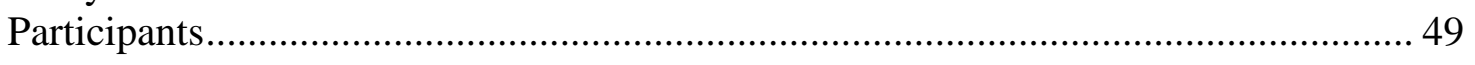

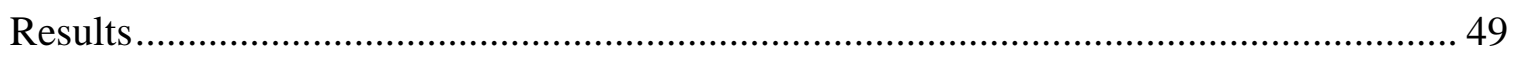




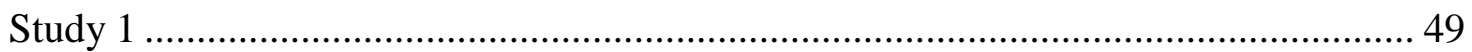

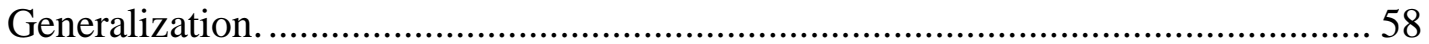

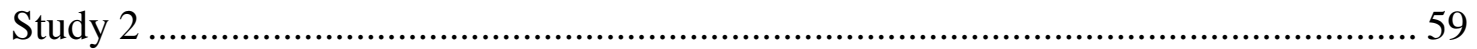

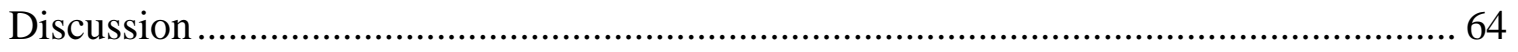

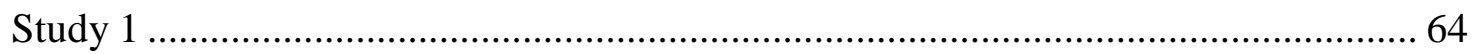

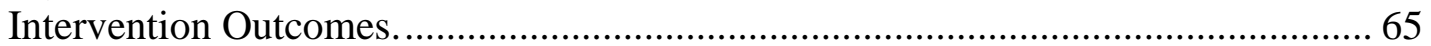

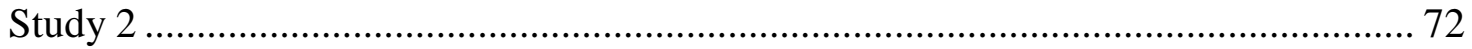

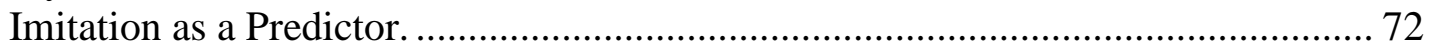

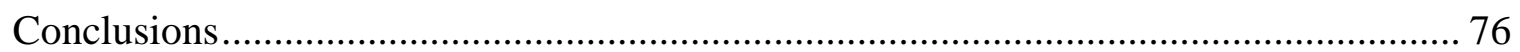

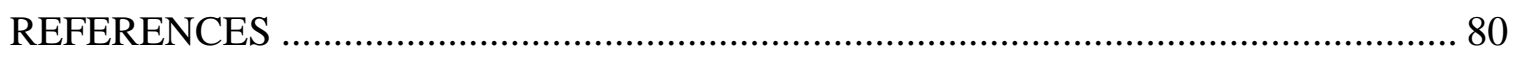

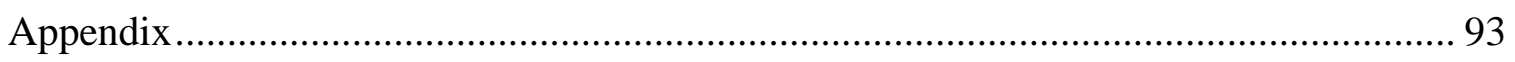

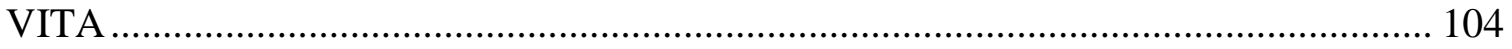




\section{LIST OF TABLES}

TABLE

PAGE

1. Study 1: Summary of Sample Characteristics

2. Study 2: Summary of Sample Characteristics

3. Correlations for Imitation Scales................................................... 50

4. Descriptive Statistics for the Motor and Vocal Imitation Assessment................. 51

5. One Way ANOVA Results for Differences Between

MVIA Treatment Group and the VBMAPP Comparison Group......................... 55

6. Intervention Outcomes by Imitation Type for

the MVIA Treatment Group and the VBMAPP Comparison Group..................... 58

7. Correlations Between MVIA Scores and Developmental Measures

for Children with ASD ........................................................... 60 


\section{LIST OF FIGURES}

FIGURE

PAGE

1. Sequence of imitation skills for the VB-MAPP and the MVIA..................... 43

2. Prompting hierarchy and procedures for moving through the hierarchy............. 45

3. Graph of MVIA imitation performance scores across all participants............. 52

4. Cumulative graph representing generalization of imitation skills

for the MVIA treatment group and the VB-MAPP comparison group.......................... 59

5. Scatter plot representing the strong negative correlation

between imitation and autism severity, as measured by the ADOS .................... 62

6. Scatter plot representing the strong positive correlation

between imitation and expressive language, as measured by the Mullen

64 


\section{Introduction}

Autism Spectrum Disorder (ASD) is a multifaceted neurodevelopmental disorder that affects various domains of functioning. Communication and social skills are particularly affected domains in children with ASD (American Psychological Association, 2012). However, research has demonstrated that children with ASD that participate in Early Intensive Behavior Intervention (EIBI) programs have positive outcomes and exhibit improvements in communication and social skills (Warren et al., 2011). A critical component in the success of EIBI programs is the selection of appropriate skills (Green, Brennan, \& Fein, 2002).

Imitation, amongst other socio-communicative skills, is frequently targeted for intervention during EIBI programs. Imitation is often targeted for intervention because it serves as the foundation for the development of other skills and is associated with positive outcomes in children with ASD (Kasari, Paparella, Freeman, \& Jahromi, 2008). Despite the importance of imitation, researchers and clinicians lack an empirically validated protocol for selecting imitation targets (Ledford \& Wolery, 2011). Instead clinicians rely on protocols in published curricula that contain sequence of imitation skills. Although these published curricula are helpful, it is important to note that they have never been directly evaluated. The order of imitation skills suggested in these curricula may not reflect a true hierarchy of complexity, potentially resulting in the selection of targets that are not appropriate for the learner's current skill level. Selecting appropriate targets for intervention is an indispensable component in formulating effective and efficient interventions. Choosing targets in increasing complexity may lead to an increase in skill acquisition and may result in more appropriate use of valuable 
resources and time. Therefore, it may be important to establish a protocol that can guide target selection across a sequence of skills organized from simple to complex.

The present study will evaluate differences in performance in children with ASD who receive interventions targeting imitation. Children will be randomly assigned into two groups: 1) Motor and Vocal Imitation Assessment treatment group (MVIA);

2) Verbal Behavior Milestones Assessment and Placement Program comparison group (VB-MAPP). The intervention procedures will be the same for both groups, the only controlled difference will be the protocol used to select targets for intervention.

The Motor and Vocal Imitation Assessment protocol provides a sequence of skills that is described in the current literature and has been empirically validated (Espanola, 2014), while the Verbal Behavior Milestones Assessment and Placement Program (VB-MAPP) is a published curriculum that is commonly used and consists of a sequence of skills that has not been evaluated.

\section{Autism Spectrum Disorders: A Review of the literature}

The Diagnostic and Statistical Manual of Mental Disorders (5th ed.; DSM-5; American Psychiatric Association, 2013) states that ASD is characterized by deficits in communication, social interaction, and the presence of restricted, repetitive patterns of behavior, interests, or activities. The diagnostic definition of ASD was updated in 2013; this definition was simplified in order to encompass the different subgroups of the disorder. Autism, PDD-NOS, and Asperger's disorder are now redefined as one disorder, ASD (Matson, Kozlowski, Hattier, Horovitz, \& Sipes, 2012). Studies have concluded that there are few differences between these subgroups of disorders and support the unification of subgroups into a single diagnosis (Macintosh \& Dissanayake, 2004). 
Autism Symptomology. A variety of studies have focused on identifying when ASD emerges and when symptoms associated with ASD become evident (Guthrie, Swineford, Nottke, \& Wetherby, 2013; Seneff, Davidson, \& Liu, 2012; Ventola, Saulnier, Steinberg, Chawarska, \& Klin, 2014). Understanding when ASD emerges is essential not only for conceptualizing ASD but also has strong clinical implications for screening, diagnosis, and intervention. Studies have not been able to conclusively demonstrate when ASD emerges. Some research suggests that autism may have a "presymptomatic period.” The period is characterized by abnormal brain circuitry and a lack of behavioral manifestation of symptoms (e.g., Lewis, 2004). Symptoms become evident at different times, in other words, these behavioral manifestations occur along a continuum. For instance, some children may display a developmental plateau that is evident by a lack of progress and other children may exhibit a loss of previously developed skills. However, there are children who exhibit mixed characteristic, these children demonstrate both an early deficit and a regression of skills (Ozonoff, Heung, Byrd, Hansen \& Hertz-Picciotto, 2008).

Manifestation of behavioral symptoms of ASD is most evident to caregivers when children are 18 months of age and there is an increase in social communication demands (Horovitz \& Matson, 2010). Researchers have been working towards early identification by investigating when development in ASD diverges from typical development (e.g., Ozonoff et al., 2014; Sacrey, Bryson, \& Zwaigenbaum, 2013; Wan et al., 2013). A recent study examined when development in ASD diverges from typical development by conducting a prospective longitudinal design. The design was used to evaluate behavioral differences in 294 high-risk infants and 116 low-risk infants. Participants were evaluated 
at $6,12,18,24$, and 36 months of age. At the conclusion of the study children's outcomes were classified as ASD, typically developing (TD), or Non-TD (described as elevated Autism Diagnostic Observation Schedule [ADOS] score, low mental age scores, or both). The results showed that behavioral manifestations associated with the broader autism phenotype are not present at birth but are evident by the first birthday and affect development in multiple domains, with particular prominent delays in socialcommunication domains (Ozonoff et al., 2014).

Symptoms that have lead to early identification include: (1) delayed or irregular visual examination and fixations; (2) the presence of repetitive patterns of object exploration; (3) an absence of intentional communicative responses (4) deficient phonemic growth; (5) an absence of coordinated gaze, affect, and voice during interactions with others; (6) inconsistent eye contact; (7) and atypical social interest, and engagement (Bryson et al., 2007).

Prognosis, Causes, and Risk Factors. For most, ASD is a lifelong disorder, with symptoms persisting from early childhood through late adulthood. (Rogers, 2004; Smith, 1999) These symptoms have been recently categorized into three levels. The levels are defined by the amount of support the individual will require. For instance, level 2 denotes “substantial support” while level 3 denotes "very substantial support” (American Psychiatric Association, 2013). Additionally, the disorder occurs within a spectrum, this means that symptomology is heterogeneous across individuals and that no two individuals exhibit the exact same behavioral manifestations.

There have been a plethora of studies that have sought to identify the cause of ASD (e.g., Chaste \& Leboyer, 2012; Landrigan, Lambertini, \& Birnbaum, 2012; 
Ratajczak, 2011; Larsson et al., 2005; Ronald \& Hoekstra, 2014). However, there has been little evidence to implicate any one factor as the major cause. Kanner (1943) was the first to identify ASD and hypothesized that ASD was the product of poor parenting skills. Subsequent twin and family studies countered this hypothesis. Twin and family studies were the major driving force behind the perspective that genes were the main causal factor in ASD. Folstein and Rutter (1977) found that there were significant monozygoticdizygotic (MZ-DZ) differences in concordance and showed that the twins were concordant for a range of social and cognitive impairments. The basic conclusions from various twin and family studies were that: (a) the heritability of ASD is greater than $90 \%$; (b) this heritability goes well past the established symptomology; and (c) it is likely that more than one interacting gene causes ASD (Rutter, 2000). However, researchers have questioned the notion genes are the main causal factor in ASD, proposing that all heritability estimates are confounded by the effect of gene-environment interactions (Simonoff, 2012). The current consensus is that there are multiple genetic and nongenetic etiologies that result in this complex and multifaceted disorder (Simonoff, 2012).

Researchers are actively investigating the multiple and continuous interactions between genetic, biological, and environmental factors that cause ASD. There are various studies that have been able to discern the risk factors that increase the chances of having ASD (Newschaffer et al., 2012). Presently, ASD affects males five times more often than females ( 1 out of 42 boys have ASD, whereas 1 out of 189 girls have ASD; Centers for Disease Control and Prevention [CDC], 2014). Studies have also reliably indicated that children that have family members with ASD are also more likely to be diagnosed with ASD (CDC, 2014; Newschaffer et al., 2012). The probability of diagnosis for a child with 
a sibling who has ASD is as follows: (a) 36\% to 95\% if their identical twin has been diagnosed with ASD; (b) $0 \%$ to 31\% if their fraternal twin has been diagnosed with ASD; or (c) $2 \%$ to $18 \%$ if their older sibling has been diagnosed with ASD (CDC, 2014; Hallmayer et al., 2012). Additionally, presence of genetic or chromosomal conditions, such as fragile $\mathrm{X}$ syndrome or tuberous sclerosis can also increase the risk of being diagnosed with ASD (CDC, 2014; Numis et al., 2011). There are also various environmental risk factors such as premature or low birth weight, maternal stress (prior to 32 weeks of gestation), paternal age, and exposure to heavy metals, pesticides, and specific pharmacological drugs (Dietert, Dietert, \& Dewitt, 2011; Gardener, Spiegelman, \& Buka, 2011).

Prevalence and Treatment. In 2010, according to the CDC's Autism and Developmental Disabilities Monitoring (ADDM) Network approximately 1 in 68 children were identified as having ASD. The current prevalence was more than double the prevalence of children with ASD in 2000 (1 in 150) and nearly a 23\% increase in prevalence of children with ASD in 2008 (1 in 88; Baio, 2012). Overall, the ASD diagnosis has increased 16-fold over the past three decades (Hattier \& Matson, 2012). The dramatic increase in children diagnosed with ASD has led to an increase in funds for autism research. The National Institute of Health (NIH) reported a fivefold increase in autism research funding from 1997 to 2007 (from \$22 million to \$108 million in research). The NIH reported that almost twenty percent of funding for the fiscal year of 2007 was allocated to finding effective treatments to diminish the impact of symptoms associated with ASD and improve social functioning. 
Research has shown that while ASD is a disorder that persists into adulthood (Rogers, 2004; Smith, 1999), young children with ASD that receive applied behavior analytic (ABA) interventions can acquire the communication and social skills required to perform activities that are indispensable for daily life (Helt et al., 2008; Rogers \& Vismara, 2008). Amongst the different treatment methodologies, EIBI programs have received a large amount of empirical support (Eikeseth, 2009; Eldevik et al., 2009; Howlin, Magiati, \& Charman, 2009; Rogers \& Vismara, 2008; Wong et al., 2015).

\section{Early Intensive Behavior Intervention}

Green et al. (2002) stated that EIBI programs are defined by the following features; (a) treatment is typically supervised and implemented by an individual with extensive training and experience in applied behavior analytic procedures and ASD; (b) standardized developmental sequences are used as the basis for selection of treatment goals and objectives; (c) treatment includes a parent implementation or training component; (d) treatment is conducted with a one-to-one therapist and when appropriate, the treatment transitions to small or large group formats; (e) treatment begins at home or center and is gradually extended to other settings; (f) programming is intensive, consisting of 20 to 30 hours of highly structured sessions per week; (g) with treatment lasting an average of two to three years; and (h) with children entering the intervention before the age of 4 .

Early Intensive Behavior Intervention programs have led to moderate gains in cognitive performance, social and language skills, and adaptive behaviors in children 4 years and under diagnosed with ASD (Warren et al., 2011). For instance, children with ASD who participated for 2 years in an EIBI program with the Early Start Denver Model 
(ESDM) exhibited improved performance in cognition and communication when compared to community samples of children with ASD (Dawson et al., 2010). Moreover, results from recent EEG studies measuring event-related potentials and spectral power suggest that toddlers who participated in EIBI programs had normalized patterns of brain responses in areas associated with changes in social behavior during later childhood (Dawson et al., 2012).

These findings affirm the substantial impact EIBI programs can have on children with ASD. It is important to underscore that these interventions are costly and entail a great deal of resources. These interventions approximately cost $\$ 40,000$ to $\$ 60,000$ per child a year (Amendah, Grosse, Peacock, \& Mandell, 2011) and entail 20-30 hours per week of one-to-one and highly structured treatment. Therefore, it is critical that researchers ascertain the factors that produce rapid and lasting learning. Efficient treatment packages should result in a decrease in the resources (e.g., cost, staff) and time that are necessary to attain positive outcomes.

\section{EIBI and Applied Behavior Analysis (ABA)}

One major aspect of EIBI programs is the use of ABA to bring about socially significant change (Green et al., 2002). Baer, Wolf, and Risley (1968) define ABA as the "process of systematically applying interventions based upon the principles of learning theory to improve socially significant behaviors to a meaningful degree, and to demonstrate that the interventions employed are responsible for the improvement in behavior” (p. 91). The principles of learning theory propose that behavior change can be better understood by looking at the relations between observable events (behavior) and its environmental determinants (stimuli). Therefore, the main engine for understanding 
behavior change in ABA is the three-term contingency and the integral concept of reinforcement. The three-term contingency looks at the functional relations between the antecedent stimulus, the response, and the consequence. This functional unit of analysis allows for systematic manipulation of these variables and gives researchers the ability to predict and control responses (Cooper, Heron, \& Heward, 2007).

Unit of Analysis: Three-term contingency. The three-term contingency consist of the antecedent stimulus (A), the behavior (B), and the consequence (C; Glenn, Ellis, \& Greenspoon, 1992). The three-term contingency allows for a comprehensive understanding, control, and prediction of behavior. It underscores the importance of behavior as a function of the circumstances that precede and succeed it. Each component is defined by its relationship to the other. Therefore, no one component alone can explain behavior; rather, it is the relationship between them that allows us to understand behavior.

A behavior can be defined as any observable and measurable act that an organism engages in and that is under the control of an environmental stimulus (Cooper et al., 2007). Operant behavior is defined by its history of consequences. Operant behavior is best understood by the preceding (antecedent stimulus) and subsequent stimuli (consequence) that control it. Antecedent environmental conditions that occur prior to the target behavior are essential in learning and motivation. From an operant conditioning paradigm, a stimulus is any environmental event that changes or influences the response of an organism. A stimulus that indicates that a reinforcer is available for a specific response is called a discriminative stimulus (SD). In other words, the SD is an antecedent stimulus that evokes a behavior by signaling that a response, if emitted, will likely be 
reinforced (Johnston \& Pennypacker, 2009). Stimulus control is acquired when a behavior is said to occur more often in the presence of the stimulus than in the absence. For instance, a child may learn that crying in the presence of father results in access to preferred items but crying in the presence of mother does not. Therefore, in the future they may only cry for access to preferred items when father is present but not when mother is present. Simply stated, stimulus control describes a situation in which a learner is able to distinguish or discriminate under which circumstances a behavior is likely to come into contact with a reinforcer or not.

All behaviors occur under a set of circumstances, consequences are the environmental conditions that follow a behavior. These conditions can either increase or decrease the probability of a response. A reinforcer is a stimulus that when delivered contingent upon a response, increases the probability of that response in the future (Johnston \& Pennypacker, 2009). Moreover, a reinforcer is a stimulus that when removed contingent upon a response, decreases the probability of that response in the future. In both instances a reinforcer is defined by its ability to affect some attribute of the response, that is, by its function. Therefore, when a reinforcer is delivered contingent upon a response but it is no longer affecting some attribute of the response, it is no longer a reinforcer but simply a neutral stimulus. The same conceptualization is used for punishers. A punisher is a stimulus that when delivered contingent upon a response, decreases the likelihood of a response and when removed contingent upon a response, increase the likelihood of a response (Johnston \& Pennypacker, 2009).

Operant conditioning. Operant conditioning defines the circumstances that bring about significant changes in behavior. Operant conditioning refers to learning that occurs 
through reinforcement and punishment (Cooper et al., 2007). Operant conditioning differs from respondent conditioning in that behaviors are not elicited by the stimuli that precede them but rather are influenced by stimulus changes that follow the behavior. Reinforcement denotes an increase in the future probability of a behavior while punishment denotes a decrease in the future probability of a behavior. Reinforcement is defined as a process whereby the future probability of a response is strengthened via the presentation of a reinforcer or the removal of an aversive stimulus (Skinner, 1953). There are two types of reinforcement: positive reinforcement and negative reinforcement. Positive reinforcement will be the main focus of this study.

Positive reinforcement has been recognized as one of the "most important and most widely applied” concepts in behavior analysis (Cooper et al., 2007, p. 258). Positive reinforcement takes place when the probability of a behavior increases contingent upon the presentation of a positive reinforcer. Reinforcement describes a process whereby the future probability responding increases while a positive reinforcer denotes a stimulus whose contingent presentation results in an increase in the future probability of responding.

It is critical to understand that reinforcement describes an empirically demonstrated functional relationship between a stimulus and a response. Thus, reinforcement is only said to have taken place if there is evidence of an increase in some dimension of the behavior (e.g., frequency, duration, magnitude). There are two factors in particular that predict the effectiveness of reinforcement: the immediacy of the reinforcement and the motivation at the time of the behavior. To safeguard against a significant loss of effect, a positive reinforcer should be presented immediately after the 
response (Mazur, 2000). Immediate presentation prevents the unintentional reinforcement of other behaviors that may occur during the delay, as the behavior that is temporally closest to the presentation of the positive reinforcer will be the one increased. The level of motivation at the time of the behavior can also influence reinforcement. For instance, a cookie may not be an effective positive reinforcer if the person is not hungry because they just ate an entire jar of cookies.

Discrete Trial Training. The three-term contingency allows clinicians to breakdown tasks into their basic components in order to effectively create opportunities for learning. This unit of analysis is the foundation and conceptual backbone of Discrete Trial Training (DTT). Discrete Trial Training is one of the many empirically validated instructional techniques used within ABA (Vismara \& Rogers, 2010). Discrete Trial Training has been particularly useful in teaching a variety of skills to children with ASD and has been remarkably valuable in teaching novel or complex behaviors to children with ASD (e.g., Boyle \& Lutzker, 2005). Discrete Trial Training is an adult directed technique in which a trained therapist presents a fixed number of stimuli in massed trial format (Smith, 2001). A trial will typically consist of an antecedent stimulus, a prompting strategy, the target behavior, the consequence, and an inter-trial period (Green, 2001). When a novel behavior is being taught, the therapist will usually present a discriminative stimulus (e.g., "do this” paired with modeling touching nose) followed by a prompt (e.g., physically guiding the learners hand to their nose), and then a consequence. Prompts are systematically faded until the learner can engage in the response without any supplemental assistance or guidance. The use of this simplified instructional unit allows 
learners to access many opportunities for learning (average of 12 per minute) which results in quick skill acquisition (Smith, 2001).

EIBI and Selection of treatment goals. Identifying targets for intervention is an integral element of a successful EIBI program. Researchers propose that early targets should be 'pivotal' skills, or abilities that significantly affect several areas of development such as language and social functioning (Warreyn, Paelt, \& Roeyers, 2014). Imitation, joint attention, and play are three pivotal skills that are often targeted for intervention (e.g., Miniscalco, Rudling, Rastam, Gillberg, \& Johnels, 2014; Pickard \& Ingersoll, 2015). Primarily because these core socio-communication skills are linked to later acquisition of communication and social skills, and general cognitive development (Charman et al., 2000; Charman et al., 2003; Toth, Munson, Meltzoff, \& Dawson, 2006). For instance, Poon, Watson, Baranek, and Poe (2011) conducted a retrospective video analysis to study the degree to which imitation, joint attention, and play behaviors are linked to the development of later communication and intellectual functioning in children with ASD. This study showed that children who engaged in higher level of imitation, joint attention, and object play throughout infancy were more likely to have superior communication and intellectual skills in the preschool and early school age years (Poon et al., 2011). These results concur with previous and subsequent research (McEwen et al., 2007; Young et al., 2011), and bolster the notion that socio-communication skills should be the targeted for treatment as early as possible (Kasari et al., 2008; National Research Council, 2001). The main focus of the present dissertation will be interventions specifically targeting imitation. 


\section{Imitation}

Imitation is a critical skill that children use to learn from their environment (Over \& Carpenter, 2013; Plavnick \& Hume, 2013; Rosales-Ruiz \& Baer, 1997). The word imitation is often used to refer to behavior that is caused by and looks similar to another behavior. Imitation emphasizes that relationship between two behaviors. This relationship between two behaviors assumes that the first behavior sets the occasion for a matching behavior to occur (Jones, 2007). More specifically, Baer, Peterson, and Sherman (1967) stated that a behavior is imitative if it "temporally follows a behavior demonstrated by someone else, called a model, and if its topography is functionally controlled by the topography of the model's behavior” (p. 405).

Gewirtz (1969) proposed that in typically developing infants the first imitative response takes place by chance, with some assistance or direct training. Extrinsic reinforcement from the environment maintains and strengthens this response, but punishes dissimilarity. This trial and error process continues, and once several imitative responses become part of the individual's repertoire, a class of diverse but functionally equivalent behaviors are learned and maintained by extrinsic reinforcement on an intermittent schedule. Through this trial and error process, different environmental stimuli become discriminative, in that they will cue the learner about the availability of reinforcement for imitative responses.

Imitation, from a behavioral perspective, is a type of response class (Young, Krantz, McClannahan, \& Poulson, 1994). A response class is defined as a set of behaviors that are similar in form and/or function and produce the same consequence. Greetings are an example of a response class. For instance, an individual may say "hi,” 
may say "hello,” or may wave to greet another person. These behaviors are not topographically similar but serve the same function. Comparatively, imitative responses may not all be topographically similar (e.g., imitate touching nose, imitate clapping etc.), but have similar functions.

\section{Imitation and ASD}

Studies suggest that difficulty with imitation tasks are evident in children with ASD as young as 24 months of age (Rogers, Hepburn, Stackhouse, \& Wehner, 2003; Stone, Ousley, \& Littleford, 1997; William, Whiten, \& Singh, 2004). In fact, some researchers have come to conclude that poor imitation reflects a substantial deficiency in imitative skills (e.g., Williams et al., 2004). This hypothesis is sustained by studies that show that children with ASD have significantly lower scores on imitation tasks than peers with delayed and typical development (Charman et al., 1997; Smith \& Bryson, 1994) but has not been supported by evidence that indicates that imitation performance is ot significantly different (Stone et al., 1997; Rogers, Young, Cook, Giolzetti, \& Ozonoff, 2010; Young et al., 2011). Overall, the research community agrees that children with ASD have difficulty with imitation tasks.

Researchers have provided a variety of plausible explanations for the differences and similarities in imitation performance between children with ASD and other children. Differences in imitation abilities may be partly explained by ASD-specific traits. For example, when a child with ASD is presented with an imitation task they spend more time looking at the model's action rather than at the model's face (Vivanti et al., 2014). This evidence supports hypotheses that suggest that differences in performance are a due to sensory perception deficits, where deficits in visual recognition of faces and biological 
motion lead to difficulties in the ability to imitate actions (Blake, Turner, Smoski, Pozdol, \& Stone, 2003, Dawson et al., 2002; Dawson, Webb, \& McPartland, 2005). Lack of attention and motivation in children with ASD has also been considered a major factor in differences in performance on imitation tasks. Some studies suggest that poor performance may be in part due to a failure to attend to social stimuli and lack of motivation to engage in imitative responses causes poor performance on imitative tasks (Allen \& Courchesne, 2014; Dawson, Meltzoff, Osterling, Rinaldi, \& Brown 1998; Ingersoll, 2008; Ingersoll, Schreibman, \& Tran, 2003). Difference in performance may also be due to the inherent difficulty of the imitation task, with poor performance reflecting skills that may involve higher motor demands and more cognitive or social processing skills (Stone et al., 1997).

Currently, studies suggest that children with ASD perform better in imitation tasks involving simple one-step actions rather than sequences, objects rather than gestures, meaningful rather than unconventional imitation tasks (Stone et al., 1997), and evoked rather than spontaneous imitation (Ingersoll, 2008). It is critical to understand which skills are more or less difficult for children with ASD to imitate, as information regarding the complexity of imitation may be essential for understanding the nature of imitation and may be helpful when formulating effective interventions. Together, the following studies provide a comprehensive list of characteristics that define the complexity and development of imitation skills.

The Motor Imitation Scale (MIS; Stone et al., 1997) was first used to assess imitation in children ranging from 14 to 39 months of age. The MIS was used to study differences in one-step motor imitation in typically developing (TD) children, children 
with developmental delays (DD) and children with ASD. The MIS assesses two types of imitation: action on object imitation and body imitation (gestures). Object imitation was further categorized as meaningful actions on object (e.g., pushing a car) and nonmeaningful actions on object (e.g., pushing a teacup). The results from the study showed that children in the ASD and TD group performed similarly on imitation tasks. The ASD group performed significantly lower than the DD group on imitation tasks. The assessment indicated that on average all the participants had similar pattern of performance. They all performed better on imitation that consisted of actions on object rather than gestures. Participants also had higher scores on tasks that consisted of meaningful actions on objects versus non-meaningful actions on objects. These results support previous studies (Masur \& Ritz, 1984) and subsequent studies (Rogers et al., 2003; Rogers et al., 2010) showing that imitation tasks can be conceptualized along a continuum of difficulty.

The Imitation Battery (IB; Rogers et al., 2003) assessed imitation in children ranging from 18 to 50 months of age. The IB was first used to assess differences between imitation in children with ASD, fragile X syndrome, and TD children. The IB assessed performance in nine imitation tasks. The tasks are divided into three categories; three tasks are categorized as motor imitation (gestures), three tasks are categorized as action on object imitation, and three tasks are categorized as oral-facial imitation. The IB showed that children with ASD performed differently and had a different pattern of imitation than TD children and children with fragile X syndrome. Children with ASD scored better on actions on object imitation than body imitation, while the opposite was 
true for TD children. TD children and children with ASD had scored better on body imitation than oral-facial imitation.

Jones (2007) studied the development of imitation at 6, 8, 10, 12, 14, 16, 18, and 20 months of age. The assessment was created to assess the pattern of imitation development in TD children. Imitation tasks were categorized into four types: actions that did or did not produce a sound and actions visible (to self) or not visible (to self). The four types of imitation were crossed to achieve eight tasks. For example, behavior one was achieved by crossing actions that did produce a sound with actions that were visible (to self). Results indicate that imitation appears slowly through the first 24 months of age. Imitation of actions that produce a sound develop before imitation of actions that do not produce a sound. Imitation of actions that are visible (to self) develop before actions that not visible (to self). Jones (2007) does not include an ASD sample and does not provide any information regarding possible differences in patterns of development associated with these features of imitation. However, these results provide some insight into features of imitation, such as visibility to self and auditory feedback, which have been overlooked by previous comparison studies.

\section{Imitation and Skill Development}

Early infant imitation appears to facilitate access to various goals; it provides the child with opportunities to contact reinforcers in a social setting and in certain instances it functions as a means of communication between individuals (Nadel, Carchon, Kervella, Marcelli, \& Réserbat- Plantey, 1999; Trevarthen, Kokkinaki, \& Fiamenghi, 1999). Studies investigating the development of both receptive and expressive communication have often explored the role of early predictors such as imitation (e.g., Luyster, Kadlec, 
Carter, \& Tager-Flusberg, 2008; Stone et al., 1997; Stone, \& Yoder, 2001). Studies have shown that imitaton impacts various aspects of language, immediate motor imitation is associated with current language ability, while deferred or delayed imitation contributes to the development and growth of language skills (Toth et al., 2006).

Imitation has also been associated with the development of critical skills, such as: eye contact, visual tracking, approaching and initiating physical contact with others, and the development of a range of facial expressions (Meltzoff, 2005). Amongst social skills, joint attention as related to imitation has been thoroughly researched. In general, joint attention refers to the ability to coordinate attention with the purpose of communicating with others about a third object, person, or event (Mundy, Sigman, \& Kasari, 1990). Previous studies propose that the development of joint attention skills during later infancy begins with understanding individuals as intentional agents (Obhi, \& Sebanz, 2011; Tomasello, 1995), a social cognitive ability that has been closely related to the development of imitation (Meindl \& Cannella-Malone, 2011; Rogers \& Pennington, 1991). Furthermore, as imitation progresses it impacts various social areas developmental; it serves as a foundation for peer interaction, shared experiences, emotions, and thoughts (Sallows \& Graupner, 2005).

The relationship between imitation and the development of other skills has been even more apparent in a recent study that shows a direct relationship between teaching imitation and the development of other skills (Ingersoll, 2012). Ingersoll (2012) investigated the hypothesis that imitation intervention would lead to improvements on social behaviors such as, initiating joint attention and social-emotional functioning in young children with ASD. The results from this study suggest that intervention that 
targets imitation can significantly improve joint attention and social-emotional behaviors in children with ASD. More importantly, these findings were sustained at follow up periods, two to three months after the completion of the intervention. These results replicate previous findings in the literature that show that there is an increase in social skills for children who participate in interventions targeting imitation (e.g., Garfinkle \& Schwartz, 2002; Ingersoll \& Schreibman, 2006). These results further reiterate the importance of imitation skills on the development of socio-communication skills and emphasizes the negative implications that imitation deficits may have on future outcomes.

Imitation and Modeling. The role of imitation in the development of sociocommunication skills is well established (Toth et al., 2006). Often overlooked is the importance of imitation in a clinical setting. Imitation facilitates learning in a clinical setting because it enables the use of modeling as a strategy for teaching new skills. Modeling or the demonstration of the target action, is typically used in a treatment setting as a supplementary stimulus that is presented immediately before or after the behavior to help the learner engage in a target response (MacDuff, Krantz, \& McClannahan, 2001). The efficacy of modeling depends on a learner's imitative repertoire; a learner must be able to imitate the modeled responses in order to come into contact with naturally existing contingencies and reinforcement (Cooper et al., 2007).

Modeling is often used in teaching settings because of its ubiquity in the natural environment, ease of use, and low levels of restrictiveness (Cooper at al., 2007). However, it is essential to emphasize that this prompting strategy is indispensible when teaching early vocal responses. For instance, a therapist teaching a child to request a 
novel item would present the $S^{\mathrm{D}}$, "what do you want?" immediately followed by the model "want milk." If the child matches the modeled response, then they get access to the milk. However, if the child cannot match the modeled response it is impossible to physically manipulate the child's mouth to produce a matching vocal response. Under these circumstances the child never responds so the target behavior never comes into contact with the reinforcer (they never say "want milk," so they never get milk). Thus, the stimulus "what do you want?" never comes to control the target response and the child does not learn to request milk.

Imitation may be particularly significant for children who have limited phonemes or speech sounds. Children who typically have a variety of phonemes or speech sounds may be taught new words via shaping procedures. Shaping procedures establish a desired target behavior by reinforcing a series of successive approximations (Cooper et al., 2007). Previous researchers have investigated different procedures for establishing verbal requests; these studies show that shaping procedures are appropriate and successful but only with children that have partial word approximations (Bourret, Vollmer, \& Trapp, 2004). Partial word approximations are required for shaping procedures because they rely on existing behaviors to shape and create novel behaviors. Therefore, children with limited phonemes or speech sounds may not fully benefit from shaping procedures. There are few alternative for teaching expressive language to children with limited phonemes and speech sounds who cannot imiate vocal response and further underscores the negative impact that imitation deficits can have on tteaching children with few sounds to verbally communicate. 


\section{Imitation Intervention}

A review of imitation intervention research published from 1965 to 2007 was recently conducted for the purpose of identifying the best practices for treatments targeting imitation in children with developmental disorders (Ledford \& Wolery, 2011). Ledford and Wolery (2011) showed that current intervention procedures for imitation are devoid of experimentally validated protocols for target selection. The authors propose this lack of protocol for sequentially ordering imitation tasks could be one of the many factors hindering the success of imitation interventions. The literature within the scope of their review shows that researchers rarely provide reasons for target selection and few consider the developmental course of motor and vocal imitation skills. However, organizing target selection so that it proceeds through a hierchy of increasing complexity may potentially lead to better outcomes in imitation interventions. Therefore, Ledford and Wolery (2011) encourage researchers to create a protocol for target selection to aid treatment development and implementation.

An experimentally grounded and logical protocol for target selection may result in treatment packages that are more effective at bringing about skill acquisition and learning. Interventions that target socio-communication skills largely rely on the sequence organized from simple to complex to guide target selection. For example, when targeting joint attention, targets would proceed through a hierarchy of skills. Initial targets would consist of simple tasks such as responding to social bids (e.g., looking when name is called) and later targets would consist of more difficult tasks like initiating joint attention (e.g., hold out toy for the purpose of sharing the experience with peer). 
Selecting targets based on level of complexity can prevent the misuse of valuable resources, such as time, on targets that are not suitable for the child's current skill level.

Instructional Sequence for Imitation. There are several published curricula, not reviewed by Ledford and Wolery (2011), which provide a theoretically useful hierarchy for a wide variety of skills, including imitation (Leaf \& McEachin, 1999; Lovaas, 1981; Partington, 2008; Sundberg, 2008). Clinicians often use these curricula to guide their selection of short and long terms goals for intervention. However, the hierarchies of skills in these curricula have never been directly tested or analyzed. Thus, it is difficult to determine whether these proposed hierarchies accurately illustrate skills that move from simple to more complex targets. More importantly, the hierarchies of skills in these curricula often do not incorporate recent findings from the literature. Specifically for imitation skills, the hierarchy in these curricula fail to take into consideration various features of imitation tasks that are critical in determining the complexity of the task. For instance, all actions with an object are not equally difficult to imitate. An action on an object that produces a sound is easier to imitate than an action on an object that does not produce a sound (Jones, 2007). Understanding these differences provides a unique perspective on complexity of imitation tasks and allows researchers to make better decisions when formulating and selecting targets for intervention.

The Verbal Behavior Milestones Assessment and Placement Program (VBMAPP) is one of the many published curricula that are widely used in a clinical setting (Sundberg, 2008). The second edition curriculum is founded on B.F. Skinner (1957) analysis of verbal behavior. The VB-MAPP is the product of collaboration and 30 years of field-testing and revisions. It has sold an estimated 125,000 copies in over 25 different 
countries. The VB-MAPP is it is made up of five components that assess and track verbal and related skills. There are two components that are particularly important; the VBMAPP Skills Assessment and the Task Analysis and Skills Tracking System.

The Skills Assessment contains 170 milestones that are used to evaluate a child's current verbal repertoire and other related skills. These milestones are sequenced and balanced across 3 developmental levels (0-18 months, 18-30 months, and 30-48 months). The Task Analysis and Skills Tracking System provides a communication and learning skills curriculum guide. The Task Analysis and Skills Tracking System component of the VB-MAPP contains the milestone from the Skills Assessment component but also provides a breakdown of the skills and other supporting milestones. Collectively they provide clinicians with a baseline level of performance, a direction for treatment goals, and a way to track skill mastery. However, the sequence of tasks that make up the curriculum have never been directly tested or evaluated. Thus, it is unknown whether the sequence of imitation skills in this curriculum guide accurately depicts the complexity of imitation tasks.

Espanola (2014) developed the Motor and Vocal Imitation Assessment in order to provide a empirically validated hierarchy of imitation skills that could serve as a protocol for selecting targets for imitation during intervention. The MVIA provides a baseline level of imitation skills, a validated sequence of imitation skills and a method of tracking skill mastery. The MVIA contains a comprehensive compilation of features and characteristics that determine the level of complexity of imitation tasks. The compilation of features and characteristics in the MVIA is the product of previous research examining the development of imitation (Jones, 2007; Rogers et al., 2003; Stone et al., 1997). 
The sequence of imitation tasks in the MVIA was evaluated by assessing imitation in 30 TD children and 30 children with ASD ranging from 14 to 39 months of age. Generally, TD children and children with ASD had similar patterns of imitation. Results showed children's performance varied depending on the type of imitation. For instance, children performed better on object imitation, followed by body imitation, vocal imitation, and facial imitation. Additionally, certain features of the task itself influenced performance. For example, object imitation that contained tasks characterized as meaningful yielded higher imitation scores than object imitation tasks characterized as not meaningful. The results provided an empirically validated hierarchy for imitation skills. The MVIA had high strong consistency score and high inter-rater reliability.

The MVIA and VB-MAPP are similar in that they both provide a protocol for selecting imitation skills for intervention. These protocols are different in the way that imitation is organized. For instance, the MVIA sequence begins with object imitation, is followed by body imitation, vocal imitation and ends with facial imitation. Whereas, the VB-MAPP sequence begins with facial imitation, then proceeds through body and object imitation and separately organizes vocal imitation. The sequence of imitation skills in the MVIA was empirically validated, as previously described, but the sequence of imitation skills in the VB-MAPP is not. Therefore, the MVIA may provide a better protocol than the VB-MAPP for selecting imitation skills for intervention. Improving the way that targets are selected for intervention may result in fast and efficient skill acquisition. The subsequent section contains a detailed review of the features and characteristics of the imitation tasks included in the assessment. 


\section{Hierarchy of Imitation Tasks.}

Imitation can be partly understood by investigating the features and characteristics of the action that is modeled. Piaget (1951) and Guillaume (1971) provided copious observations and hypotheses that have helped researchers identify and define these feature and characteristics.

Meaningful vs. Non-meaningful. The extent to which an action is considered meaningful or not, refers to whether the action entails common actions or the conventional use of items (Williams et al., 2004). Researchers have found that meaningful actions (e.g., putting hat on head) are more likely to be imitated than nonmeaningful actions (e.g., putting plate on head; Rogers, Bennetto, McEvoy, \& Pennington, 1996; Stone et al., 1997). Meaningful actions may be easier to imitate than non-meaningful actions because they are familiar and allow children to access previous information about the modeled response. Conversely, non-meaningful actions are easier to imitation than meaningful actions because children must depend purely on what was observed (Vanvuchelen, Roeyers, \& De Weerdt, 2007).

Visible (to self) vs. Not-visible (to self). The visibility of a modeled action denotes the degree to which individuals can see themselves performing the action (Jones, 2007; Abravanel, Levan-Goldschmidt, \& Stevenson, 1976). Studies demonstrate that actions are that visible (to self) are more likely to be imitated than actions that are not visible (to self; Jones, 2007). For instance, opening and closing hands would be easier to imitate than touching ears. According to Piaget (1951) and subsequent researchers, observing one-self affords visual cues that aid in accurate matching responses (Ray \& Heyes, 2011). When these visual cues are not available children must reproduce 
responses by simply relying on their knowledge about their own body and the body parts that are required to reproduce the response (Jones, 2007).

Sound vs. No-sound. The sound versus no sound distinction denotes whether or not an action results in auditory stimulus (Jones, 2007). An action that results in an auditory stimulus is easier to imitate than an action that does not (Abravanel et al., 1976; Dawson \& Adams, 1984). For example, shaking a maraca is easier to imitate than hugging a doll. Piaget (1951) hypothesized that sound becomes a learned cue for matching responses. That is, an accurate match would result in an auditory confirmation. Theorists continue to uphold this original hypothesis. The current consensus states that children use knowledge and feedback from their environment as the foundation for the expansion and acquisition of imitation (Heyes, 2010). Changes in a child's imitative repertoire are largely a result of advances in a child's comprehension of their own body parts, what they can do with those body parts and their social understanding and motivation (Jones, 2007).

The relative saliency of these features has never been directly investigated or explored. Therefore, it is difficult to understand if any one feature exerts more or less influence on the probability of imitation. Previous research has demonstrated that differences in performance may be more evident across some combination of features but not others (Espanola, 2014). For example, when object imitation was assessed using the MVIA, children scored higher on actions on objects that produced a sound and were meaningful versus actions on objects that did not produce a sound and were not meaningful. Yet, this was not true for actions on objects that did not produce a sound and were meaningful versus action that were not meaningful (Espanola, 2014). Simply stated, 
when the task did not produce a sound children performed similarly regardless of whether the action on object was meaningful or not. This indicates that particular conditions may diminish the influence that some features exert on the likelihood of imitation. Understanding which features of imitation task make a it more or less complex is a critical component in selecting targets for imitation intervention. This understanding allows clinicians to choose targets for imitation intervention that are appropriate for the child's current skill level, which in turn may lead to better intervention outcomes.

\section{Purpose of the Current Study}

Imitation is fundamental in general cognitive development and has been correlated with positive intervention outcomes (Kasari et al., 2008). Therefore, imitation is often selected for intervention in children with ASD who exhibit poor imitation skills (National Research Council, 2001). However, research has demonstrated that clinicians are not selecting imitation targets using empirically validated protocols (Ledford \& Wolery, 2011). Published curricula, such as the VB-MAPP, have a protocol for selecting imitation tasks for intervention, but these protocols have never been empirically validated. Thus, it is difficult to understand if the protocols for selecting imitation targets in these published curricula are appropriately detecting levels of skill complexity and accurately reflecting a sequence of imitation skills. The MVIA provides an empirically validated sequence of imitation tasks, ordered in increasing complexity, for the purpose of selecting appropriate targets for intervention.

The present study evaluated the use of two protocols for target selection during imitation intervention for children with ASD. Children were randomly assigned to two treatment groups; the groups differed in the sequence in which targets for intervention 
were selected. Targets for one group were selected on the basis of the VB-MAPP curriculum guide and targets for the other group were selected on the basis MVIA empirically validated instructional sequence organized from simple to complex. There are some distinct differences in how tasks are organized in the two protocols. The VB-MAPP separates echoic behavior from motor imitation, while the MVIA places echoic behavior at the end of the instructional sequence. Thus, children in the VB-MAPP comparison group work on both echoic and motor behaviors simultaneously, while children in the MVIA treatment group do not. The MVIA places vocal imitation between body imitation and facial imitation, suggesting that vocal imitation is more complex than body imitation but less complex than facial imitation. The other distinction lies in the order of motor imitation. Motor imitation in the MVIA begins with object imitation, then proceeds to body imitation and ends with facial imitation. On the other hand, the VB-MAPP begins with facial imitation, proceeds to body imitation, and then moves to object imitation.

These differences in the sequence of target selection may greatly influence how quickly children acquire imitation skills. If targets are selected that are not yet appropriate for the child's current level, then skill acquisition may take longer. Moreover, because of high levels of complexity some skills may never reach criteria for mastery. Conversely, if skills are targeted in a sequence ordered fro simple to complex time and resources may be better be allocated to more attainable and socially significant targets. In general, appropriate procedures for target selection are a critical component of a successful and effective intervention. 


\section{Hypotheses}

The following predictions were made regarding the performance on imitation tasks in this sample:

Hypotheses 1: Object, body, facial and vocal imitation scales from the Motor and Vocal Imitation Assessment a will be positively correlated and have a high measure of internal consistency.

Hypotheses 2: Participants in both groups will demonstrate an increase in their ability to imitate after the onset of treatment, as determined by skills that reach the mastery criteria. Hypotheses 3: Participants in the MVIA treatment group will demonstrate a higher number of skills mastered than participants in the VB-MAPP comparison group.

Hypotheses 4: Participants in the MVIA treatment group will acquire skills more efficiently than the participants in the VB-MAPP comparison group, as determined by the ratio of trials to mastery and total trials.

Hypotheses 5: Participants in the MVIA treatment group will spend less time on skills that never reach mastery, as determined by the number of trials of skills that never reach mastery.

Hypotheses 6: Participants in the MVIA treatment group will demonstrate higher levels of responding in general, as determined by the sum of full and partial imitation. Hypotheses 7: Imitation performance, as measured by the Motor and Vocal Imitation Assessment will significantly predict autism severity, as measured by the Autism Diagnostic Observation Schedule (ADOS) and the Social Responsiveness Scale (SRS). Hypotheses 8: Imitation performance, as measured by the Motor and Vocal Imitation Assessment will significantly predict language, as measured by the receptive and 
expressive scores in the Mullen Scales of Early Learning (MSEL) and the MacArthur Communicative Development Inventory (MCDI).

\section{Significance of Current Study}

Core skill deficits are amongst the first set of behaviors taught to children with ASD in EIBI programs (Kasari et al., 2008). Imitation is a core skill that is often chosen for treatment because of its correlation with the development of general cognitive abilities, communication, and social skills (Charman et al., 2003; De Giacomo et al., 2009; Miniscalco et al., 2014; Stone \& Yoder, 2001; Toth et al., 2006). Imitation is also commonly targeted for intervention because it can be used as a tool to teach other behaviors. This is particularly true for interventions that target the acquisition of verbal communication skills, as treatment packages in many of the early intervention manuals (e.g., Leaf \& McEachin, 1999; Lovaas, 2003) often rely on the assumption that children have vocal imitation or echoic repertoires when teaching expressive communication skills. Therefore, difficulties in vocal imitation also result in difficulties in teaching children to verbally communicate. Overall, the role that imitation plays in the acquisition of some of these critical skills and its usefulness as a training tool emphasizes the need to target imitation deficits in children with ASD during early intervention.

Studies evaluating the effectiveness of interventions targeting imitation skills have focused on comparing different modalities of treatment (e.g., reciprocal imitation training versus. video modeling; Cardon \& Wilcox, 2011) or have provided a review of effective treatment procedure (e.g., best prompting strategies for imitation intervention; Ledford \& Wolery, 2011). However, researchers have not particularly evaluated how the selection of targets for intervention can effect skill acquisition. 
The present study is the only study, to date, that evaluated the characteristics of the behavior being targeted for imitation intervention. Evaluating the effects of different sequences of target selection may provide critical information regarding the development of a successful intervention. Researchers and clinicians could use this information create interventions that are more appropriate for the child, more effective at bringing about behavior change and most likely going to result in the best use of time and resources. In sum, identifying appropriate targets may ensure that there is a steady acquisition of skills and that the skills being targeted are appropriate for the child's current skills level.

\section{Measures}

Social Responsiveness Scale (SRS; Constantino, 2002) The SRS is a quantitative scale that contains a total of 65 items. These items measure the severity and type of social impairment in children with ASD. The measure takes approximately 15-20 minutes to complete. The SRS is comprised of items that determine social awareness, social information processing capacity for reciprocal social responses, social anxiety/avoidance, and characteristic autistic preoccupations. The SRS provides a summary scale score; this score denotes an index of severity of social deficits associated with ASD symptomology. High scores describe a greater degree of severity of social impairment. The SRS was used to confirm ASD diagnosis; a cutoff score of 75 was used on the basis of current recommendations (Schanding Jr., Nowel, Goin-Kochel, \& Williams, 2012).

Mullen Scales of Early Learning (MSEL; Mullen, 1995). The MSEL provides a measure of intellectual development and readiness for school. The measure is for children from birth to 68 months. The MSEL raw score for cognitive and motor ability uses five scales: Gross Motor, Visual Reception, Fine Motor, Expressive Language, and Receptive 
Language. The raw score for these five scales provide a T-score, a percentile ranks, an age equivalent score, and an overall early learning composite score.

Autism Diagnostic Observation Schedule-Generic (ADOS; Lord et al., 1994). The ADOS is often used to assess and diagnose ASD. The standardized behavioral observation assesses ASD symptoms across a variety of ages, developmental levels, and language skills. The semi-structured assessment is made up of a variety of play-based situations that are meant to evoke social and communicative interaction with the examiner. These observations produce a score on 25 items across four domains: social interaction, communication, repetitive and stereotyped behaviors, and play. A summary score is calculated using select items within each domain. This summary score provides a cutoff an ASD diagnosis. This assessment was conducted by an examiner that met the reliability criteria of greater than $80 \%$ exact agreement in scoring and administration. In order to participate in this study all children had to meet the cutoff score for an ASD diagnosis.

MacArthur Communicative Development Inventory (CDI; Fenson et al., 1993). The MCDI is a parent rating that systematically assesses caregiver report of their child's language. The questionnaire focuses on comprehension, nonverbal gestures, early vocabulary and the early stages of grammar. Caregiver's report of total raw word production score was used; this score is made up of the number of words endorsed by the caregiver across 22 different categories (e.g., clothing, foods, action words).

Verbal Behavior Milestones Assessment and Placement Program (VB-MAPP; Sundberg, M. L., 2008). The curriculum breaks imitation down into two main branches echoic (vocal imitation) and motor imitation (which includes facial imitation). Echoic is a 
type of verbal behavior in which the response shares point-to-point correspondence and formal similarity with the controlling auditory stimulus that precedes it (Skinner, 1957). Point-to-point correspondence is present when all the components in the controlling stimulus (beginning, middle, and end) corresponds or matches all the components of the response. Formal similarity occurs when the responses both have the same mode (e.g., visual, tactile, auditory). For instance, a child saying "ball” as a result of hearing a peer say "ball" is engaging in echoic behavior.

The VB-MAPP assesses echoic behaviors using the Early Echoic Skills Assessment (EESA). The EESA groups words into five sub-sections ordered in increasing complexity: a) simple and reduplicated syllables (e.g., /ah/, /wow/, /bye//bye/); b) two syllable combinations (e.g., /icky/, /baby/, /open/); c) three syllable (e.g., /UH/-/oh/, /no/-/WAY/, /MY/-/mommy/); and d) prosody in other context (e.g., echoes whispering). Echoic behavior does not have a Task Analysis and Skills Tracking System component. Thus, the EESA was used to guide the direction of treatment.

The Task Analysis and Skills Tracking System for motor imitation under level one was used to guide the direction of treatment for motor imitation. Level 1 (0-18 months) contains five major developmental milestones. These milestone include: a) imitates two gross motor movements; b) imitates four gross mother movements; c) imitate eight gross motor movements, two of which include objects; d) spontaneously imitates motor behavior; and e) imitates 20 motor movements of any type. These developmental milestones are further broken down into individual skills. Additionally, skills are ordered in a increasing complexity. For instance, imitating mouthing 
movements (skill 1-a) precedes imitating 2 actions with objects (skill 2-a). See figure 1 for a general sequence of skills in the VB-MAPP.

Motor and Vocal Imitation Assessment (MVIA; Espanola, 2014). The MVIA assesses behaviors (vocal imitation) and motor imitation. The assessment was developed by the author and was created for the purpose of providing an assessment tool for imitation and an empirically validated protocol for target selection during imitation intervention. In order to begin intervention all participants needed to demonstrate low levels of pretreatment imitation as indicated by lower than $50 \%$ (32 points) on this assessment.

The assessment contains 32 one-step imitation tasks; eight object imitation, eight body imitation, eight facial imitation, and eight vocal imitation tasks. Tasks within object imitation are produced by crossing two features: (a) meaningful and non-meaningful actions and (b) actions that produce sound and actions that do not produce sound. Similarly, tasks within body imitation were produced by crossing two features: (a) visible (to self) and not-visible (to self) and (b) actions that produce sound and actions that do not produce sound.

Facial imitation tasks are comprised of any movements that required the use of oral-facial muscles (i.e., opening mouth, tongue protrusions, and pursing lip). Facial imitation tasks are organized into four groups and each group contained two tasks. Vocal imitation is organized in the same way. The tasks are organized into four groups: vocal play, canonical babbling, non-reduplicated speech and common words. Vocal play entails tasks that had consonant-like and vowel-like sounds (i.e., /b/, /d/, /m/, /u/ etc.). Canonical babbling entails tasks that had reduplicated true syllables (i.e., /da/-/da/, /ba/- 
/ba/). Non-reduplicated speech entails tasks that had consonant and vowel sounds that were not repeated (i.e., /da/-/di/, /la/-/da/).

Participants were encouraged to explore the objects in the assessment and develop a comfortable relationship with the examiner during a 10-minute free play condition. The tasks were presented using a playful and game-like approach. The researcher first gains the learners attention by calling his/her name. Then, the instruction "do this" or "say" is presented. This instruction is followed by the modeled action. For imitation tasks that involved the use of objects, the object was first placed in front of the participant. The participant was exposed to the object for approximately 10s and the instruction was only delivered if the participant was not currently engaging in the target response. This positively confirmed that any matching response after the modeled action was true imitation. If during the exposure, the participant performed the task, the researcher moved to another task in the assessment. Verbal descriptions of the actions were not provided. If the child did not engage in the target action within 5s, the task was finished. Praise and other social stimuli (e.g., tickles, high five) were provided non-contingently. The researcher did not label the responses. If the child did not respond within $5 \mathrm{~s}$, the task ended. Praise and social stimuli (e.g., tickles, high five) were non-contingently provided during the assessment.

Every participant was exposed to each task a total of three times. The tasks were assessed in random order. This safeguard is implemented in order to avoid fatigue affecting the participant's performance. The scoring system is a modified version of the Motor Imitation Scale scoring system (Stone et al., 1997). Response accuracy was scored on a three-point scale: successful imitation was scored as two points (i.e., touch nose), 
partial imitation was scored as one point (i.e., touch mouth instead of nose) and failure to imitate or non-responding was scored as zero points. Partial imitation was scored under the circumstance that the participant makes an attempt to imitate but was missing one or more components the modeled task. Failure to imitate or non-responding was scored when the participant either performs a different behavior or does not respond at all. The sum of the best score from the three trials yielded the total imitation score.

Preference Assessment. As previously discussed, reinforcers are stimuli that when presented increase the future probability of a response. Reinforcers are regularly used during intervention to teach children new response. It can be particularly difficult to find stimuli that function as reinforcers because in most cases, children cannot communicate their preferences (Carr, Nicolson, \& Higbee, 2000). Highly preferred stimuli were identified via a brief informal preference assessment. The preferrence assessment was conducted at the beginning of each session, and as needed, during the session (Davis, Dacus, Strickland, Machalicek, \& Coviello, 2013). This preference assessment entails providing the child with an array of 2-5 items hypothesized to be highly preferred and encouraging the child to reach for preferred item.

Preference was assessed by observing behaviors such as: (a) approaching or rejecting the items, (b) the frequency with which the child reached or interacted with the item, and/or (c) the duration the child spent engaged with the item (Logan et al., 2001; Pace et al., 1985). Approaching items, high frequency of interaction and a long duration of engagement with the items implied that the item was highly preferred. Items that were chosen during the preference assessment were delivered contingent upon the target 
response. If no items were chosen, then a new preference assessment was conducted with a variety of new items.

\section{Methods}

\section{Study 1}

The purpose of study one was to determine the effect of target selection for imitation skill on intervention outcomes in children with ASD. Particular attention was placed on skill mastery, efficient use of time and active responding.

\section{Participants}

Participants from this study were recruited from the EIBI and the Summer Treatment Program at the Center for Children and Families in Florida International University (FIU). These programs offer individualized behavior analytic intervention and focus on the development of core socio-communicative and adaptive skills.

All participants met the following inclusion criteria: (a) all participants had a diagnosis of ASD that was made by a licensed psychologist; (b) all diagnosis were further confirmed using the cutoff criteria for the Autism Diagnostic Observation Schedule (ADOS; Lord, Rutter, DiLavore, \& Risi, 1999) and the Social Responsiveness Scale (SRS; Constantino, 2002); (c) none of the participants had any diagnosed genetic or neurological disorder (e.g., seizures); (d) at the onset of the study participants had a chronological age between 24 and 56 months; (e) all participants had low levels of pretreatment imitation, as determined by a score at or below $50 \%$ on the MVIA.

A total of 25 participants were recruited. Five participants were excluded from the analysis: two of these participants did not meet the cutoff criteria for low levels of pre-treatment imitation, the other two participants completed less than $50 \%$ of the 
treatment sessions and the last participant was excluded as a result of procedural error.

The final sample consisted of 20 randomly assigned participants. Participants were either assigned into a comparison group following the curriculum guide in the VB-MAPP or a treatment group following the instructional sequence in the MVIA protocol.

Participants in the comparison and treatment group did not differ on measures of mental age. Measures of mental age (MA) were assessed by the Mullen Scales of Eearly Leanring (MSEL), $t(18)=1.4, p=.619$. MA was calculated by multiplying the MSEL composite score and the CA, then dividing the product by 100 (as seen in Young et al., 2001). The MVIA treatment group had a mean CA of 37.3 months and a mean MA of 18.23 months. The VB-MAPP comparison group had a mean CA of 35.83 months and a mean MA of 17.5.

Table 1

Study 1: Summary of Sample Characteristics

\begin{tabular}{lccccccccc}
\hline & \multicolumn{1}{c}{ Group } \\
\cline { 2 - 11 } & \multicolumn{1}{l}{ MVIA Treatment Group } & $\begin{array}{c}\text { VBMAPP Comparison } \\
\text { Group }\end{array}$ & \multicolumn{3}{c}{$\begin{array}{c}\text { Inclusion Criteria Not } \\
\text { Met }\end{array}$} \\
\hline & $n$ & $M$ & $S D$ & $n$ & $M$ & $S D$ & $n$ & $M$ & $S D$ \\
CA & 9 & 37.3 & 8.02 & 11 & 35.83 & 7.46 & 5 & 32.6 & 7.3 \\
Mental Age & 9 & 18.23 & 3.93 & 11 & 17.5 & 3.65 & 5 & 18.48 & 2.26 \\
MSEL Composite Score & 9 & 49 & 0 & 11 & 49.55 & 1.8 & 5 & 58.2 & 10.84 \\
MCDI (number of words) & 9 & 83.67 & 65.54 & 10 & 25.9 & 15.06 & 5 & 84.4 & 79.73 \\
ADOS cutoff score & 9 & 18.78 & 3.15 & 11 & 20 & 4.33 & 4 & 15.75 & 2.75 \\
SRS total score & 9 & 93.67 & 33.3 & 11 & 87.82 & 33.14 & 5 & 93.4 & 30.98 \\
\hline
\end{tabular}

Note. Sample sizes vary due to missing data; $n=$ sample size; $M=$ Mean; $S D=$ Standard Deviation; $\mathrm{CA}=$ Chronological Age; Mullen Scales of Early Leaming; MCDI=MacArthur Communicative Development Inventory; ADOS=Autism Diagnostic SRS=Social Responsiveness Scale

Participants in the comparison and treatment group did not differ in autism severity, as measured by the Autism Diagnostic Observation Schedule (ADOS), $t(18)=$ -.705, $p=.490$. The MVIA treatment group had a mean pre-treatment imitation score of 
18.78 and the VB-MAPP comparison group had a mean pre-treatment imitation score of 20. Table 1 depicts demographic and descriptive information, including gender, chronological age, mental age and summary scores from all the measures.

Participants in the comparison and treatment group did not differ in their pretreatment imitation, as measured by the MVIA, $t(18)=1.77, p=.094$. The MVIA treatment group had a mean pre-treatment imitation score of 12.4 and the VB-MAPP comparison group had a pre-treatment imitation score of 7.54. A breakdown of these score is discussed in further detail in the results section.

\section{Setting and Materials}

All sessions were conducted during the EIBI or Summer Treatment Program. Caregivers were informed about the present study and were provided with the consent form (see Appendix A). For children whose parents consented to being part of the study and met the inclusion criteria, the sessions were conducted in the classroom and were incorporated into their daily treatment sessions. For the duration of the study participants did not receive additional imitation or echoic training as part of their treatment programs at FIU.

A workstation was assigned for the study. The workstation had a small child sized table and chair along with a bookshelf. The workstation looked similar to the other workstations in the classroom. Age appropriate toys, highly preferred items and snacks were used throughout the intervention. A minimum of 33\% of sessions were recorded for independent coding of inter-observer agreement and treatment fidelity per participant. A variety of materials were used for the purpose of data collection (e.g., pen, paper, clip boards, etc.). 


\section{Procedures}

Children were randomly assigned into two different treatment conditions. Random assignment was be conducted on MS Excel using the function, $R A N D()$. The function yields a random five-digit number between 0 and 1 for every case. The procedure assigned participants to a condition on the basis of chance; each participant had a nonzero probability of being assigned to a condition. Odd numbers were assigned to the MVIA treatment group and even numbers were assigned to the VB-MAPP comparison group. The intervention procedures for the MVIA treatment group and the VB-MAPP comparison group were identical. The only difference between the two groups was the sequence of imitation targets. Random assignment made the two groups randomly similar to each other, so that the groups were equivalent at the onset of the study. This reduced the probability that chance alone could result in any observed differences.

On average there were two to four sessions per day, each session consisted of 5 trials with a total of 20 trials per day. The study consisted of an average of 70 sessions, approximately 15 minutes in length. The therapist and research assistants were blind to the hypotheses. Treatment procedures for the present study were the same as the treatment procedures used in the classroom. Thus, the child's trained therapist was able to conduct all sessions. A research assistant was present to record treatment fidelity and probe for generalization.

Generalization probes consisted of imitation tasks varying across different categories and features. The probes were assessed at various time points throughout the 
intervention sessions. The generalizations probes were used to assess the generalizability of imitation outcomes (See Appendix B for Generalization Probes).

Selecting Targets for Intervention. The only controlled difference between the treatment groups was the protocol used for selecting target behaviors. The sequence of targets selected for the MVIA group is derived on a hierarchy of imitation skills. Imitation in the MVIA is broken down into four categories: object, body, vocal, and facial imitation. Each type of imitation is broken down into four components, each characterized by different features of imitation (e.g., visible-to-self vs. not visible-toself). The protocol is organized on a continuum of difficulty, with targets later in the hierarchy reflecting more complex skills. Every component has a set criterion for mastery. This criterion has to be met before the participant can transition to the targets in the next component (See Appendix C for MVIA Criteria for Goal Mastery).

Similar to the MVIA treatment group, imitation skills in the VB-MAPP are ordered sequentially, in increasing complexity (See Appendix C \& D for VB-MAPP Criteria for Goal Mastery). Once again, it is important to reiterate that the sequence of the tasks in the MVIA protocol have been empirically validated but the sequence of the tasks in the VB-MAPP protocol have never been directly tested and are not emperically validated. There are some distinct differences in how imitation skills are ordered. Overall, the VB-MAPP contains the following sequence of imitation, ordered in increasing complexity: facial, body, and object imitation. Vocal imitation is not included in this sequence and is separated into it's own category within the same developmental age level. Thus, it was targeted simultaneously with motor intervention. 
The MVIA diverges from this sequence, suggesting that imitation should be targeted based on the following sequence ordered in increasing complexity: object, body, vocal, and facial imitation. The sequences in the two protocols are notably incongruous. The differences between the two sequences of imitation are depicted in figure 1.

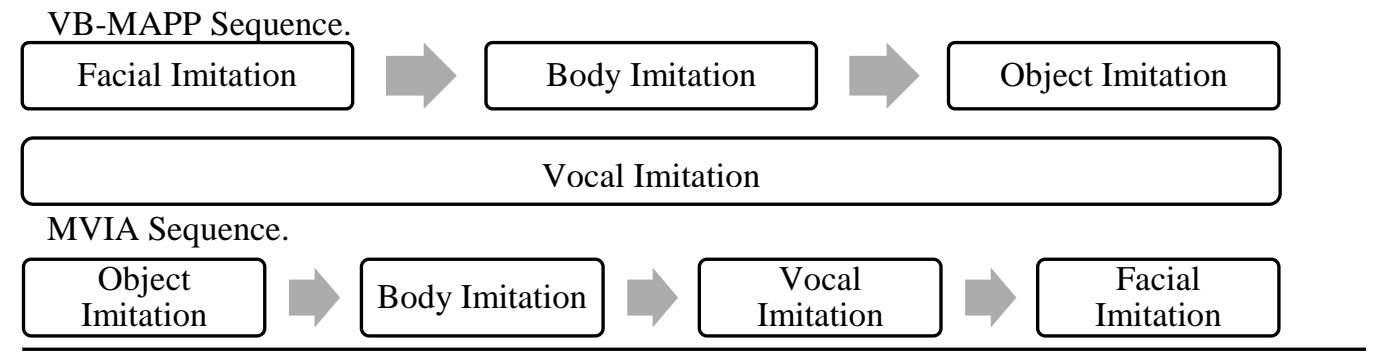

Figure 1. Sequence of imitation skills for the VB-MAPP and the MVIA.

A research assistant blind to the study's hypotheses selected targets in accordance with the procedures for each protocol. The investigator reviewed target selection in order to ensure that the research assistant was selecting targets according to the specified procedures. However, the investigator did not select or change any targets for either group. Errors in target selection resulted in a correction procedure, which entailed directing the research assistant to the appropriate protocol for modifications.

Baseline. An AB Design was used for all imitation targets. The two-phase design consists of a no-intervention baseline phase (A) and an intervention phase (B). The A phase measures the natural frequency of the target behavior, while the B phase measures changes associated with the implementation of the intervention (Johnston \& Pennypacker, 2009). The strategy involves repeatedly exposing a participant to a specific condition (e.g., baseline), while trying to reduce or eliminate any extraneous influences on the response, and reaching a stable pattern of behavior before introducing the next condition (e.g., intervention). Steady state responding is observed when there is an 
absence of: variability or an increasing/decreasing trend (Johnston \& Pennypacker, 2009).

The intervention was characterized as adult directed discrete trial training. Baseline was introduced for a minimum of 2 trial blocks (10 trials). Baseline trials were conducted in the same manner as the intervention trials but did not include prompting or reinforcement. Baseline trials consisted of a therapist sitting across from the child, presenting the $S^{\mathrm{D}}$ "do this," or "say” and modeling the target behavior. If an object was being used the therapist handed the object to the child after they had modeled the target behavior. The therapist then recorded the response and moved on to the next trial. After a baseline trial a child would not receive access to highly preferred items, therefore, baselines trials were interspersed amongst easy tasks (e.g. "give me a high 5). This allowed the child to gain access to preferred items during baseline sessions. This helped prevent the occurrence of any problem behaviors and maintained behavior momentum during baseline sessions. Responses were recorded as follow: successful imitation was scored as 2 points (i.e., touch nose), partial imitation was scored as 1 point (i.e., touch mouth instead of nose) and failure to imitate or non-responding was scored as 0 points.

Intervention. Intervention procedures were the same as the baseline procedures with the addition of prompting and the delivery of highly preferred items contingent upon appropriate responding. A progressive time delay most-to-least prompting procedure was used during intervention. The progressive time delay most-to-least prompting procedure supports active responding from the participant because they are not waiting to receive help, as may occur with least-to-most prompting (Glendenning, Adams, \& Sternberg, 1983). Delivering a prompt before errors takes place during initial training, and 
progressively fading these prompts seems to result in the most efficient learning (Ledford \& Wolery, 2011; see Figure 2 for prompting hierarchy).

\begin{tabular}{|c|c|c|}
\hline Level 1 & $\begin{array}{l}\text { Full physical guidance (i.e. hand over hand) is immediately } \\
\text { used to help the learner engage in the response. If the } \\
\text { learner begins to engage any part of the response } \\
\text { independently the therapist moves down to level } 2 \text { (i.e. will } \\
\text { bang the hammer, but will not initiate the behavior). }\end{array}$ \\
\cline { 1 - 2 } Full physical guidance & $\begin{array}{l}\text { Partial physical guidance is immediately used to help the } \\
\text { learner engage in the response (i.e. the therapist touches the } \\
\text { learner hand with one finger to initiate response). If the } \\
\text { learner is able to engage in the entire response, but does not } \\
\text { do so consistently the therapist moves down to level 3. }\end{array}$ \\
\cline { 1 - 2 } Partial physical guidance & $\begin{array}{l}\text { Therapist provides the learner with 2-3 seconds to respond } \\
\text { and follows the learner's movement closely with his/her } \\
\text { hands without touching the learner, until prompting is no } \\
\text { longer necessary. }\end{array}$ \\
\hline Prompt delay 3 & \\
\hline \hline
\end{tabular}

Figure 2. Prompting hierarchy and procedures for moving through the hierarchy.

Highly preferred items were delivered contingent upon the correct response for both prompted and unprompted response. Under circumstances where the participant did not engage in the target behavior (e.g., did not match a vocal response) and the behavior could not be prompted any further, the therapist ended the trial and presented a series of easy unrelated tasks. Appropriate responding during these tasks resulted in access to mildly preferred items. This ensured that the participant had a chance to access preferred items during the session.

An error-correction procedure was implemented in order to help strengthen the association between the modeled action and the matching response (McGhan \& Lerman, 2013). Contingent on an error or no response, the therapist immediately re-presented the 
$S^{\mathrm{D}}$. Then, the least intrusive, but most effective prompt was delivered. If the prompt was not effective, the therapist moved through the prompting hierarchy to more intrusive prompts.

Reliability and Inter-observer Agreement. Inter-observer agreement (IOA) is described as the degree of agreement for an observed event between two independent observers (Cooper et al., 2007). Reliability and measurement validity in behavioral research is often assessed using IOA (Watkins \& Pacheco, 2000). High IOA increases the confidence that the definition of the target behavior is accurate and unambiguous, that the measurement system is clear and easy to use. This leads an increase in the believability of the data and the resulting interpretations. The current standards recommend that IOA should be $90 \%$ or greater for a measure that is well-established and $80 \%$ or greater for a new variable (Cooper et al., 2007).

Inter-observer agreement is often calculated in a variety of ways, but all measures of agreement rely on the following conditions to maintain validity of the measurement: a) observers must use the same definition for target behavior, same observation procedures, codes and measurement devices; b) observers must measure the same subjects for the same observation period, and (c) observers must be independent.

The present study had two independent observers collect data for a minimum of $33 \%$ of sessions across phases (baseline and intervention). Agreement was defined as a trial in which both observers recorded the same occurrence of behavior. Inter-observer agreement was calculated using trial-by-trial IOA, which is best used for trial based responding in which binary outcomes are measured (e.g., occurrence/non-occurrence, yes/no, on-task/off-task). For the purposes of calculating IOA, agreement was determined 
by the occurrence or non-occurrence of the target behavior. Results showed that IOA for the MVIA treatment group was 91.8\% (range: 85\% - 98\%) and for the VB-MAPP comparison group was $95.2 \%$ (range: $85 \%$ - 98\%).

Treatment Fidelity. Treatment fidelity (TF) describes the various methodological strategies conducted to assess and enhance the reliability and validity of behavioral interventions. These methodological practices are directed towards guaranteeing that studies reliably and validly test intervention procedures (Bellg et al., 2004). The main purpose of TF is to increase scientific confidence in the idea that the changes in the variable of interest are related to the independent variable (Borrelli et al., 2005). Internal and external validity are both enhanced by TF, high degree of TF denotes treatment that is both replicable and generalizable (Borrelli et al., 2005).

Treatment fidelity was evaluated for the purposes of assessing adherence to intervention procedures and the competency of therapists (See Appendix E for treatment fidelity data sheet). Treatment fidelity was calculated by measuring the number of correctly implemented trials and dividing them by the total number of trials and multiplying by 100 . The product was the percentage of correctly implemented trials. A task analysis of intervention components was employed in order to assess if each step of a trial was implemented appropriately or not. A trial was scored correct if the therapist completed all the steps in the research protocol. A trial was scored incorrect if the therapist missed or incorrectly performed one or more steps in the research protocol.

Treatment fidelity was recorded for an average of $80 \%$ of sessions across all phases, treatment conditions, and participants. The MVIA treatment group had an average treatment fidelity score of $96 \%$ and the VBMAPP comparison group had an 
average TF score of 97\%. The TF scores for the MVIA treatment group ranged from 97\% to $100 \%$ and the TF scores for the VB-MAPP comparison group ranged from 95\% to $99 \%$.

Intervention procedures in the present study were the same procedures used in the classroom wide intervention. Thus, therapists were already familiar with the protocol for implementing the imitation intervention. The therapist's experience implementing the intervention, the presence of the research assistant and feedback procedures may have in part attributed to high treatment fidelity.

At the end of the session the therapist was provided with two types of feedback on their performance: a) positive feedback for correctly following procedures and/or collecting data, and (b) corrective feedback for incorrectly following procedures and/or collecting data (DiGennaro, Martens, \& Kleinmann, 2007). Feedback was provided as per current recommendations for treatment integrity monitoring (Vollmer, Sloman, \& Pipkin, 2008). If any of the procedural guidelines were violated, the therapist was provided with immediate feedback and coaching at the end of the trial. Therapists received frequent positive feedback for correct implementation of procedures. Additionally, continued training and coaching sessions were implemented if at any time a therapist scored below $50 \%$ for any trial or lower than $80 \%$ for two consecutive days.

\section{Study 2}

The purpose of study two was to investigate the predictive nature of imitation skills. Recent studies suggest that imitation skills are correlated and in time can predict other central developmental areas also affected by autism (e.g., language) and core symptoms of autism. 


\section{Participants}

All participants recruited from study one, were included in study two. The final sample in study two included five participants that were excluded from the analysis in

Table 2

Study 2: Summary of Sample Characteristics

\begin{tabular}{lccccc}
\hline & \multicolumn{3}{c}{ Participants } \\
\hline CA & $n$ & $M$ & $S D$ & Min. & Mox. \\
Mental Age & 25 & 35.68 & 7.52 & 24 & 52 \\
MSEL Composite Score & 25 & 17.99 & 3.43 & 11.76 & 25.48 \\
MCDI (number of words) & 25 & 51.08 & 5.85 & 49 & 75 \\
ADOS cutoff score & 24 & 59.54 & 59.44 & 7 & 236 \\
SRS total score & 24 & 17.81 & 2.60 & 13 & 23 \\
MVIA & 25 & 91.04 & 31.56 & 28 & 145 \\
\hline
\end{tabular}

Note. Sample sizes vary due to missing data; $n=$ sample size; $M=$ Mean; $S D=$ Standard Deviation; $M i n=$ Minimum; $M a x=$ Maximum; CA=Chronological Age; Mullen Scales of Early Learning; MCDI=MacArthur Communicative Development Inventory; ADOS=Autism Diagnostic SRS=Social Responsiveness Scale; MVIA = Motor and Vocal Imitation Assessment

study one. Participants were between 24 and 52 months of age $(M=54.68, S D=7.51)$.

There were a total of 4 females and 21 males. The sample largely consisted of white Hispanics (96\%). A summary of the characteristics of the sample is depicted in Table 2.

\section{Results}

\section{Study 1}

Hypothesis 1: Object, body, facial and vocal imitation scales from the Motor and Vocal Imitation Assessment (MVIA) will be positively correlated and have a high measure of internal consistency.

A Pearson product-moment correlation coefficient was computed to assess the relationship between the four scales. There was a statistically significant positive 
correlation between all the variables (see Table 3). The internal consistency was also calculated; measures of internal consistency are displayed in four subscales (see Table 4). These scales were a function of form: Object (manual actions on objects), Body (actions without objects), Facial (actions using facial muscles), and Vocal imitation. Cronbach’s alpha was used to explore measures of internal consistency for all of the participants’ MVIA object, body, vocal and facial imitation summary scores and total imitation score (Cronbach, 1988). Internal consistency scores were as follows: MVIA total imitation score (.878), object (.708), body (.787), vocal (.867) and vocal (.659) imitation summary score. The high measures of internal consistency and the presence of positive correlations are consistent with previous findings (Espanola, 2014) and show that the tasks in the scales are in fact assessing the skills they were intended to measure.

Table 3

Correlations for Imitation Scales

\begin{tabular}{|c|c|c|c|c|}
\hline \multicolumn{5}{|c|}{ Imitation Scores } \\
\hline & OI & $\mathrm{BI}$ & FI & VI \\
\hline OI & - & & & \\
\hline BI & $.439 *$ & - & & \\
\hline VI & $400 .^{*}$ & $.524 * *$ & - & \\
\hline FI & $.461^{*}$ & $.573 * *$ & $.571 * *$ & - \\
\hline
\end{tabular}

Table 4 depicts pre-treatment imitation performance scores on the MVIA separated by group. As expected, imitation scores for participants that did not meet the inclusion criteria were higher than scores for participants in the study. This was due to the nature of the inclusion criteria, which excluded any participant scoring more than $50 \%$ (32 points) on the MVIA. As previously noted, the participants in the comparison and 
treatment group did differ in their measure of pre-treatment imitation, $t(18)=1.77, \mathrm{p}=$ .094 .

A detailed breakdown of the sum of imitation scores across all participants is represented in Figure 3. Imitation is ordered by category (e.g. object, body, vocal, facial imitation) and feature (e.g., meaningful vs. non-meaningful). Participants performed the best in object imitation, followed by body, facial and then vocal imitation. The graph also displays a hierarchy of imitation performance within each category. For instance, object imitation that was meaningful and made a noise had higher scores than those that did not make a noise. The graph shows that imitation performance for the participants in the present study followed the same sequence yielded in Espanola (2014). However, it is important to note that 9 of the 25 participants in the present sample were also in the

Table 4

Descriptive Statistics for the Motor and Vocal Imitation Assessment

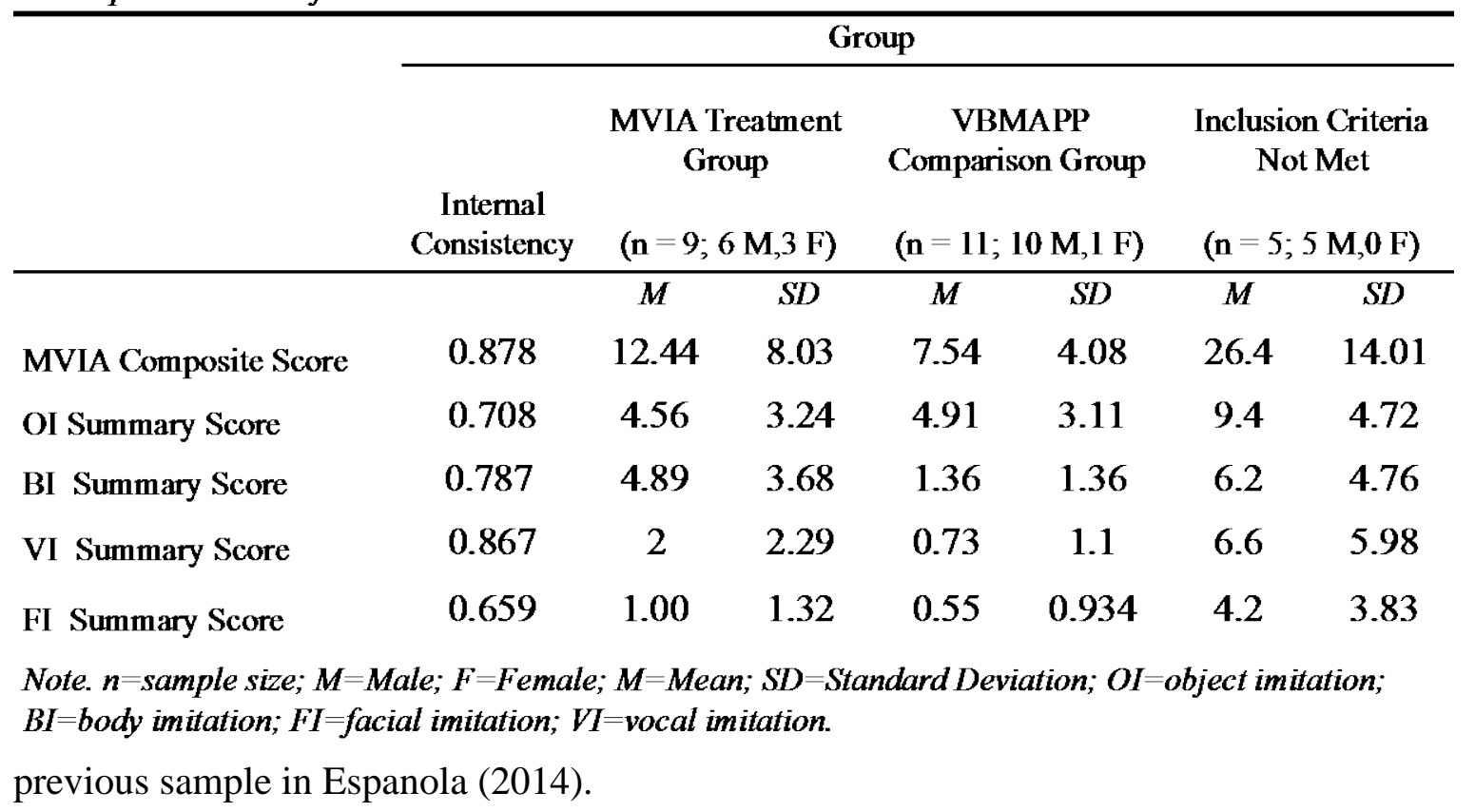




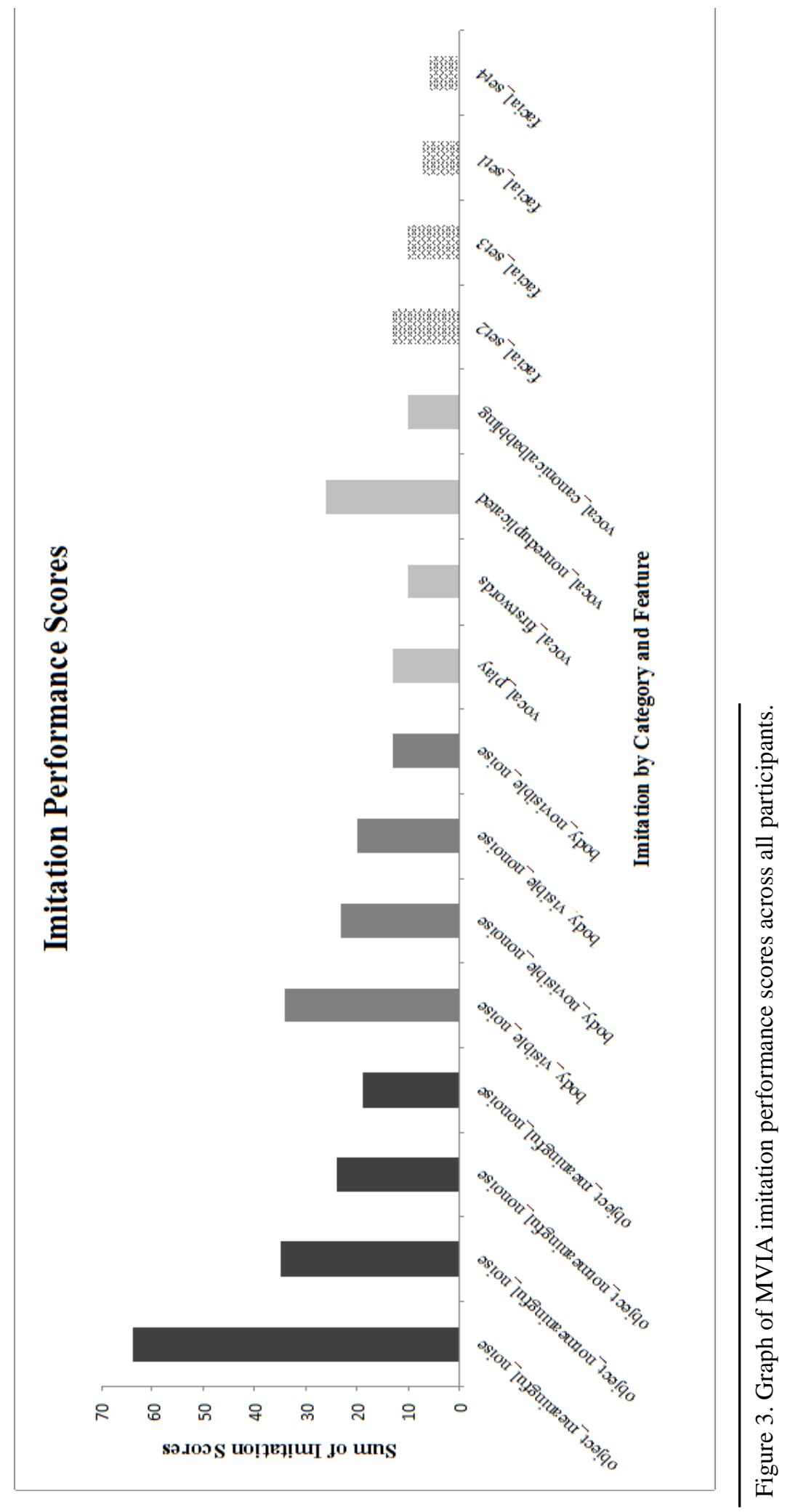




\section{Hypothesis 2: Participants in both groups will demonstrate an increase in their}

\section{ability to imitate after the onset of treatment, as determined by skills that reach the mastery criteria.}

Visual inspection is the principal method of analysis in behavioral research (Cooper et al., 2007). Visual inspection refers to the conclusions about the reliability or consistency of intervention effects via the visual examination of graphed data (Kazdin, 2013). Session percentages for each target during the baseline and intervention were graphically depicted for each participant. See appendix G-H for the graphed data of the last two participants from each group.

The baseline phase (depicted by phase A) provides a measure of the natural frequency of the targeted skill. The intervention phase (depicted by phase B) provides a measure of the changes related to the introduction of the intervention. For the purpose of moving efficiently through the hierarchy of imitation skills in both protocols, some targets were introduced simultaneously while other targets were staggered or introduced as other skills reached criteria for mastery.

Graphs depicting skills that were introduced simultaneously offer information regarding the child's current skill level and demonstrate that the child did not have the skill in their repertoire before the onset of the intervention. For skills that were introduced simultaneously, the data was evaluated and graphically depicted using AB design logic. However, this type of design is vulnerable to multiple threats to internal validity and cannot be used as conclusive evidence of experimental control. Thus, conclusions about the effectiveness of the intervention for both groups were further evaluated for those 
skills that were introduced as they reached criteria for mastery using multiple-baseline across behaviors design.

The experimental reasoning used for multiple-baseline designs is referred to as baseline logic, which contains three elements: prediction, verification and replication. Baseline logic assumes that changes in behavior after the introduction of the treatment phase are caused by the treatment rather than other extraneous variables. This design in particular tries to control for extraneous influences (e.g., maturation) by demonstrating that specific changes in a behavior are related to the introduction of the intervention at various points in time. Each instance in which the results are replicated attests to the generality of findings and also increases the trustworthiness in the reliability of the findings and subsequent interpretations.

\section{Hypothesis 3: Participants in the MVIA treatment group will demonstrate a higher number of skills mastered than participants in the VB-MAPP comparison group.}

Analysis of variance was used to examine group differences in imitation performance (see Table 5). Cohen's d effect size values were also calculated using Fvalue of the analysis of variance (Thalheimer \& Cook, 2002). Effect size values were used to determine the practical importance of any reported significant group differences. The results showed that the differences in average total skills mastered between the MVIA treatment group ( $n=9, M=7.44, S D=4.03)$, and the VB-MAPP Comparison Group ( $n=11, M=2.45, S D=4.13$ ) were statistically significant, $F(1,18)=7.37, p=$ $.014, \eta 2=.29$. These results show that the MVIA treatment group had a higher number of skills mastered than the participants in the VB-AMPP comparison group. The MVIA treatment group mastered an average of 7 behaviors, while the VB-MAPP comparison 
group mastered an average of 3 behaviors. Cohen's effect size was also very large ( $d=1.28$ ), which further underscored that these group differences were not only statistically significant but also of high practical importance.

All behaviors, across both groups were categorized as object, body, vocal and facial imitation for the purpose of further evaluating group performance. Skill acquisition as determined by total skills mastered per group demonstrated that skill acquisitions proceeded through the same hierarchy proposed in the MVIA protocol. For participants in both groups, object imitation had the highest number of skills mastered, followed by body imitation, then vocal imitation and facial imitation (See Table 6 for descriptive information for intervention outcomes).

It is important to reiterate that the participants in both groups were well matched in mental age, autism severity and pre-treatment imitation and that the only controlled difference between the two groups was the protocol used for target selection. Thus, any observed differenced between the two groups is most likely to be a result of differences in the protocol for target selection rather than extraneous sources.

Table 5

One Way ANOVA Results for Differences Between MVIA Treatment Group and the VBMAPP Comparison Group

\begin{tabular}{|c|c|c|c|c|c|c|}
\hline & \multicolumn{4}{|c|}{ Group } & & \multirow{3}{*}{ Effect Size } \\
\hline & \multicolumn{2}{|c|}{$\begin{array}{l}\text { MVIA Treatment Group } \\
\qquad(\mathrm{n}=9 ; 6 \mathrm{M}, 3 \mathrm{~F})\end{array}$} & \multicolumn{2}{|c|}{$\begin{array}{l}\text { VBMAPP Comparison Group } \\
(\mathrm{n}=11 ; 10 \mathrm{M}, 1 \mathrm{~F})\end{array}$} & & \\
\hline & $M$ & $S E$ & $M$ & $S E$ & $F$ & \\
\hline Total Tasks Mastered & 7.44 & 1.34 & 2.45 & 1.24 & $7.372^{*}$ & 1.28 \\
\hline $\begin{array}{l}\text { Proportion of Trial to } \\
\text { Mastery and Total Trials }\end{array}$ & 0.537 & 0.084 & 0.19 & 0.092 & $7.397^{*}$ & 1.28 \\
\hline $\begin{array}{l}\text { Trials that Never Reached } \\
\text { Mastery }\end{array}$ & 432.66 & 99.84 & 976 & 148.33 & $8.381^{*}$ & 1.37 \\
\hline Active Responding & 418.11 & 52.15 & 182.81 & 55.48 & $9.231^{*}$ & 1.43 \\
\hline
\end{tabular}


Hypothesis 4: Participants in the MVIA treatment group will acquire skills more efficiently than the participants in the VB-MAPP comparison group, as determined by the ratio of trials to mastery and total trials.

The analysis of variance also showed that the differences in the ratio of trials to mastery and total trials between the MVIA treatment group $(n=9, M=.537, S D=.252)$, and the VB-MAPP comparison Group ( $n=11, M=.190, S D=.306)$ were statistically significant, $F(1,18)=7.39, p=.014, \eta 2=.29$. These results show that the MVIA treatment group acquired skills more efficiently than the VB-AMPP comparison group. On average 53\% of trials resulted in skill acquisition in the MVIA treatment group while only $20 \%$ of trials resulted in skill acquisition in the VB-MAPP comparison group.

Cohen's effect size further demonstrated that the magnitude of the difference between the measures of efficient skill acquisition was large $(d=1.28)$.

Results from the breakdown of group differences by category show that for both groups the ratio of trials to mastery and total trials generally decreased as children moved through the sequence of skills; with object imitation showing the largest ratio and facial imitation showing the smallest ratio of trials to mastery and total trials.

Hypothesis 5: Participants in the MVIA treatment group will spend less time on skills that never reach mastery, as determined by the number of trials of skills that never reach mastery.

The MVIA treatment group $(n=9, M=432.66, S D=299.53)$ and the VB-MAPP comparison group ( $n=11, M=976.00, S D=491.98)$ were also statistically significantly different in the amount of trials that never reached mastery, $F(1,18)=8.38, p=.010, \eta 2$ $=.30$. These results demonstrate that the MVIA treatment group spent less time than the 
VB-MAPP comparison group on skills that never reached mastery. In relation to the MVIA treatment group, the VB-MAPP comparison group had nearly more than double the amount of trials on skills that never reached mastery. Cohen's effect size further established that the magnitude of the difference between the trials that never reached mastery was large $(d=1.37)$.

\section{Hypothesis 6: Participants in the MVIA treatment group will demonstrate higher levels of responding in general, as determined by the sum of full and partial imitation.}

Results sowed that the differences in the sum of full and partial imitation between the MVIA treatment group $(n=9, M=418.11, S D=156.45)$, and the VB-MAPP comparison group ( $n=11, M=182.81, S D=184.00$ ) were statistically significant, $F(1,18)=9.23, p=.007, \eta 2=.33$. The MVIA treatment group demonstrated higher levels of responding than the VB-MAPP comparison group. Overall, participants in the MVIA treatment group demonstrated active responding for more than double the amount of trials than the VB-MAPP comparison group. Cohen's effect size showed that the differences in active responding between the groups were also meaningful $(d=1.43)$.

There did not appear to be any patterns of active responding across the different types of imitation for neither the MVIA treatment group nor the VBMAPP comparison group. However, it is important to note that across the different types of imitation, the MVIA treatment group had more active responding than the VB-MAPP comparison group. 
Generalization. Generalization was probed every week with the exception of the Table 6

Intervention Outcomes by Imitation Type for MVIA Treatment Group and the VBMAPP Comparison Group

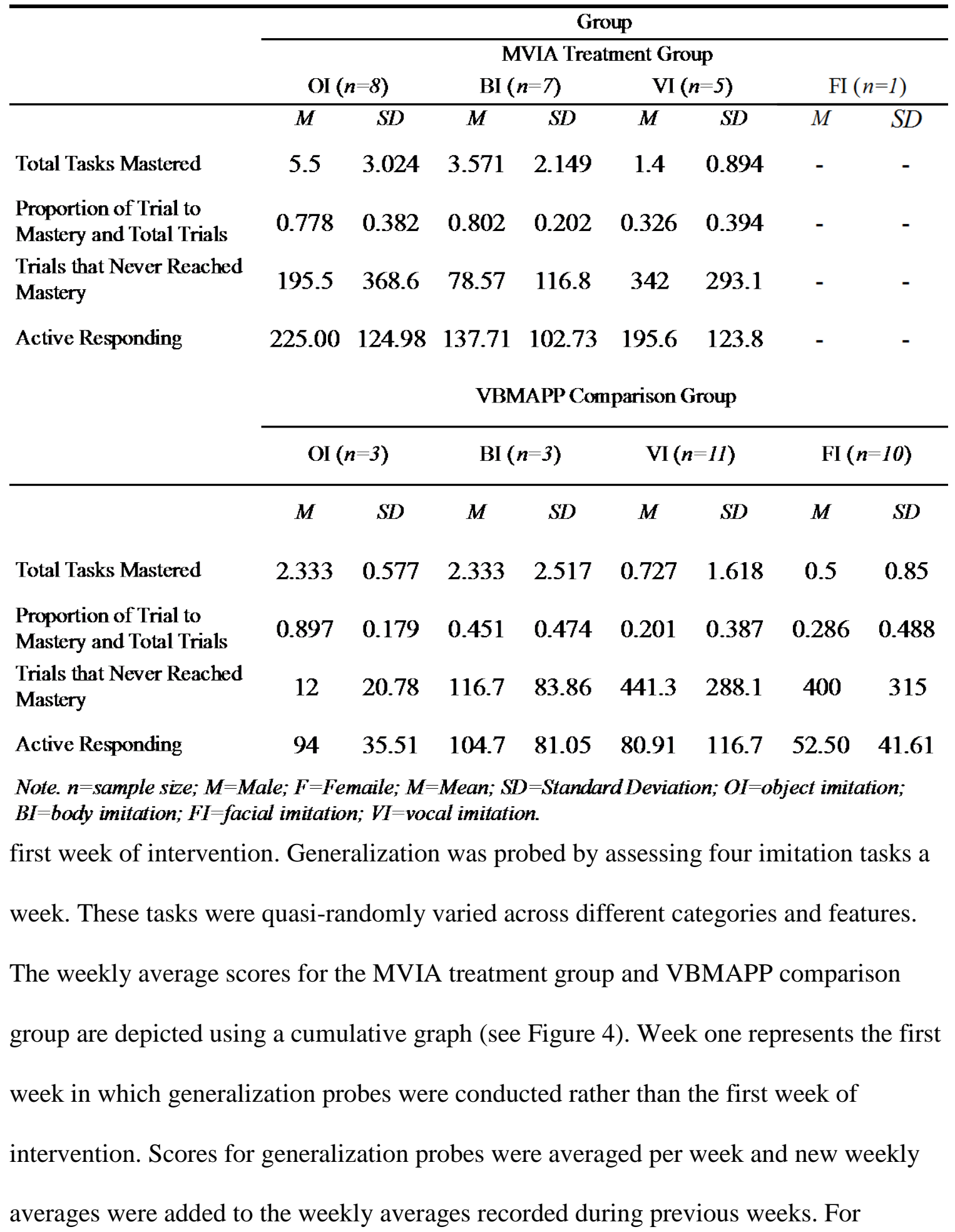


instance, week one the average generalization score was 4.2 for the MVIA treatment group, therefore the first data point was plotted at 4.2. During week two the average generalization score was 8.1 for the MVIA treatment group, therefore the next data point was plotted at 12.3. For cumulative records such as these, the steeper the slope the more that generalization has occurred.

Overall, participants in the MVIA treatment group had data paths depicting steeper slopes than the participants in the VB-MAPP comparison group. Visual inspection of the cumulative graphs indicates that the MVIA treatment group showed a higher degree of skill generalization than participants in the VB-MAPP comparison group.

\section{Cumulative Graph Generalization Probes}

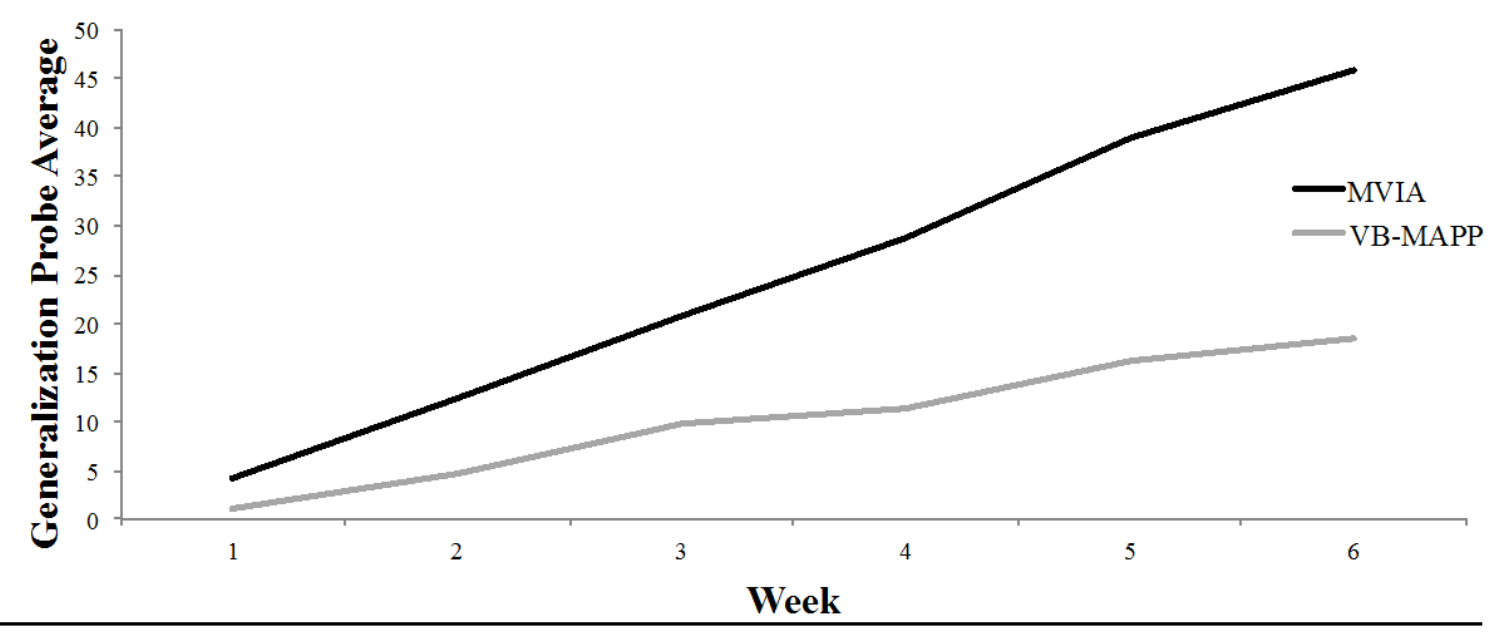

Figure 4. Cumulative graph representing generalization of imitation skills for the MVIA treatment group and the VB-MAPP comparison group.

\section{Study 2}


A correlation analysis was conducted in order to investigate the relationship between imitation and other developmental measures for children with ASD. Statistically significant correlations were further explored using linear regression. The ADOS and SRS scores were analyzed using their original metric; thus, the higher scores represent more severe symptomology, while for the other measures, higher scores represent superior adaptive behaviors. Largely, there were moderate to strong correlations between imitation and autism symptomology and imitation and expressive language. Table 7 shows the correlations amongst the analyzed variables.

Table 7

Correlations Between MVIA Scores and Developmental Measures for Children with ASD

\begin{tabular}{|c|c|c|c|c|c|}
\hline & \multicolumn{5}{|c|}{ Imitation Scales } \\
\hline & OI & BI & VI & FI & TI \\
\hline $\operatorname{ADOS} c s\left(\mathbb{N}^{a}\right)$ & $-.571^{* *}$ & -.143 & -.244 & -0.278 & $-.475^{*}$ \\
\hline MSEL:VP & -.196 & $.528 * *$ & .135 & .122 & .212 \\
\hline MSEL: FM & -.011 & -.232 & .260 & .256 & .264 \\
\hline MSEL: RL & -.106 & -.002 & .324 & $.586 * *$ & .237 \\
\hline MSEL:EL & .019 & .493 & $.598 * *$ & $.489 *$ & $.585^{* *}$ \\
\hline MSEL composite & .167 & .084 & .081 & $.555^{* *}$ & .275 \\
\hline $\operatorname{MCDI}\left(p w u / N^{a}\right)$ & .122 & $.484^{*}$ & .287 &.-108 & .352 \\
\hline $\operatorname{MCDI}\left(p w p / N^{a}\right)$ & -.033 & .066 & $.557^{* *}$ & $.769^{* *}$ & $.455^{*}$ \\
\hline SRS & -.016 & .357 & .158 & -.010 & .202 \\
\hline \multicolumn{6}{|c|}{ 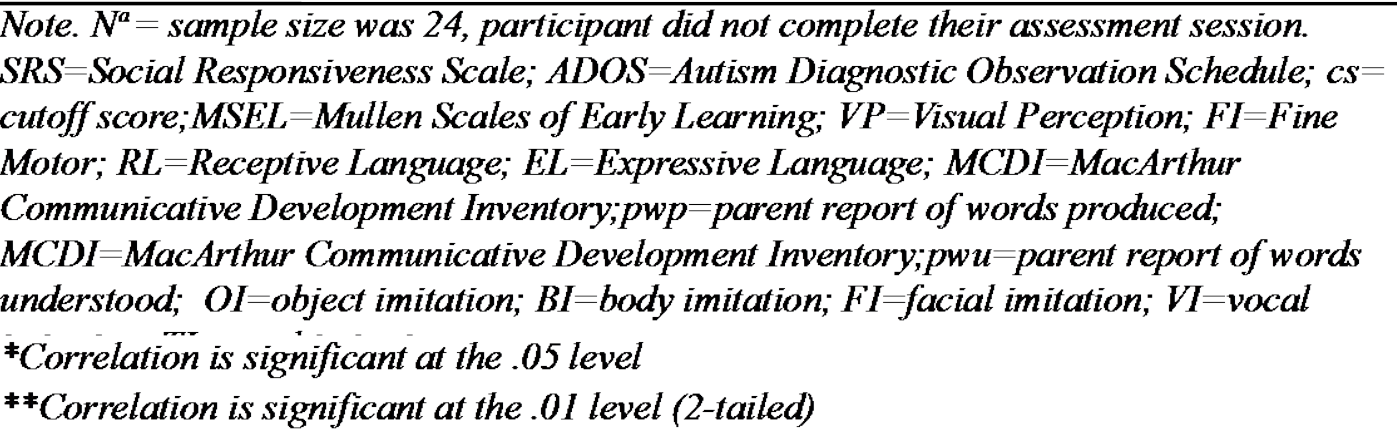 } \\
\hline
\end{tabular}




\section{Hypothesis 7: Imitation performance, as measured by the Motor and Vocal}

\section{Imitation Assessment (MVIA) will significantly predict autism severity, as measured}

\section{by the Autism Diagnostic Observation Schedule (ADOS) and the Social}

\section{Responsiveness Scale (SRS).}

A Pearson product-moment correlation coefficient was computed to assess the relationship between imitation, as measured by the MVIA, and autism severity, as measured by the ADOS. There was a statistically significant negative correlation between imitation and autism severity, $r(22)=-.485, p<.01$, two-tailed. A break down analysis of the correlation between autism severity, as measure by the ADOS and the different types of imitation shows that object imitation is most strongly correlated to autism severity, $r(22)=-.571, p<.01$, two-tailed. Overall, these results indicate that children who are poor imitators tend to exhibit more severe autism symptomology.

Linear regression was also used to predict measures of autism severity from measures of imitation. This analysis demonstrated that imitation significantly predicted autism severity scores, $b=-.244, t(21)=-2.476, p=.022$. Imitation also explained a significant proportion of variance in autism severity scores, $R^{2}=.226, F(1,21)=6.130, p$ $=.022$. The reported $R^{2}=.226$ for the variable one variable two relationship in this model accounted for $22.6 \%$ of the variation. The reported regression coefficient was $b=-.244$ which indicates that the dependent variable (autism severity) is expected to decrease by -.244 unit when the independent variable (imitation) increases by one. In general, we can predict that good imitators have low autism severity, while poor imitators have high autism severity. Figure 5 graphically depicts this relationship relationship between imitation and autism severity. 
A Pearson product-moment correlation coefficient was computed to assess the relationship between imitation, as measured by the MVIA, and autism severity, as measured by the SRS. There was not a statistically significant correlation between imitation and autism severity, $r(23)=-.202, p=.345$, two-tailed.

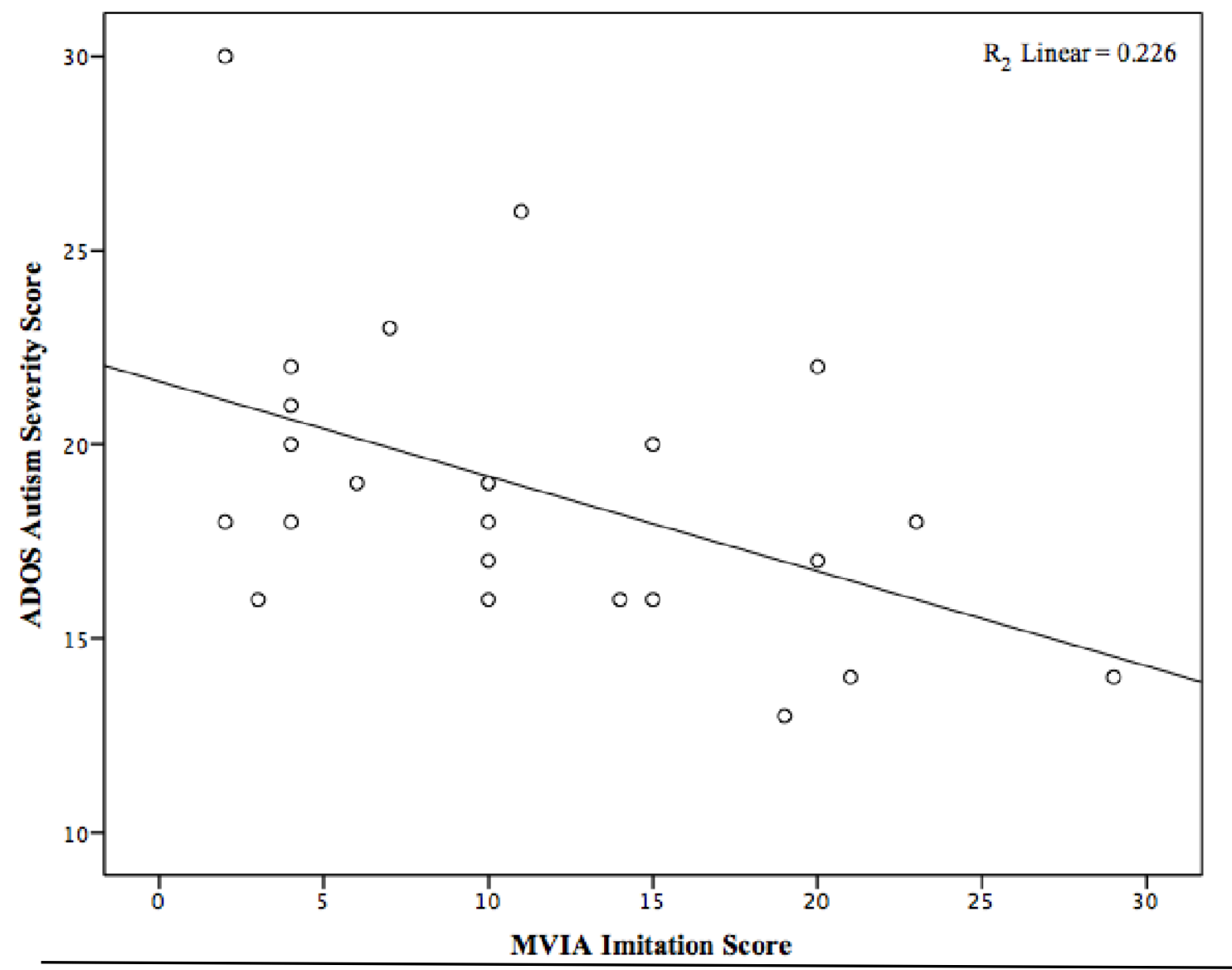

Figure 5. Scatter plot representing the strong negative correlation between imitation and autism severity, as measured by the ADOS.

Hypothesis 8: Imitation performance, as measured by the Motor and Vocal Imitation Assessment (MVIA) will significantly predict language, as measured by 


\section{the receptive and expressive scores in the Mullen Scales of Early Learning (MSEL) and the MacArthur Communicative Development Inventory (MCDI).}

Correlations were conducted in order to assess the relationship between imitation, as measured by the MVIA, and autism severity, as measured by the MSEL scores of expressive and receptive language. There was statistically significant positive correlation between imitation and MSEL scores of expressive language, $r(23)=.585, p<.01$, twotailed. However, there was not a statistically significant correlation between imitation and MSEL scores of receptive language, $r(23)=.275, p=.193$, two-tailed. Further analysis, showed that vocal, $, r(23)=.598, p<.01$, two-tailed and facial imitation, $r(23)$ $=.489, p<.05$, two -tailed were statistically significantly positively correlated to MSEL scores of expressive language. Facial imitation was also significantly positively correlated to MSEL scores of receptive language, $r(23)=.586,, p<.01$, two-tailed. MSEL scores of expressive nor receptive language were correlated to object or body imitation.

Linear regression was again employed to predict measures of MSEL score of expressive language from measures of imitation. This analysis demonstrated that imitation significantly predicted expressive language, $b=.349 t(22)=3.383 p=.003$. Imitation also explained a significant proportion of variance in expressive language, $R^{2}=$ $.342, F(1,22)=11.445, p=.003$. The reported $R^{2}=.342$ for the variable one variable two relationship in this model accounted for $34.2 \%$ of the variation. The reported regression coefficient was $b=.349$ which indicates that the dependent variable (expressive language) is expected to decrease by .349 unit when the independent variable (imitation) increases by one. This implies that children who imitate more are likely to 


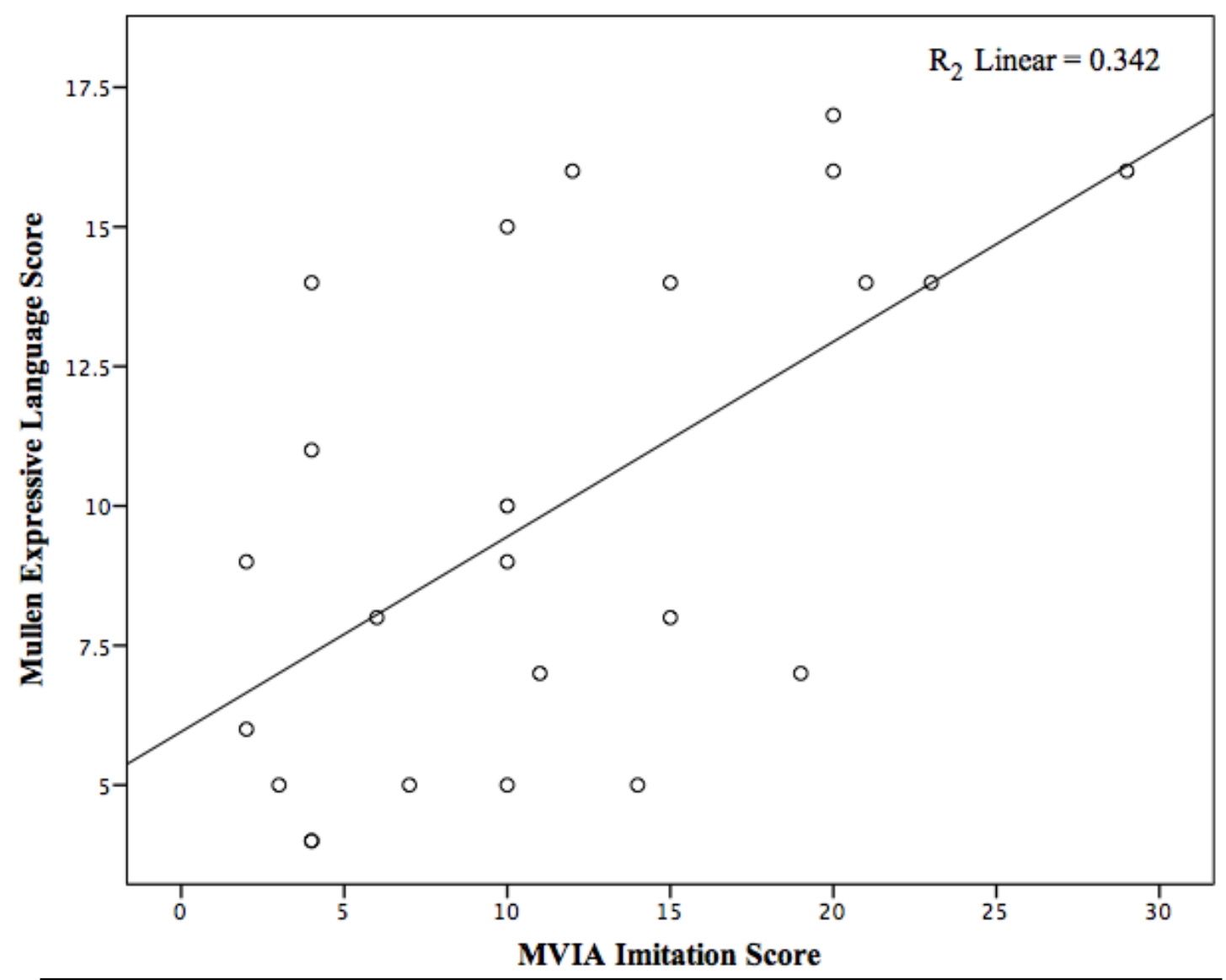

Figure 6. Scatter plot representing the strong positive correlation between imitation and expressive language, as measured by the Mullen.

have more expressive language than children who imitate less. Figure 6 graphically depicts this relationship between imitation and expressive language.

The relationship between imitation and language was further explored by assessing the correlation between imitation, as measured by the MVIA, and expressive and receptive language, as measured by the MCDI. Imitation was correlated to expressive language, as measured by the MCDI, $r(23)=.455, p<.05$, two-tailed but was not correlated to receptive language, $r(23)=.352, p=.10$, two-tailed.

\section{Discussion}

\section{Study 1}


The MVIA was previously developed to assess imitation and guide target selection in imitation intervention. The present study further evaluated the validity of the MVIA as a measure for assessing imitation. As predicted, the results demonstrated that the MVIA pre-treatment measure of imitation had a high internal consistency score and the subscales (object, body, vocal and facial imitation) within the measure were strongly correlated. These results concur with the original study (Espanola, 2014) and further validate the notion that the MVIA serves as a strong measure of imitation. A strong measure of imitation, such as the MVIA, may provide the necessary information for establishing a baseline of imitation skills. This baseline or pre-treatment level of imitation can then better inform clinicians and researchers about the child's current repertoire and possible deficits, information that is critical to formulating an intervention that is appropriate for the child's current skill level.

The sequence of imitation skills yielded by the MVIA protocol was also replicated in the current study. Pre-treatment level of imitation showed that children performed best in imitation tasks involving object imitation, followed by body, vocal and then facial imitation. The ability to replicate this sequence of imitation through pretreatment imitation performance further fosters the notion that the MVIA protocol accurately reflects a hierarchy of imitation skills ordered from simple to complex. In sum, these results establish that the MVIA is a useful measure of imitation and substantiate the postulated instructional sequence in the protocol.

Intervention Outcomes. The purpose of the present study was to evaluate the differences in imitation intervention outcomes between children with ASD randomly assigned to the MVIA treatment group and the VB-MAPP comparison group. Targets for 
intervention for the MVIA treatment group followed the sequence of skills outlined in the MVIA protocol, while the targets for intervention in the VB-MAPP comparison group followed the sequence of skills in the VB-MAPP curriculum guide. The MVIA protocol provides an empirically validated instructional sequence that is organized from simple to complex for teaching imitation to children with ASD. The VB-MAPP is a published curriculum guide that provides a theoretically useful sequence for teaching imitation.

The present study used a discrete trial training procedure with an adult therapist to teach children with ASD to imitate. Research has demonstrated that this strategy is effective for developing imitation skills in children with ASD (e.g., Brown et al., 2008). Thus, it was hypothesized that all participants would demonstrate increased imitation skills after the onset of treatment, as determined by skills that reached the mastery criteria. Visual inspection of the baseline and treatment data confirmed this hypothesis and as expected most children in both groups performed better in imitation tasks during treatment than during baseline. These results are consistent with previous studies (e.g., Smith, 2001) that demonstrate that discrete trial training is successful in developing imitation skills in children with ASD. There were some children whose performance did not improve after the implementation of the treatment. However, the majority of these children were in the VB-MAPP comparison group. These results imply that the lack of improvement were due to the protocol rather than ineffective intervention procedures.

A recent review of interventions targeting imitation suggested that the success of an intervention might not only lie in the type of intervention but in the way in which targets are selected for intervention (Ledford \& Wolery, 2011). Thus, it was hypothesized that participants whose target selection was based on an empirically validated protocol 
(MVIA treatment group) would have a higher number of skills mastered than the participants whose target selection was based on a theoretically grounded curriculum guide (VB-MAPP comparison group). This hypothesis was confirmed. Results showed that participants in the MVIA treatment group had significantly more skill acquisition than participants in the VB-MAPP comparison group.

Skill acquisition was further evaluated by analyzing skill mastery for each type of imitation. Research has never directly analyzed skill acquisition across different types of imitation. Thus, it was difficult to make any formal hypothesis regarding patterns of skill acquisition during intervention. However, it was logical to predict that there would be parallel between the patterns of performance during assessments and patterns of skill acquisition during intervention. Simply stated, if children did well on a specific type of task during their assessment, it was expected that they would demonstrate higher skill acquisition on that type of task during their intervention. As was the case with the present study, pre-treatment imitation performance showed that children performed better in object and body imitation tasks than vocal and facial imitation tasks. This pattern of performance was consistent with the pattern of performance related to skill acquisition. Object imitation tasks had the highest number of skills mastered, followed by body, vocal and then facial imitation. Skill acquisition for the VB-MAPP comparison group also followed a similar sequence as that found in the MVIA protocol. Object and body imitation had the highest number of skills mastered followed by vocal and facial imitation.

This pattern of performance may largely explain why participants in the MVIA treatment group had superior skill acquisition than participants in the VB-MAPP 
comparison group. This pattern of performance suggests that the MVIA protocol most accurately represents a hierarchy of imitation skills ordered from simple to complex. Children the children in the MVIA treatment group started with simple imitation tasks (e.g., banging a hammer) and mastered these skills before gradually moving on to more complex skills. While children in the VB-MAPP comparison group started with the most complex imitation tasks (e.g., opening mouth/saying /ah/) and were often unable to master these skills. These children who never reached the criteria for mastery for the initial imitation tasks were unable to move on to other types of imitation tasks. Therefore, they were never given the opportunity to learn some of the more simple imitation tasks. In essence, setting unachievable goals not only inhibited skill acquisition but also prevented access to other learning opportunities. These findings affirm the importance of choosing goals for intervention that are compatible with the learner's current level of skill and stress the importance of teaching skills using a progressive hierarchy of difficulty.

Skill acquisition for vocal and facial imitation is sometimes emphasized during imitation intervention because these skills are often perceived as indispensable in the development of expressive communication. Accordingly, the present study also evaluated intervention outcomes in terms of performance in vocal and facial imitation. All participants in the VB-MAPP comparison group started with vocal and facial imitation tasks, while all participants in the MVIA treatment group started with object imitation and had to proceed through body imitation in order to reach vocal and then facial imitation. This meant that participants in the VB-MAPP comparison group had the entire duration of the intervention to acquire vocal and facial imitation skills, whereas the participants in the MVIA treatment group had only a portion of that time to acquire vocal 
and facial imitation skills. Despite these differences in the allocation of time, participants in the MVIA treatment group and the VB-MAPP treatment group had similar task mastery on these complex vocal and facial imitation tasks. This indicates that interventions that focus solely on vocal and facial imitation are not more effective or efficient at bringing about these complex skills than interventions that focus on developing a comprehensive imitative repertoire. Furthermore, these results also demonstrate that starting with simple tasks and gradually increasing the difficulty of these tasks more effectively develops a comprehensive imitative repertoire.

Assessing multiple outcome measures is the best method of capturing the success of an intervention. Therefore, in addition to evaluating the effectiveness of the intervention via skill acquisition, efficiency was also assessed by analyzing the ratio of trials to mastery and total trials. Each trial represents a learning opportunity, this ratio provides insight as to how many of the learning opportunities presented actually resulted in skill acquisition. Evaluating learning opportunities is crucial in understanding the efficiency of an intervention because learning opportunities represent resources, such as time, that are allocated towards the intervention.

As hypothesized, the participants in the MVIA treatment group demonstrated more efficient learning. The results showed that more learning opportunities resulted in skill acquisition for the MVIA treatment group, whereas, fewer learning opportunities resulted in skill acquisition for the VB-MAPP comparison group. The number of trials spent on trials that never lead to mastery was also analyzed for the purpose of further evaluating the efficiency of skills acquisition. As hypothesized children in the MVIA treatment group had less trials that never reached the criteria for mastery. Relative to the 
MVIA treatment group, the VB-MAPP comparison group spent twice the amount of time on skills that never reached the criteria for mastery. Moreover, inspection of the individual data demonstrates that some children in the VB-MAPP comparison group spent the entire duration of the intervention on the same tasks without ever reaching criteria for mastery for those tasks.

This information puts into the perspective the overall productivity of the intervention. It shows not only where resources were efficiently used, but also where resources were potentially misused. When learning opportunities do not yield skill acquisition, as occurred often with the VB-MAPP comparison group, then the resources that were allocated to these learning opportunities are wasted. These findings show the value in selecting appropriate targets for intervention and reveal the potential detrimental effects of selecting inappropriate targets for intervention. In general, these results indicate that following a validated instructional sequence, such as that found in the MVIA protocol, may prevent clinicians from selecting targets that are not yet appropriate for the child’s current skill level and thus never result in skill mastery.

The present study also analyzed differences in active responding between the MVIA and the VB-MAPP group. Active responding is defined as trials in which the child either successfully imitated or attempted to imitate the modeled response. Evaluating attempts to imitate may provide insight into emerging imitative responses and the child's motivation to imitate. It was hypothesize that participants in the MVIA group when compared to the participants in the VB-MAPP comparison group would have higher levels of active responding. This hypothesis was confirmed. Children in the MVIA treatment group not only had more successful imitation but also attempted to imitate 
more. These results in part suggest that children in the MVIA treatment group had more motivation and more emerging imitative responses than children in the VB-MAPP comparison group.

Generalization is another important factor in determining the success of an intervention. Generalization refers to the extent to which skills taught in one set of conditions will extend to a novel set of conditions. There are two types of generalization: response generalization and stimulus generalization (Cooper et al., 2007). Response generalization occurs when a functionally similar response emerges without prior training. For instance, if a child is taught to imitate banging a hammer and without prior training then imitates shaking a maraca, response generalization has taken place. Stimulus generalization occurs when the response occurs in the presence of a variety of stimuli within the same class. Following the previous example, stimulus generalization can be said to have occurred if the child imitates banging a variety of hammers, across a variety of settings and from a variety of people. From a clinical standpoint both types of generalization are essential in the success of an intervention, as it allows the child to maximize their learning.

The participants in the MVIA treatment group demonstrated superior generalization of imitation skills over the participants in the VB-MAPP comparison group. Imitation skills for the generalization probes varied across the different types of imitation (e.g., object and body) and the different features of imitation within each type (e.g., meaningful vs. non-meaningful). These results suggest that generalization may have occurred across a variety of categories and features of imitation. However, further analysis may be necessary to uncover whether generalization occurred across specific 
types of imitation. This information may provided insight as to how skill acquisition in a specific category can impact skill acquisition in another category.

To summarize, the results evaluating intervention outcomes revealed that participants in both groups acquired imitation skills. However, participants in the MVIA treatment group acquired substantially more imitation skills and acquired these skills faster and more efficiently than the participants in the VB-MAPP comparison group. Also, participants in the MVIA group spent less time on skills that never reached the criteria for mastery and engaged in more active responding than the participants in the VB-MAPP comparison group. These findings reveal the immense impact that target selection has on the effectiveness and efficiency of an intervention and further illustrate the usefulness of implementing an empirically validated protocol, such as the MVIA, in setting achievable and appropriate goals for intervention.

\section{Study 2}

Amongst the many skills often targeted during early intervention, imitation is particularly important because of the integral role it plays in the development of communication and social skills. The purpose of study two was to analyze the relationship between pre-treatment imitation, as measured by the Motor and Vocal Imitation Assessment (MVIA), and core developmental areas affected by autism.

Imitation as a Predictor. The present study hypothesized that pre-treatment imitation would significantly predict autism severity, as measured by the Autism Diagnostic Observation Schedule (ADOS) and the Social Responsiveness Scale (SRS). Results showed that pre-treatment imitation significantly predicted autism severity, as measured by the ADOS but not the SRS. This disparity may be partly due to the context 
in which the measures are conducted. The ADOS like the MVIA is based on direct observation, thus both more likely to capture behaviors in a clinical setting. While the SRS is based on caregiver report and thus, is more likely to capture the child's behaviors in the natural environment. The similarity between contexts between the ADOS and MVIA may result in the stronger predictive relationship.

Previous studies have also found that despite the SRS and ADOS being reliable and valid measures, there appear to be differences in the ways in which these measures classify and categorize severity (Reszka, Boyd, McBee, Hume, \& Odom, 2014). These results suggest that imitation performance may be one of the many factors that account for some of the incongruity between the measures, indicating that future research should further evaluate this relationship in order to better understand these differences in classification and categorization of autism severity.

These findings were consistent with those found by Rogers and colleagues (2003) but not consistent with findings in Espanola (2014). The most notable distinction between these studies was the participant's level of imitative ability. Participants with ASD in the Rogers et al. (2003) had low levels of imitation, similar to that in the present study, while the participants with ASD in Espanola (2014) did not. These findings suggest that the relationship between imitation and autism severity may change across varying levels of imitative ability. Future research should seek to identify how varying levels of imitation performance may change the relationship between imitation and autism severity. It may be possible that imitation may predict autism severity only when there are deficits in imitation. This possible moderating effect of imitation has yet to be explored and may 
have profound implications for the development of treatments and may also broaden our understanding of how these treatments work and who may benefit from these treatments.

The literature consistently has consistently demonstrated that imitation is deeply intertwined with various aspects of language development (Luyster et al., 2008; Stone et al., 1997; Stone, \& Yoder, 2001; Toth et al., 2006). Therefore, it was hypothesized that imitation performance would predict language, as measured by the receptive and expressive scores in the Mullen: Scales of Early Learning (MSEL) and the MacArthur Communicative Development Inventory (MCDI). Imitation predicted expressive but not receptive language in the MSEL and MCDI. The relationship between imitation and communication skills has considerable support, there have been various studies that have found that imitation predicts both concurrent (McEwen et al., 2007, Sigman \& Ungerer, 1984) and future communication skills (Poon et al., 2011, Stone and Yoder, 2001, Young et al., 2011).

Some studies have specifically evaluated the relationship between different types of imitation and communication. Sigman and Ungerer (1984) found differential patterns of correlations between language and imitation. The results showed that vocal and body imitation were significantly correlated to receptive language but only vocal not body imitation was correlated to expressive language. Stone and colleagues (2003) did not assess correlations between imitation and receptive language, but did find that body imitation was correlated to expressive language. Furthermore, object imitation and expressive language were also evaluated by Stone el al. (2003), but were not significantly correlated. Espanola (2014) found that object, body, vocal and facial imitation were all correlated to expressive and receptive language. In the present study, facial imitation was 
the only type of imitation was significantly correlated to receptive and expressive language. Vocal imitation was only correlated to expressive language. The present findings are comparable to previous studies in some ways but not others. Overall, all these findings indicate that there are some inconsistencies in literature about the relationship between different types of imitation and communication.

These inconsistencies in the literature may be in part due to the inherent nature of hypothesis testing. Hypothesis testing entails a set of statistical procedures used to accept or reject the null hypothesis. The null hypothesis is accepted when there is no effect, and it is rejected when there is an effect. Hypothesis testing always carries the probability of error, type I error denotes the probability of finding an effect when there is not one, while type II error denotes the probability of not finding an effect when there is one. When studies employ statistical analysis, the assumption should be that random samples may sometimes be selected that fail to reject the null hypothesis when the null hypothesis is false (type II error). Simply stated, inconsistencies across studies or failure to replicate when the effect is true should be expected to occur with some probability (Francis, 2012).

The inconsistencies amongst the studies may also be due to the lack of consistency in measures used to assess imitation across the different studies. Most of these studies used different measures to assess imitation, each measure usually had it's own set of procedures and set of behaviors for assessing imitation. This suggests that the inconsistencies across some of the previously discussed findings may be explained by differences in procedures or behaviors used to assesse imitation or perhaps a lack of validity in the measures used to assess imitation. Researchers should attempt to use validated and well-established measures to assess imitation uniformly, as this may help 
reduce some of the factors that may be obscuring or erroneously identifying predictive relationships.

\section{Conclusions}

Imitation is one of the core socio-emotional skills that are often targeted for intervention in children with ASD (National Research Council, 2001). The literature suggests that interventions that are currently being employed may not be as effective due to a lack of protocol for choosing targets for intervention (Ledford \& Wolery, 2011). The present study sought to explore differences in imitation intervention outcomes for children with ASD randomly assigned to two different protocols for target selection. The MVIA treatment group used an empirically validated protocol for target selection, while the VB-MAPP comparison group used a published curriculum guide for target selection. Results suggest that the MVIA treatment group had significantly better intervention outcomes than the VB-MAPP comparison group. The MVIA treatment group showed more skill acquisition and this skill acquisition was more efficient and resulted in more active responding than that of the VB-MAPP comparison group.

The current findings suggest that the MVIA protocol was more effective than the VB-MAPP curriculum guide at increasing imitation skills. Furthermore, these findings imply that the main factor responsible for the effectiveness and efficiency of the MVIA treatment group was the protocol's method for organizing and sequencing skills from simple to complex. These findings strengthen the notion that the sequence of skills in the MVIA protocol lead improved identification of appropriate targets, which in turn increases the effectiveness and efficiency of the intervention. 
The overall success of the MVIA treatment group while striking should still be interpreted cautiously. The value of the protocol not only depends on greater gains in imitation in general but it is also defined by greater gains in complex imitation skills. As previously discussed, interventions that target verbal communication skills often assume that the child can imitate vocal responses. Consequently, when a child does not have a vocal imitation repertoire, interventions that target verbal communication skills are often rendered ineffective. The importance of vocal imitation in verbal communication skills training often leads to an emphasis on vocal imitation during imitation intervention.

The present findings show that skill acquisition for vocal imitation was generally low for both groups. However, as previously discussed this skill was targeted at different time points for each group, thus the MVIA treatment group had less opportunities to acquire vocal imitation than the VB-MAPP group. This temporal incongruence may be masking differences in skill acquisition for vocal imitation between the groups. Future research should consider evaluating the protocols over a longer span of time, so that the participants are given more opportunities to transition from the beginning of the protocol through the end of the protocol.

Generalization and maintenance are two major aspects of an intervention that gauge the success of outcomes for children with ASD. Future research should endeavor to formally evaluate generalization for specific tasks, this analysis may provide critical information about skill acquisition. To date, research indicates that generalization may be restricted within the confines of the type of the imitation trained. This means imitation will generalize within a response class, but not across response classes. For instance, mastery across object imitation tasks would not transition to mastery across body 
imitation tasks (Poulson, Kyparissos, Andreatos, Kymissis, \& Parnes, 2002).

Additionally, research has also demonstrated that generalization from non-verbal to verbal imitation is very difficult (Buddenhagen, 1971). For instance, Garcia, Baer, and Firestone (1971), employed a multiple-baseline design to assess generalization across motor and vocal imitation (small motor, large motor, short vocal, and long vocal). Their results showed that there was a lack of generalization across these different types of imitation. However, these studies did not use a sequence of skills to teach imitation. The use of the sequence of skills in the MVIA protocol may facilitate generalization across categories. Conceivably, training one set of skills (e.g., object imitation) may help lay the foundation for the subsequent skill (e.g., body imitation).

Furthermore, future research should evaluate maintenance. Maintenance provides researchers with critical insight about the retention of skills acquired. The present study did not evaluate maintenance. Consequently, it is difficult to know whether or not skills were maintained after intervention. It may be helpful for future studies to assess maintenance via a formal follow up assessment. Evaluating both generalization and maintenance of skills would provide researchers with a more holistic comprehension of intervention outcomes for each protocol and could also inform clinicians about the possible need for complementing intervention with supplementary procedures for generalization and maintenance.

The purpose of the MVIA protocol and the VB-MAPP curriculum is to serve as a guide for intervention, both of these guides provide critical information about when to begin intervention and which skills to target. The purpose of this study to was to evaluate differences in intervention outcomes due to target selection. A possible limitation of this 
study is the lack of protocol fidelity data. Protocol fidelity may have provided useful information about the extent to which target selection adhered to the procedures for target selection for the respective guides. Thus, future studies seeking to replicate the present findings should endeavor to analyze protocol fidelity. This information would prove to be essential as it directly speaks to the validity and reliability of the factor hypothesized to be responsible for the differences in intervention outcomes.

It is critical to understand that the MVIA protocol should not be implemented in a lock-step progression but rather should be adapted to the unique needs and skills of each child. This may be especially important for children with ASD because research has established that children with ASD commonly have an uneven or scattered profile of skill development (CDC, 2014). As such, an appropriate and individualized intervention plan can only be achieved when the unique skill deficits of the child are assessed and taken into consideration during target selection. Therefore, it is important to use the MVIA and the MVIA protocol in conjunction, so to not only understand how to proceed with the intervention but where to begin the intervention. Accordingly, clinicians are encouraged to implement the MVIA protocol with flexibility and practicality. The success of the MVIA and MVIA protocol will ultimately be measured by the applicability and acceptability of the protocol's methods and procedures during practical application. 


\section{REFERENCES}

Abravanel, E., Levan-Goldschmidt, E., \& Stevenson, M.B. (1976). Action imitation: The early phase of infancy. Child Development, 47(4), 1032-1044.

Allen, G., \& Courchesne, E. (2014). Differential effects of developmental cerebellar abnormality on cognitive and motor functions in the cerebellum: an fMRI study of autism. The American Journal of Psychiatry, 160(2), 262-273.

Amendah, D., Grosse, S. D., Peacock, G., \& Mandell, D. S. (2011). The economic costs of autism: A review. Autism Spectrum Disorders, 1347-1360.

American Psychiatric Association. (2013). Diagnostic and statistical manual of mental disorders (5 ${ }^{\text {th }}$ ed.). Arlington, VA: American Psychiatric Publishing.

Baer, D. M., Peterson, R. F., \& Sherman, J. A. (1967). The development of imitation by reinforcing behavioral similarity to a model. Journal of the Experimental Analysis of Behavior, 10(5), 405-416.

Baer, D. M., Wolf, M. M., \& Risley, T. R. (1968). Some Current Dimensions Of Applied Behavior Analysis. Journal of Applied Behavior Analysis, 1(1), 91-97.

Bandura, A. (1977). Social learning theory. Englewood Cliffs, NJ: Prentice Hall.

Bellg, A. J., Borrelli, B., Resnick, B., Hecht, J., Minicucci, D. S., Ory, M., ... \& Czajkowski, S. (2004). Enhancing treatment fidelity in health behavior change studies: Best practices and recommendations from the NIH Behavior Change Consortium. Health Psychology, 23(5), 443.

Blake, R., Turner, L. M., Smoski, M. J., Pozdol, S. L., \& Stone, W. L. (2003). Visual recognition of biological motion is impaired in children with autism.

Psychological Science, 14(2), 151-157.

Borrelli, B., Sepinwall, D., Ernst, D., Bellg, A. J., Czajkowski, S., Breger, R., ... \& Orwig, D. (2005). A new tool to assess treatment fidelity and evaluation of treatment fidelity across 10 years of health behavior research. Journal of Consulting and Clinical Psychology, 73(5), 852.

Bourret, J., Vollmer, T. R., \& Rapp, J. T. (2004). Evaluation of a vocal mand assessment and vocal mand training procedures. Journal of Applied Behavior Analysis, 37(2), 129-144.

Boyle, C. L., \& Lutzker, J. R. (2005). Teaching young children to discriminate abusive from nonabusive situations using multiple exemplars in a modified discrete trial teaching format. Journal of Family Violence, 20(2), 55-69. 
Brown, A. K., Brown, J. L., \& Poulson, C. L. (2008). Discriminating which fork to use: Teaching selective imitation to people with autism. Research in Autism Spectrum Disorders, 2(2), 199-208.

Bryson, S. E., Zwaigenbaum, L., Brian, J., Roberts, W., Szatmari, P., Rombough, V., \& McDermott, C. (2007). A prospective case series of high-risk infants who developed autism. Journal of Autism and Developmental Disorders, 37(1), 12-24.

Cardon, T. A., \& Wilcox, M. J. (2011). Promoting imitation in young children with autism: A comparison of reciprocal imitation training and video modeling. Journal of Autism and Developmental Disorders, 41(5), 654-666.

Carr, E. G., \& Darcy, M. (1990). Setting generality of peer modeling in children with autism. Journal of Autism and Developmental Disorders, 20(1), 45-59. doi: 10.1007/BF02206856

Carr, J. E., Nicolson, A. C., \& Higbee, T. S. (2000). Evaluation of a brief multiplestimulus preference assessment in a naturalistic context. Journal of Applied Behavior Analysis, 33(3), 353-357.

Catania C. A. (1972). Learning (3rd ed.) Engelwood Cliffs, NJ: Prentice-Hall.

Centers for Disease Control and Prevention, Autism Spectrum Disorders (ASDs) Data and Statistics (2014). Retrieved from http://www.cdc.gov/ncbddd/autism/data.html.

Charman, T., Baron-Cohen, S., Swettenham, J., Baird, G., Cox, A., \& Drew, A. (2000). Testing joint attention, imitation, and play as infancy precursors to language and theory of mind. Cognitive development, 15(4), 481-498.

Charman, T., Baron-Cohen, S., Swettenham, J., Baird, G., Drew, A., \& Cox, A. (2003). Predicting language outcome in infants with autism and pervasive developmental disorder. International Journal of Language \& Communication Disorders, 38(3), 265-285.

Charman, T., Swettenham, J., Baron-Cohen, S., Cox, A., Baird, G., \& Drew, A. (1997). Infants with autism: An investigation of empathy, pretend play, joint attention, and imitation. Developmental Psychology, 33(5), 781-789.

Chaste, P., \& Leboyer, M. (2012). Autism risk factors: genes, environment, and geneenvironment interactions. Dialogues in Clinical Neuroscience, 14(3), 281-292.

Constantino, J. N. (2002). Social Responsiveness Scale-Adult Version (SRS). Los Angeles, CA: Western Psychological Services. 
Cooper, J. O., Heron, T. E., \& Heward, W. L. (2007). Applied Behavior Analysis (2nd ed.). Upper Saddle River, NJ: Prentice Hall.

Davis, T. N., Dacus, S., Strickland, E., Machalicek, W., \& Coviello, L. (2013). Reduction of automatically maintaine self-injurious behavior utilizing noncontingent matched stimuli. Developmental Neurorehabilitation, 16(3), 166-171.

Dawson, G., \& Adams, A. (1984). Imitation and social responsiveness in autistic children. Journal of Abnormal Child Psychology, 12(2), 209-225.

Dawson, G., Carver, L., Meltzoff, A. N., Panagiotides, H., McPartland, J., \& Webb, S. J. (2002). Neural correlates of face and object recognition in young children with autism spectrum disorder, developmental delay, and typical development. Child Development, 73(3), 700-717.

Dawson, G., Jones, E. J., Merkle, K., Venema, K., Lowy, R., Faja, S., \& Webb, S. J. (2012). Early behavioral intervention is associated with normalized brain activity in young children with autism. Journal of the American Academy of Child \& Adolescent Psychiatry, 51(11), 1150-1159.

Dawson, G., Meltzoff, A. N., Osterling, J., Rinaldi, J., \& Brown, E. (1998). Children with autism fail to orient to naturally occurring social stimuli. Journal of Autism and Developmental Disorders, 28(6), 479-485.

Dawson, G., Rogers, S., Munson, J., Smith, M., Winter, J., Greenson, J., \& Varley, J. (2010). Randomized, controlled trial of an intervention for toddlers with autism: the Early Start Denver Model. Pediatrics, 125(1), e17-e23.

Dawson, G., Webb, S. J., \& McPartland, J. (2005). Understanding the nature of face processing impairment in autism: insights from behavioral and electrophysiological studies. Developmental Neuropsychology, 27(3), 403-424.

De Giacomo, A., Portoghese, C., Martinelli, D., Fanizza, I., L’Abate, L., \& Margari, L. (2009). Imitation and communication skills development in children with pervasive developmental disorders. Neuropsychiatric Disease and Treatment, 5, 355-362.

Dietert, R. R., Dietert, J. M., \& DeWitt, J. C. (2011). Environmental risk factors for autism. Emerging Health Threats Journal, 4.

Digennaro, F. D., Martens, B. K., \& Kleinmann, A. E. (2007). A Comparison Of Performance Feedback Procedures on Teachers Treatment Implementation Integrity And Students' Inappropriate Behavior In Special Education Classrooms. Journal Of Applied Behavior Analysis, 40(3), 447-461. 
Eikeseth, S. (2009). Outcome of comprehensive psycho-educational interventions for young children with autism. Research in Developmental Disabilities, 30(1), 158178.

Eldevik, S., Hastings, R. P., Hughes, J. C., Jahr, E., Eikeseth, S., \& Cross, S. (2009). Meta-analysis of early intensive behavioral intervention for children with autism. Journal of Clinical Child \& Adolescent Psychology, 38(3), 439-450.

Espanola, E. (2014). Assessment Of Motor And Vocal Imitation (Unpublished master's thesis). Florida International University, Miami, Florida.

Fenson, L., Dale, P. S., Reznick, J. S., Thal, D., Bates, E., Hartung, J. P., Pethick, S., \& Reilly, J. S. (1993). The MacArthur Communicative Development Inventories: User's guide and technical manual. San Diego, CA: Singular Publishing Group.

Folstein, S., \& Rutter, M. (1977). Genetic influences and infantile autism. Nature, 265(5596), 726-728.

Francis, G. (2012). The psychology of replication and replication in psychology. Perspectives on Psychological Science, 7(6), 585-594.

Gardener, H., Spiegelman, D., \& Buka, S. L. (2011). Perinatal and neonatal risk factors for autism: A comprehensive meta-analysis. Pediatrics, 128(2), 344-355.

Garfinkle, A. N., \& Schwartz, I. S. (2002). Peer imitation increasing social interactions in children with autism and other developmental disabilities in inclusive preschool classrooms. Topics in Early Childhood Special Education, 22(1), 26-38.

Gewirtz, J. L. (1969). Mechanisms of social learning: Some roles of stimulation and behavior in early human development. Handbook of Socialization Theory and Research, 57-212.

Glendenning, N. J., Adams, G. L., \& Sternberg, L. (1983). Comparison of prompt sequences. American Journal of Mental Deficiency, 88(3), 321-325.

Glenn, S. S., Ellis, J., \& Greenspoon, J. (1992). On the revolutionary nature of the operant as a unit of behavioral selection. American Psychologist, 47(11), 13291336.

Green, G. (2001). Behavior analytic instruction for learners with autism: Advances in stimulus control technology. Focus on Autism and Other Developmental Disabilities, 16(2), 72-85.

Green, G., Brennan, L. C., \& Fein, D. (2002). Intensive behavioral treatment for a toddler at high risk for autism. Behavior Modification, 26(1), 69-102. 
Guillaume, P. (1971). Imitation in children. Chicago: University of Chicago Press.

Guthrie, W., Swineford, L. B., Nottke, C., \& Wetherby, A. M. (2013). Early diagnosis of autism spectrum disorder: stability and change in clinical diagnosis and symptom presentation. Journal of Child Psychology and Psychiatry, 54(5), 582-590.

Hallmayer, J., Cleveland, S., Torres, A., Phillips, J., Cohen, B., Torigoe, T., ... \& Risch, N. (2011). Genetic heritability and shared environmental factors among twin pairs with autism. Archives of General Psychiatry, 68(11), 1095-1102.

Hattier, M. A., \& Matson, J. L. (2012). An examination of the relationship between communication and socialization deficits in children with autism and PDDNOS. Research in Autism Spectrum Disorders, 6(2), 871-880.

Helt, M., Kelley, E., Kinsbourne, M., Pandey, J., Boorstein, H., Herbert, M., \& Fein, D. (2008). Can children with autism recover? If so, how?. Neuropsychology Review, 18(4), 339-366.

Heyes, C. (2010). Mesmerising mirror neurons. Neuroimage, 51(2), 789-791.

Horovitz, M., \& Matson, J. L. (2010). Communication deficits in babies and infants with autism and pervasive developmental disorder-not otherwise specified (PDDNOS). Developmental Neurorehabilitation, 13(6), 390-398.

Howlin, P., Magiati, I., \& Charman, T. (2009). Systematic review of early intensive behavioral interventions for children with autism. Journal Information, 114(1), 23-41.

Ingersoll, B. (2012). Brief report: Effect of a focused imitation intervention on social functioning in children with autism. Journal of Autism and Developmental Disorders, 42(8), 1768-1773.

Ingersoll, B. (2008). The social role of imitation in autism: Implications for the treatment of imitation deficits. Infants \& Young Children, 21(2), 107-119.

Ingersoll, B., \& Schreibman, L. (2006). Teaching reciprocal imitation skills to young children with autism using a naturalistic behavioral approach: Effects on language, pretend play, and joint attention. Journal of Autism and Developmental Disorders, 36(4), 487-505.

Ingersoll, B., Schreibman, L., \& Tran, Q. H. (2003). Effect of sensory feedback on immediate object imitation in children with autism. Journal of Autism and Developmental Disorders, 33(6), 673-683.

Johnston, J. M., \& Pennypacker, H. S. (2009). Strategies and tactics of behavioral research (3rd ed.). New York, NY: Routledge. 
Jones, S. S. (2007). Imitation in infancy the development of mimicry. Psychological Science, 18(7), 593-599.

Kanner, L. (1943). Autistic disturbances of affective contact. Acta paedopsychiatrica, 35(4), 100-136.

Kasari, C., Paparella, T., Freeman, S., \& Jahromi, L. B. (2008). Language outcome in autism: randomized comparison of joint attention and play interventions. Journal of Consulting and Clinical Psychology, 76(1), 125-137.

Kazdin, A. (2013). Single-case research designs ( $2^{\text {nd }}$ ed.). New York: Oxford University Press.

Landrigan, P. J., Lambertini, L., \& Birnbaum, L. S. (2012). A research strategy to discover the environmental causes of autism and neurodevelopmental disabilities. Environmental Health Perspective, 120(7), a258-a260.

Larsson, H. J., Eaton, W. W., Madsen, K. M., Vestergaard, M., Olesen, A. V., Agerbo, E., ... \& Mortensen, P. B. (2005). Risk factors for autism: Perinatal factors, parental psychiatric history, and socioeconomic status. American Journal of Epidemiology, 161(10), 916-925.

Leaf, R., \& McEachin, J. (1999). A work in progress: Behavior management strategies and a curriculum for intensive behavioral treatment of autism. New York, NY: DRL Books.

Ledford, J. R., Gast, D. L., Luscre, D., \& Ayres, K. M. (2008). Observational and incidental learning by children with autism during small group instruction. Journal of Autism and Developmental Disorders, 38(1), 86-103.

Lewis, M. H. (2004). Environmental complexity and central nervous system development and function. Mental Retardation and Developmental Disabilities Research Reviews, 10(2), 91-95.

Logan, K. R., Jacobs, H. A., Gast, D. L., Smith, P. D., Daniel, J., \& Rawls, J. (2001). Preference and reinforcers for students with profound multiple disabilities: Can we identify them? Journal of Developmental and Physical Disabilities, 13(2), 97122.

Lord, C., Rutter, M., \& Le Couteur, A. (1994). Autism Diagnostic Interview-Revised: A revised version of a diagnostic interview for caregivers of individuals with possible pervasive developmental disorders. Journal of Autism and Developmental Disorders, 24(5), 659-685. 
Lovaas, O. I. (1981). Teaching developmentally disabled children: The ME book. Austin, TX: PROED.

Lovaas, O. I. (2003). Teaching individuals with developmental delays: Basic intervention techniques. Austin, TX: PROED.

Lovaas, O. I. (1977). The autistic child: Language development through behavior modification. New York: Irvington.

Luyster, R. J., Kadlec, M. B., Carter, A., \& Tager-Flusberg, H. (2008). Language assessment and development in toddlers with autism spectrum disorders. Journal of Autism and Developmental Disorders, 38(8), 1426-1438.

MacDuff, G. S., Krantz, P. J., \& McClannahan, L. E. (2001). Prompts and prompt-fading strategies for people with autism. Making a Difference: Behavioral Intervention for Autism, 37-50.

Macintosh, K. E., \& Dissanayake, C. (2004). The similarities and differences between autistic disorder and asperger's disorder: A review of the empirical evidence. Journal of Child Psychology and Psychiatry, 45(3), 421-434.

McCoy, K., \& Hermansen, E. (2007). Video modeling for individuals with autism: A review of model types and effects. Education and Treatment of Children, 30(4), 183-213. doi:10.1353/etc.2007.0029

McGhan, A. C., \& Lerman, D. C. (2013). An assessment of error-correction procedures for learners with autism. Journal of Applied Behavior Analysis, 46(3), 626-639.

Matson, J. L., Kozlowski, A. M., Hattier, M. A., Horovitz, M., \& Sipes, M. (2012). DSM-IV vs DSM-5 diagnostic criteria for toddlers with autism. Developmental Neurorehabilitation, 15(3), 185-190.

Masur, E. F., \& Ritz, E. G. (1984). Patterns of gestural, vocal, and verbal imitation performance in infancy. Merrill-Palmer Quarterly 30(4), 369-392.

Mazur, J. E. (2000). Tradeoffs among delay, rate, and amount of reinforcement. Behavioural Processes, 49(1), 1-10.

McEwen, F., Happe, F., Bolton, P., Rijsdijk, F., Ronald, A., \& Dworzynski, K. (2007). Origins of individual difference in imitation: Links with language, pretend play, and socially insightful behavior in two-year-old twins. Child Development, 78(2), 474-492. 
Meindl, J. N., \& Cannella-Malone, H. I. (2011). Initiating and responding to joint attention bids in children with autism: A review of the literature. Research in Developmental Disabilities, 32(5), 1441-1454.

Meltzoff, A. N. (2005). Imitation and other minds: The "like me" hypothesis. Perspectives on Imitation: From Neuroscience to Social Science, 2, 55-77.

Miniscalco, C., Rudling, M., Rastam, M., Gillberg, C., \& Johnels, J. A. (2014). Imitation (rather than core language) predicts pragmatic development in young children with ASD: a preliminary longitudinal study using CDI parental reports. International Journal of Language \& Communication Disorders, 49(3), 369-375.

Mundy, P., Sigman, M., \& Kasari, C. (1990). A longitudinal study of joint attention and language development in autistic children. Journal of Autism and Developmental Disorders, 20(1), 115-128.

Mullen, E.M. (1995). Mullen: Scales of Early Learning (AGS edn). Circle Pines, MN: American Guideline Service, Inc.

Nadel, J., Carchon, I., Kervella, C., Marcelli, D., \& Réserbat- Plantey, D. (1999). Expectancies for social contingency in 2- month- olds. Developmental Science, 2(2), 164-173.

National Institute of Health. (2008). FY 2007 NIH Autism Portfolio. Retrieved from http://www.nimh.nih.gov/health/topics/autism-spectrum-disorders-asd/nihinitiatives/fy-2007-nih-asd-research-portfolio-summary-by-research-area.shtml

Newschaffer, C. J., Croen, L. A., Fallin, M. D., Hertz-Picciotto, I., Nguyen, D. V., Lee, N. L., ... \& Shedd-Wise, K. M. (2012). Infant siblings and the investigation of autism risk factors. Journal of Neurodevelopmental Disorders, 4(1), 7-7.

Nikopoulos, C. K., \& Keenan, M. (2007). Using video modeling to teach complex social sequences to children with autism. Journal of Autism and Developmental Disorders, 37(4), 678-693.

Numis, A. L., Major, P., Montenegro, M. A., Muzykewicz, D. A., Pulsifer, M. B., \& Thiele, E. A. (2011). Identification of risk factors for autism spectrum disorders in tuberous sclerosis complex. Neurology, 76(11), 981-987.

Obhi, S. S., \& Sebanz, N. (2011). Moving together: toward understanding the mechanisms of joint action. Experimental Brain Research, 211(3), 329-336.

Ozonoff, S., Heung, K., Byrd, R., Hansen, R., \& Hertz-Picciotto, I. (2008). The onset of autism: Patterns of symptom emergence in the first years of life. Autism Research, 1(6), 320-328. 
Ozonoff, S., Young, G. S., Belding, A., Hill, M., Hill, A., Hutman, T., ... \& Iosif, A. M. (2014). The broader autism phenotype in infancy: When does it emerge?. Journal of the American Academy of Child \& Adolescent Psychiatry, 53(4), 398-407.

Over, H., \& Carpenter, M. (2013). The social side of imitation. Child Development Perspectives, 7(1), 6-11.

Pace, G. M., Ivancic, M. T., Edwards, G. L., Iwata, B. A., \& Page, T. J. (1985). Assessment of stimulus preference and reinforcer value with profoundly retarded individuals. Journal of Applied Behavior Analysis, 18(3), 249-255.

Partington, J. W. (2008). The Assessment of Basic Language and Learning Skills-Revised (The ABLLS-R). Pleasant Hills, CA: Behavior Analysts.

Piaget, J. (1951). Play, dreams, and imitation in childhood. New York: Routledge.

Pickard, K. E., \& Ingersoll, B. R. (2015). Brief Report: High and low level initiations of joint attention, and response to joint attention: Differential relationships with language and imitation. Journal of Autism and Developmental Disorders, 45(1), 262-268.

Plavnick, J. B., \& Hume, K. A. (2014). Observational learning by individuals with autism: A review of teaching strategies. Autism, 18(4), 458-466.

Poon, K. K., Watson, L. R., Baranek, G. T., \& Poe, M. D. (2012). To what extent do joint attention, imitation, and object play behaviors in infancy predict later communication and intellectual functioning in ASD?. Journal of Autism and Developmental Disorders, 42(6), 1064-1074.

Prevalence of Autism Spectrum Disorders - Autism and Developmental Disabilities Monitoring Network, 14 Sites, United States, 2008. (2012). MMWR Surveillance Summaries, 61(3), 1-19 19p.

Rachman, S. (1972). Clinical applications of observational learning imitation and modeling. Behavior Therapy, 3(3), 379-397.

Ratajczak, H. V. (2011). Theoretical aspects of autism: Causes-A review. Journal of Immunotoxicology, 8(1), 68-79.

Ray, E., \& Heyes, C. (2011). Imitation in infancy: The wealth of the stimulus. Developmental Science, 14(1), 92-105.

Reszka, S. S., Boyd, B. A., McBee, M., Hume, K. A., \& Odom, S. L. (2014). Brief report: Concurrent validity of autism symptom severity measures. Journal of Autism and Developmental Disorders, 44(2), 466-470. 
Rogers, S. J. (2004). Developmental regression in autism spectrum disorders. Mental Retardation and Developmental Disabilities Research Reviews, 10(2), 139-143.

Rogers, S. J., Bennetto, L., McEvoy, R., \& Pennington, B. F. (1996). Imitation and pantomime in high- functioning adolescents with autism spectrum disorders.

Child Development, 67(5), 2060-2073.

Rogers, S. J., Hepburn, S. L., Stackhouse, T., \& Wehner, E. (2003). Imitation performance in toddlers with autism and those with other developmental disorders. Journal of Child Psychology and Psychiatry and Allied Disciplines, 44(5), 763-781.

Rogers, S. J., \& Pennington, B. F. (1991). A theoretical approach to the deficits in children with autism. Development and Psychopathology, 3(02), 137-162.

Rogers S.J., Young, G.S., Cook I., Giolzetti, A., \& Ozonoff S. (2010) Imitating actions on objects in early-onset and regressive autism: Effects and implications of task characteristics on performance. Developmental Psychopathology, 22(1): 71-85.

Rogers, S. J., \& Vismara, L. A. (2008). Evidence-based comprehensive treatments for early autism. Journal of Clinical Child and Adolescent Psychology, 37(1), 8-38.

Ronald, A., \& Hoekstra, R. (2014). Progress in understanding the causes of autism spectrum disorders and autistic traits: Twin studies from 1977 to the present day. In S.H. Rhee \& A. Ronald (Eds.), Behavior genetics of psychopathology (pp. 3365). New York: Springer

Rosales-Ruiz, J., \& Baer, D. M. (1997). Behavioral cusps: A developmental and pragmatic concept for behavior analysis. Journal of Applied Behavior Analysis, 30(3), 533-544.

Rutter, M. (2000). Genetic studies of autism: from the 1970s into the millennium. Journal of Abnormal Child Psychology, 28(1), 3-14.

Sacrey, L. A. R., Bryson, S. E., \& Zwaigenbaum, L. (2013). Prospective examination of visual attention during play in infants at high-risk for autism spectrum disorder: A longitudinal study from 6 to 36 months of age. Behavioural Brain Research, 256(1), 441-450.

Sallows, G. O., and Graupner, T. D. (2005). Intensive behavioral treatment for children with autism: Four-year outcome and predictors. American Journal on Mental Retardation, 110(6), 417-438. 
Schanding Jr, G. T., Nowell, K. P., \& Goin-Kochel, R. P. (2012). Utility of the social communication questionnaire-current and social responsiveness scale as teacherreport screening tools for autism spectrum disorders. Journal of Autism and Developmental Disorders, 42(8), 1705-1716.

Seneff, S., Davidson, R. M., \& Liu, J. (2012). Empirical data confirm autism symptoms related to aluminum and acetaminophen exposure. Entropy, 14(11), 2227-2253.

Sigman, M., \& Ungerer, J. A. (1984). Cognitive and language skills in autistic, mentally retarded, and normal children. Developmental Psychology, 20(2), 293-302.

Simonoff, E. (2012). Autism spectrum disorder: prevalence and cause may be bound together. The British Journal of Psychiatry, 201(2), 88-89.

Skinner, B. F. (1957). Verbal behavior. Upper Saddle River, NJ: Pearson Prentice Hall

Skinner, B. F. (1953). Science and human behavior. New York: MacMillan.

Smith, T. (2001). Discrete trial training in the treatment of autism. Focus on Autism and Other Developmental Disabilities, 16(2), 86-92.

Smith, T. (1999). Outcome of early intervention for children with autism. American Psychological Association, 6(1), 33-49.

Smith, I. M., \& Bryson, S. E. (1994). Imitation and action in autism: a critical review. Psychological bulletin, 116(2), 259=273.

Smith, T., Groen, A. D., \& Wynn, J. W. (2000). Randomized trial of intensive early intervention for children with pervasive developmental disorder. American Journal on Mental Retardation, 105(4), 269-285.

Stone, W. L. Ousley, O. Y. Littleford , C. L. (1997). Motor imitation in young children with autism: What's the object? Journal of Abnormal Child Psychology, 25(6), 475-485.

Stone, W. L., \& Yoder, P. J. (2001). Predicting spoken language level in children with autism spectrum disorders. Autism, 5(4), 341-361.

Sundberg, M. L. (2008) Verbal behavior milestones assessment and placement program: The VB-MAPP. Concord, CA: AVB Press.

Thalheimer, W., \& Cook, S. (2002, August). How to calculate effect sizes from published research articles: A simplified methodology. Retrieved August 27, 2015 from http://work-learning.com/effect_sizes.htm. 
Tomasello, M. (1995). Joint attention as social cognition. Joint Attention: Its Origins and Role in Development, 103-130.

Toth, K., Munson, J., Meltzoff, A. N., \& Dawson, G. (2006). Early predictors of communication development in young children with autism spectrum disorder: Joint attention, imitation, and toy play. Journal of Autism and Developmental Disorders, 36(8), 993-1005.

Trevarthen, C., Kokkinaki, T., \& Fiamenghi, G. A, Jr. (1999). What infants' imitations communicate: With mothers, with fathers and with peers. In J. Nadel \& G. Butterworth (Eds.), Imitation in Infancy (pp. 127-185). Cambridge: Cambridge University Press.

Uzgiris, I.C., \& Hunt, J. McV. (1975). Assessment in infancy: Ordinal scales of psychological development. Champaign, IL: University of Illinois Press.

Vanvuchelen, M., Roeyers, H., \& De Weerdt, W. (2007). Nature of motor imitation problems in school-aged boys with autism A motor or a cognitive problem? Autism, 11(3), 225-240.

Ventola, P., Saulnier, C. A., Steinberg, E., Chawarska, K., \& Klin, A. (2014). Earlyemerging social adaptive skills in toddlers with autism spectrum disorders: An item analysis. Journal of Autism and Developmental Disorders, 44(2), 283-293.

Vismara, L. A., \& Rogers, S. J. (2010). Behavioral treatments in autism spectrum disorder: What do we know?. Annual Review of Clinical Psychology, 6, 447-468.

Vivanti, G., Trembath, D., \& Dissanayake, C. (2014). Mechanisms of imitation impairment in autism spectrum disorder. Journal of Abnormal Child Psychology, 42(8), 1395-1405.

Vollmer, T. R., Sloman, K. N., \& Pipkin, C. S. P. (2008). Practical implications of data reliability and treatment integrity monitoring. Behavior Analysis in Practice, 1(2), 4-11.

Wan, M. W., Green, J., Elsabbagh, M., Johnson, M., Charman, T., \& Plummer, F. (2013). Quality of interaction between at-risk infants and caregiver at $12-15$ months is associated with 3-year autism outcome. Journal of Child Psychology and Psychiatry, 54(7), 763-771.

Warren, Z., McPheeters, M. L., Sathe, N., Foss-Feig, J. H., Glasser, A., \& VeenstraVanderWeele, J. (2011). A systematic review of early intensive intervention for autism spectrum disorders. Pediatrics, 127(5), e1303-e1311. 
Warreyn, P., Paelt, S., \& Roeyers, H. (2014). Social- communicative abilities as treatment goals for preschool children with autism spectrum disorder: The importance of imitation, joint attention, and play. Developmental Medicine \& Child Neurology, 56(8), 712-716.

Watkins, M. W., \& Pacheco, M. (2000). Interobserver agreement in behavioral research: Importance and calculation. Journal of Behavioral Education, 10(4), 205-212.

Williams, J. H., Whiten, A., \& Singh, T. (2004). A systematic review of action imitation in autistic spectrum disorder. Journal of Autism and Developmental Disorders, 34(3), 285-299.

Wong, C., Odom, S. L., Hume, K. A., Cox, A. W., Fettig, A., Kucharczyk, S., ... \& Schultz, T. R. (2015). Evidence-based practices for children, youth, and young adults with autism spectrum disorder: A comprehensive review. Journal of autism and developmental disorders, 45(7), 1951-1966.

Young, J. M., Krantz, P. J., McClannahan, L. E., \& Poulson, C. L. (1994). Generalized Imitation and Response- Class Formation In Children With Autism. Journal of Applied Behavior Analysis, 27(4), 685-697.

Young, G. S., Rogers, S. J., Hutman, T., Rozga, A., Sigman, M., \& Ozonoff, S. (2011). Imitation from 12 to 24 months in autism and typical development: A longitudinal Rasch analysis. Developmental Psychology, 47(6), 1565-1578. 


\begin{tabular}{|l|l|}
\hline FIU IRB Approval: & $10 / 2 / 2013$ \\
\hline FIU IRB Expiration: & $10 / 2 / 2014$ \\
\hline FIU IRB Number: & IRB-13-0416 \\
\hline
\end{tabular}

\title{
$1=10 \mid \begin{aligned} & \text { FLORIDA } \\ & \text { INTERNATIONAL }\end{aligned}$ UNIVERSITY
}

\section{PARENTAL CONSENT TO PARTICIPATE IN A RESEARCH STUDY}

\author{
Intervention of Motor and Vocal Imitation
}

\section{PURPOSE OF THE STUDY}

You and your child are being asked to participate in a research study. This study seeks to provide an intervention package to target motor and vocal imitation in young children with autism spectrum disorders (ASD).

\section{NUMBER OF STUDY PARTICIPANTS}

If you agree to allow your child to participate in this study, he/she and you will be one of 60 people in this research study.

\section{DURATION OF THE STUDY}

Your participation will require that you complete questionnaires related to your child's current skills and abilities. Your child's participation will require that he/she attend sessions conducted 4-5 times per week for up to an hour each session. Participation in the study will not exceed a total of 8 months.

\section{PROCEDURES}

If your child participates in this study, we will ask you and your child to do the following things:

1. Pre-assessment: Your child will be tested and observed to get information on his/her social, language, play, imitation, and nonverbal cognitive skills (or the ability to solve problems that don't involve any language). Additionally, you will be asked to complete assessments about the participants to get information on the previously stated skills, and intervention history.

2. All sessions will be video recorded for later coding.

3. Participants will all receive intervention that is based on applied behavioral analytic principles and delivered by trained therapists.

4. Participants will be randomly assigned to 1 of 2 groups: (1) Intervention as usual, in which appropriate target imitative behaviors will be chosen without a specific order; or (2) intervention with protocol, in which appropriate target imitative behaviors are chosen following a protocol for choosing imitation tasks.

\section{RISKS AND/OR DISCOMFORTS}

The following risks may be associated with your child's participation in this study:

We anticipate no more than a minimal risk in participating in this study. These minimal risks would not exceed those would be encountered in educational settings. Therefore, the benefits of the knowledge learned outweigh any risks. During the course of the study physical guidance may be used, the therapist will use the least amount of guidance necessary to help the learner engage in the target response. Sessions will be discontinued if any problem behaviors present a risk of injury to the child or anyone else in the study or if the parent feels the child is not comfortable. 


\begin{tabular}{|l|l|}
\hline FIU IRB Approval: & $10 / 2 / 2013$ \\
\hline FIU IRB Expiration: & $10 / 2 / 2014$ \\
\hline FIU IRB Number: & IRB-13-0416 \\
\hline
\end{tabular}

\title{
BENEFITS
}

The following benefits may be associated with your child's participation in this study: The assessment may yield information regarding the development of imitation and performance on different types of imitation tasks. The participation in the intervention component may lead to an improvement in motor and/or vocal imitation performance.

\begin{abstract}
ALTERNATIVES
There are no known alternatives available to your child other than not taking part in this study. However, any significant new findings developed during the course of the research which may relate to your child's willingness to continue participation will be provided to you. It is possible that participation in other therapies could lead to the development of imitation skills.
\end{abstract}

\section{CONFIDENTIALITY}

Confidentiality for this study will be maintained by using only ID numbers for all measures and video recordings to identify participants. All measures will be kept in a locked file cabinet at the Center for Children and Families at Florida International University. All electronic data will be kept on a network drive in the FIU network physically located in AHC-1 room 237. Although we plan to publish single-subject data it will not be possible to track the responses back to the individuals since only ID numbers will be used. However, records may be reviewed for audit purposes by authorized University or other agents who will be bound by the same provisions of confidentiality.

If we learn about serious harm to you or someone else, we will take steps to protect the person endangered even if it requires telling the authorities without parent permission. If we have reason to believe that a participant is being abused, we will report this to the Florida Abuse hotline. In these instances, we would only disclose information to the extent necessary to prevent harm.

\section{COMPENSATION \& COSTS}

Your child will not receive any compensation for participating in this study. Your child will not be responsible for any costs to participate in this study.

\section{RIGHT TO DECLINE OR WITHDRAW}

Your child's participation in this study is voluntary. Your child is free to participate in the study or withdraw his/her consent at any time during the study. Your child's withdrawal or lack of participation will not affect any benefits to which he/she is otherwise entitled. The investigator reserves the right to remove your child from the study without your consent at such time that they feel it is in the best interest.

\section{RESEARCHER CONTACT INFORMATION}

If you have any questions about the purpose, procedures, or any other issues relating to this research study you may contact: 


\begin{tabular}{|l|l|}
\hline FIU IRB Approval: & $10 / 2 / 2013$ \\
\hline FIU IRB Expiration: & $10 / 2 / 2014$ \\
\hline FIU IRB Number: & IRB-13-0416 \\
\hline
\end{tabular}

Elaine Espanola

DM 269b - Modesto Maidique Campus

11200 S.W. 8 th Street

Miami, FL 33199

(305) 348-4743

eespa001@fiu.edu
Dr. Anibal Gutierrez

DM 269b - Modesto Maidique Campus

11200 S.W. 8th Street

Miami, FL 33199

(305) 348-0042

anibal.gutierrez@fiu.edu

\section{IRB CONTACT INFORMATION}

If you would like to talk with someone about your child's rights of being a subject in this research study or about ethical issues with this research study, you may contact the FIU Office of Research Integrity by phone at 305-348-2494 or by email at ori@fiu.edu.

\section{VIDEOTAPING}

By signing this section you give permission for your child to be videotaped during this study. Please read the following statements regarding use of video/still images and check ALL of the boxes that apply:

[ ] Videos/still pictures may be used for teaching purposes and conference presentations. [ ] Videos/still pictures may be used in the media for study purposes.

Signature of Participant

Signature of person obtaining consent

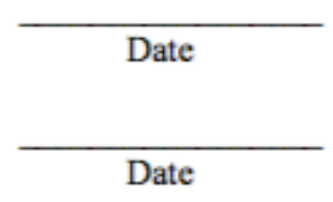

Date

\section{PARTICIPANT AGREEMENT}

I have read the information in this consent form and agree to allow my child to participate in this study. I have had a chance to ask any questions I have about this study, and they have been answered for me. I understand that I am entitled to a copy of this form after it has been read and signed.

Signature of Parent/Guardian

Date

Printed Name of Parent/ Guardian

Printed Name of Child Participant

Signature of Person Obtaining Consent

Date 
Appendix B: Generalization Probes

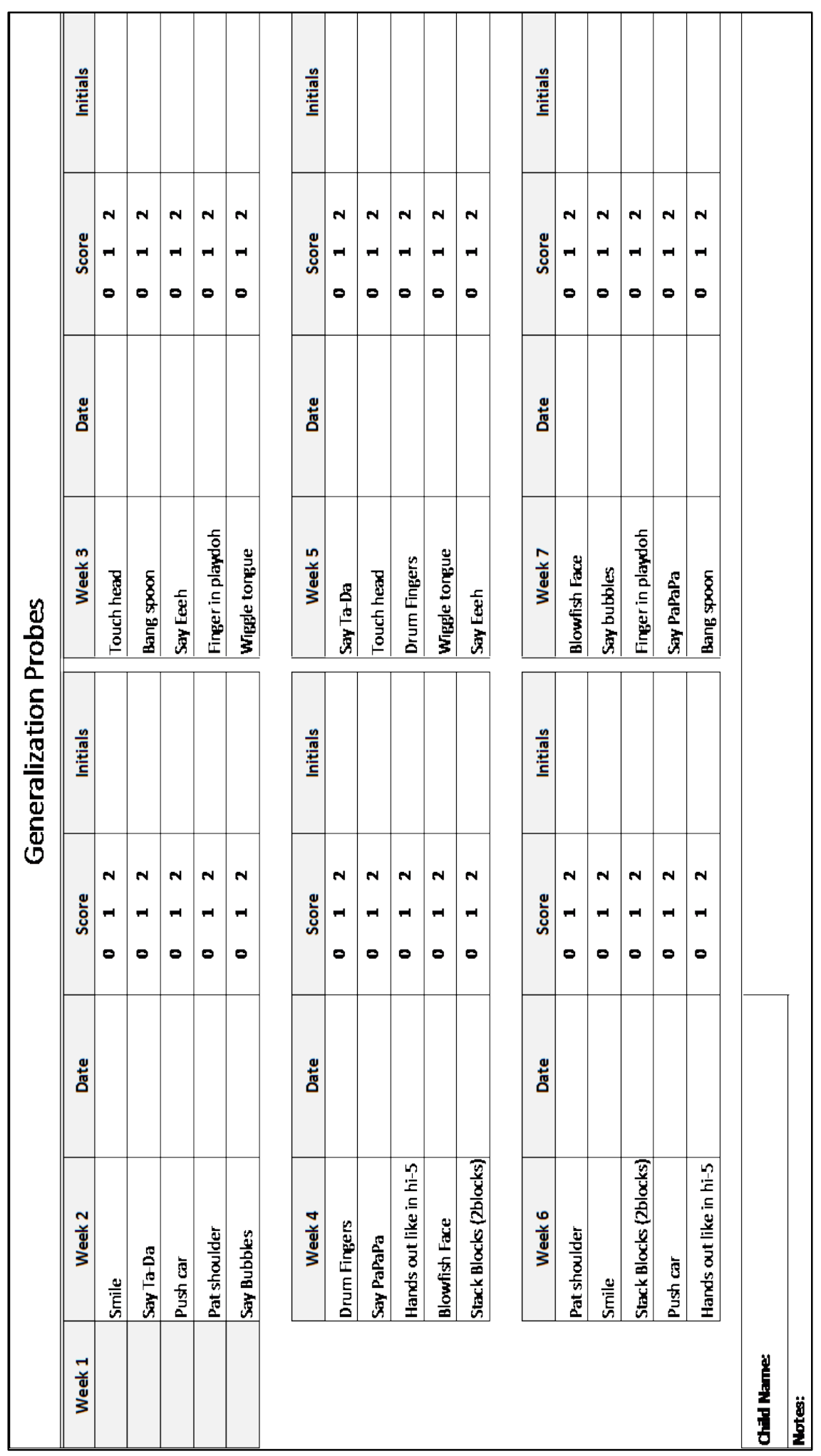


Appendix C: MVIA Protocol \& Mastery Criteria

\section{MASTERY SUMMARY}

\begin{tabular}{|c|c|c|c|}
\hline & \multicolumn{3}{|c|}{ "OBJECT IMITATKN } \\
\hline & & Date Mastered & Trials to Mastery \\
\hline & Meaningful/Noise & & \\
\hline \multirow{4}{*}{$\begin{array}{l}\mathbf{a} \\
\mathbf{0} \\
\mathbf{\xi} \\
\mathbb{\mathbb { W }} \\
\mathbf{x} \\
\end{array}$} & Shake Clapper & & \\
\hline & Squeeze Horn & & \\
\hline & Hops Hard Toy & & \\
\hline & Tums Knob & & \\
\hline & $\begin{array}{l}\text { Imitates } 2 \text { actions with obje } \\
\text { noise. Move down to next } t\end{array}$ & $\begin{array}{l}\text { ts that are mea } \\
\text { grget. }\end{array}$ & ngful and make \\
\hline
\end{tabular}

\begin{tabular}{|r|r|l}
\hline \multicolumn{3}{|c}{ BODY IMTATKN } \\
\hline & Date Mastered & Trials to Mastery \\
\hline Visible/Noise & & \\
\hline Clap Hands & & \\
\hline Bang Hands & & \\
\hline Knocking & & \\
\hline Scratch Table & & \\
\hline
\end{tabular}

Imitates 2 motor movements that are visible (to self) and

make a noise. Move down to next target.

\begin{tabular}{|l|l|l||}
\hline Not-Meaningful/Noise & & \\
\hline
\end{tabular}

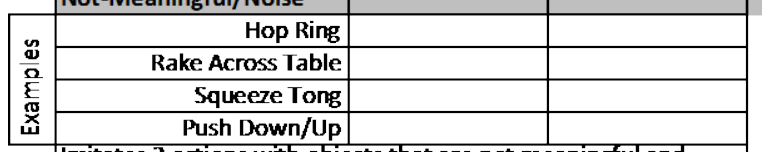

Imitates 2 actions with objects that are not meaningful and

make noise. Move down to next target.

Not-Meaningful/No-Noise

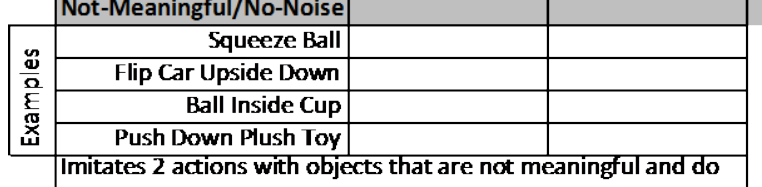

\begin{tabular}{r|l|l}
\hline Not-Visible/No-Noise & & \\
\hline Touches Ear & & \\
\hline Hands Up & & \\
\hline Touches Nose & & \\
\hline Head Side to Side & & \\
\hline
\end{tabular}

Imitates $\mathbf{2}$ motor movements that arenot visible (to self) and

do not make a noise. Move down to next target.

Visible/No-Noise

\begin{tabular}{|r|l|l}
\hline Hand Extend Out & & \\
\hline Hands Together & & \\
\hline Wave Hands & & \\
\hline Open/Ckose Fist & & \\
\hline
\end{tabular}

not make a noise. Move down to next target.

Meaningful/No-Noise

\begin{tabular}{|c|c|c|}
\hline & Meaningful/No-Noise & \\
\hline \multirow{4}{*}{ 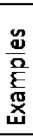 } & Fly Airplane & \\
\hline & Spin Ribbon & \\
\hline & Tum Toy On & \\
\hline & Throw Ball & \\
\hline & $\begin{array}{l}\text { Imitates } 2 \text { actions with obje } \\
\text { make a noise. Move to bod }\end{array}$ & $\begin{array}{l}\text { ects that are meaningful and do not } \\
\text { ly imitation. }\end{array}$ \\
\hline
\end{tabular}

not make a noise. Move down to next target.

\begin{tabular}{|r|l|l}
\hline Not-Visible/Noise & & \\
\hline Bang Hands Under Table & & \\
\hline Clap Hands Above & & \\
\hline Taps Cheek & & \\
\hline Stomp Feet & & \\
\hline
\end{tabular}

Imitates 2 motor movements that are not visible (to self) and make a noise. Move to vocal imitation.

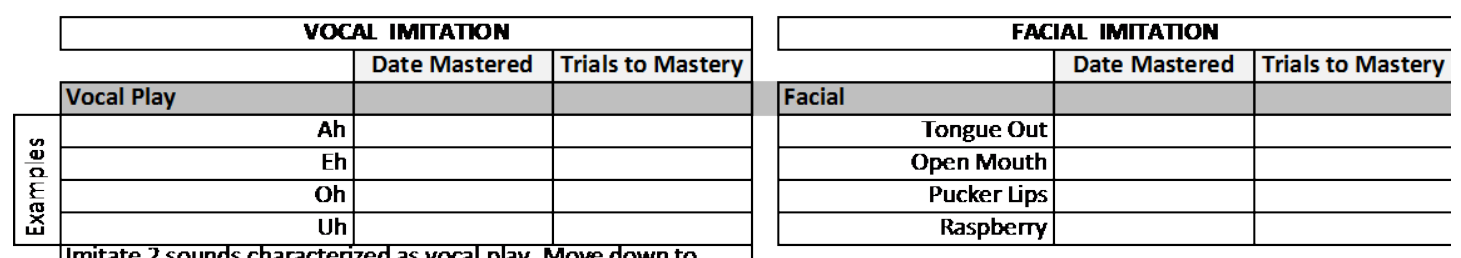

Imitate 2 sounds characterized as vocal play. Move down to next target.

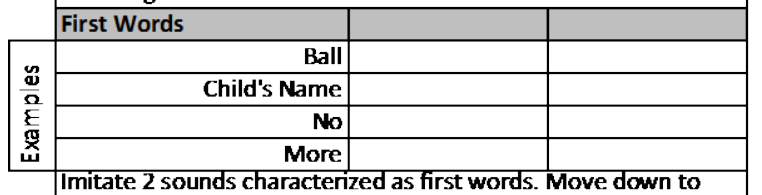

\section{Child Name:}

next target

Non-Reduplicated Speech

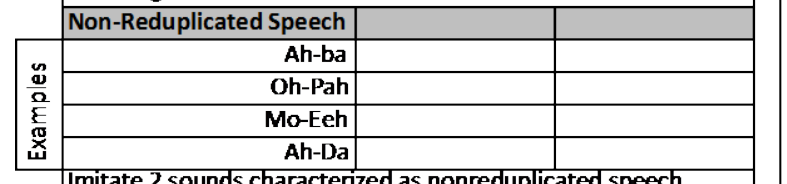

Imitate 2 sounds characterized as nonreduplicated speech.

Move down to next target.

Canonical Babbling

\begin{tabular}{|c|c|c|}
\hline & Canonical Babbling & 1 \\
\hline \multirow{4}{*}{ 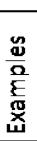 } & Mamam & \\
\hline & DeeDeeDEe & \\
\hline & Dadada & \\
\hline & BuhBuhBuh & 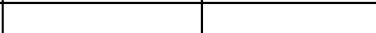 \\
\hline
\end{tabular}

\begin{tabular}{|l|}
\hline Child Name: \\
\hline
\end{tabular}


Appendix D: VB-MAPP Protocol \& Mastery Criteria for Motor Imitation

\begin{tabular}{|c|c|c|}
\hline \multicolumn{3}{|c|}{ Child's Name: } \\
\hline Skill & Motor Imitation Level 1 & Met \\
\hline 1-a & Imitates mouthing movements 3 times (e.g-, Puckering lips, opening mouth) & \\
\hline 1-b & $\begin{array}{l}\text { Imitates } 1 \text { motor movement of another person on demand (i.e., will imitate } \\
\text { when asked) }\end{array}$ & \\
\hline 1-M & $\begin{array}{c}\text { Imitates } 2 \text { gross motor movements when prompted with, Do this (e.g., } \\
\text { clapping, raising arms) }\end{array}$ & \\
\hline 2-a & $\begin{array}{c}\text { Imitates } 2 \text { actions with an object (e.g-, pounding with a toy hammer, rolling a } \\
\text { ball) }\end{array}$ & \\
\hline 2-b & Spontaneously imitates 2 different fun activities (e.g-, patty cake, peek-a-boo) & \\
\hline $2-c$ & Makes eye contact while imitating 3 times & \\
\hline 2-M & Imitates $\mathbf{4}$ gross motor movements when prompted, Do this & \\
\hline 3-a & Imitates 2 fine motor movements (e.g, wiggles fingers, opens and doses fist) & \\
\hline 3-b & $\begin{array}{l}\begin{array}{l}\text { Spontaneously imitates } 2 \text { gross motor movements of another person (e.g., } \\
\text { arms up) }\end{array} \\
\end{array}$ & \\
\hline 3-c & Imitates side-to-side rocking & \\
\hline 3-d & Generalizes known imitation response to 2 additional people & \\
\hline 3-M & $\begin{array}{l}\text { Imitates } 8 \text { motor movemens, } 2 \text { of which involve objects (e.g., shaking a } \\
\text { maraca, tapping sticks together }\end{array}$ & \\
\hline 4-a & Imitates pointing at people or objects & \\
\hline 4-b & Imitates transferring an object from one hand to another & \\
\hline 4-c & $\begin{array}{c}\text { Imitates } 5 \text { actions that produce an outcome (e.g., pushing buttons on pop-up } \\
\text { toy) }\end{array}$ & \\
\hline 4-d & Imitates 2 head movements (e.g., nodding and shaking) & \\
\hline 4-M & Spontaneously imitates the motor behaviors of others on 5 occassions & \\
\hline 5-a & Imitates 5 different fine motor movements (e.g-, pokes clay with index finger) & \\
\hline 5-b & $\begin{array}{l}\text { Imitates the gross motor behavior of other children } 2 \text { times when prompted } \\
\text { (e.g-, running) }\end{array}$ & \\
\hline 5-c & $\begin{array}{l}\text { Spontaneously imitates the behavior of others on } 2 \text { occasions (e.g., filling } \\
\text { containers) }\end{array}$ & \\
\hline 5-d & Demonstrates generalization of 10 imitative behaviors to 3 new people & \\
\hline 5-M & $\begin{array}{l}\text { Imitates } 20 \text { motor movements of any type (e.g., fine motor, gross motor, } \\
\text { imitation with objects) }\end{array}$ & \\
\hline
\end{tabular}


Appendix E: VB-MAPP Protocol \& Mastery Criteria for Vocal Imitation

\begin{tabular}{|l|l|}
\hline & Vocal Imitation \\
\hline Child's Name: & \\
\hline
\end{tabular}

Group 1: Simple and reduplicated syllables
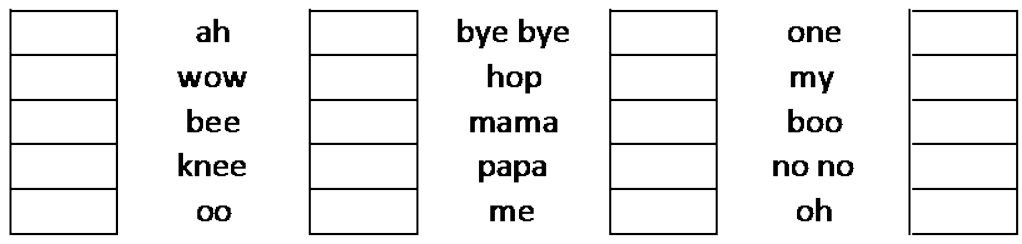
moo up
may
pop
too

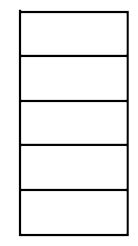

we

boy

wa wa

toy

baa

Group 2: 2-syllable combinations
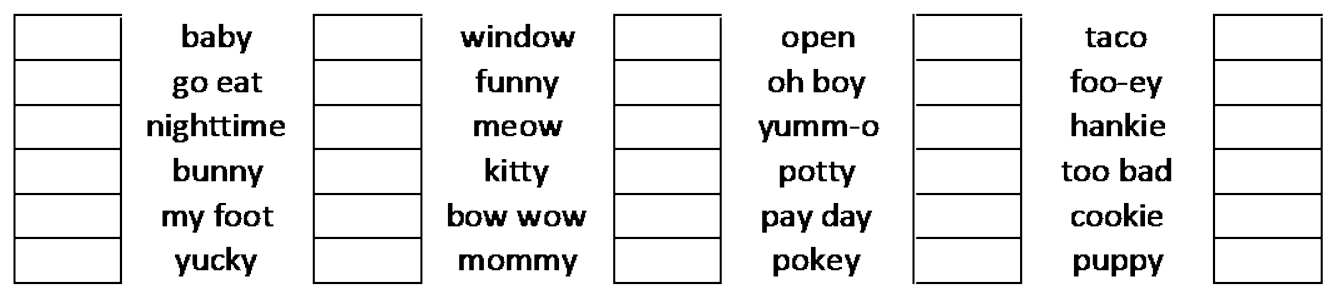

icky too hot monkey uh-oh daddy hot dog

Group 3: 3-syllable combinations

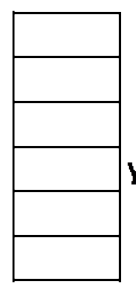

\begin{tabular}{|l} 
tubby toy \\
banana \\
fee fi foe \\
\cline { 2 - 2 } yummy food \\
daddy up \\
in a boat
\end{tabular}

\begin{tabular}{|l|} 
potato \\
go bye bye \\
fat doggy \\
goofy goat \\
hey me too \\
my big toe
\end{tabular}

\begin{tabular}{|l} 
do high five \\
oh foo-ey \\
binky boo \\
one cookie \\
open up \\
peanut hat
\end{tabular}

\begin{tabular}{|c|} 
tiny pan \\
peek a boo \\
teddy bear \\
doggy bone \\
funny king \\
\cline { 2 - 2 } a hiccup
\end{tabular}

how many potty time giddy up wet mitten teepee boat

Group 4: Prosody: spoken phrases

\begin{tabular}{|c} 
no WAY \\
bug-a-BOO
\end{tabular}

ONE bunny
UH-oh

\begin{tabular}{|l|}
\hline \\
in a MINute \\
MY mommy
\end{tabular}

TAKE it bow-WOW my MOMmy

BUG-a-boo 
Appendix F: Treatment Fidelity

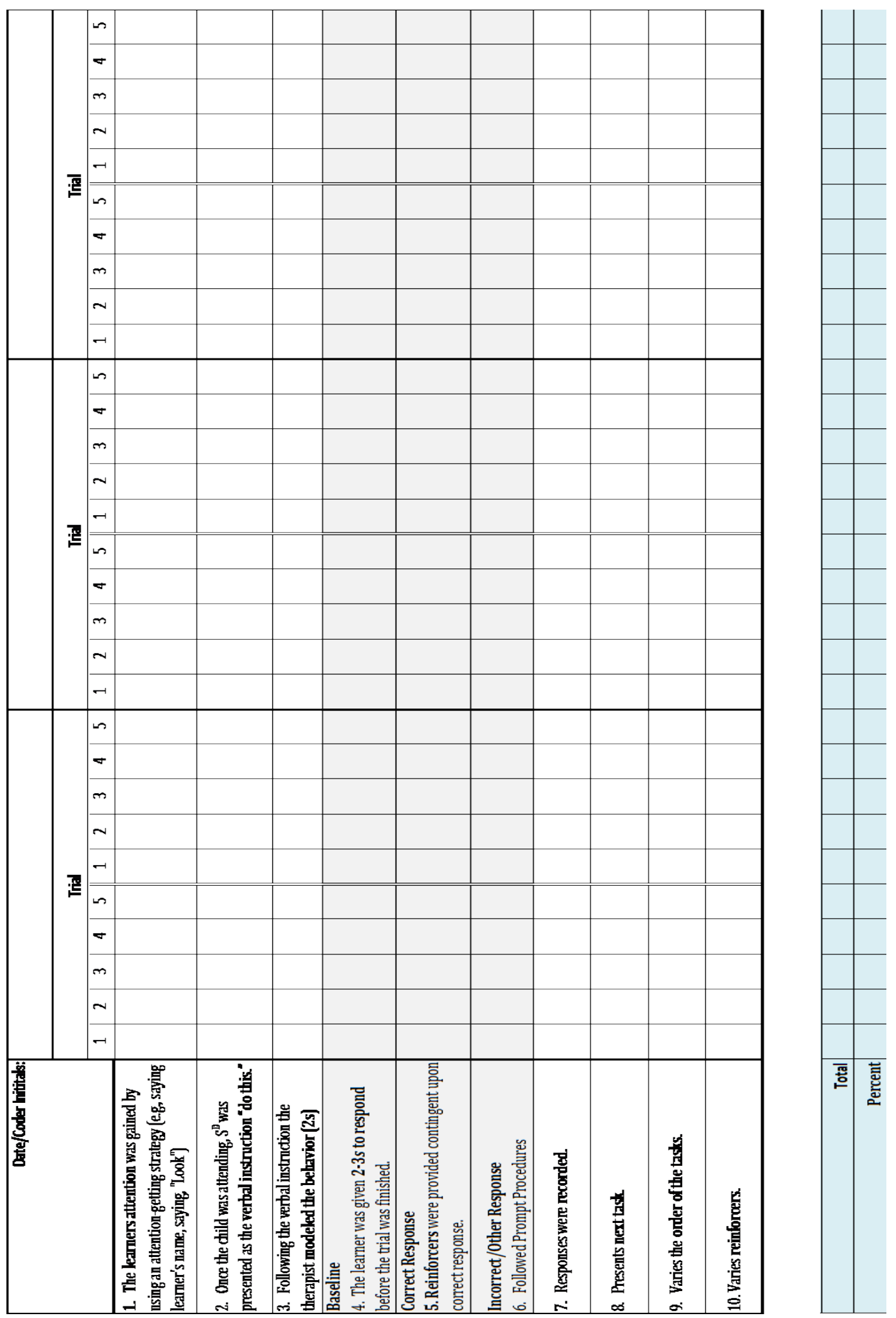


Appendix G: Participant 1 MVIA Treatment Group

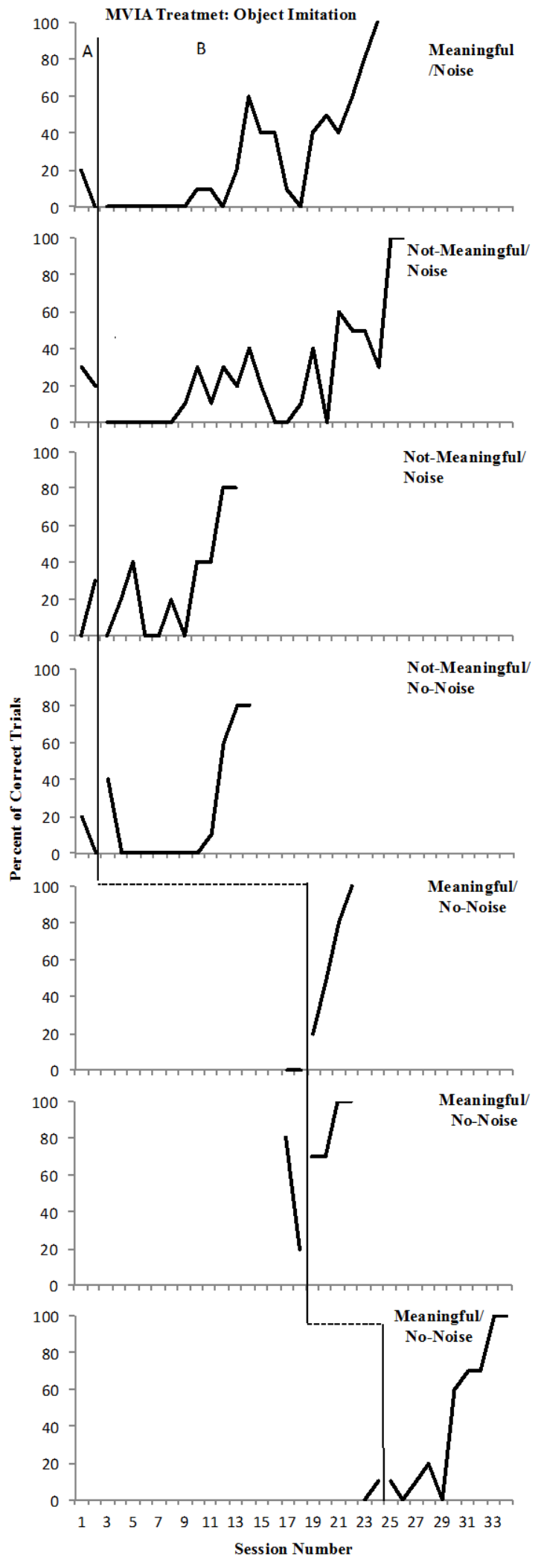




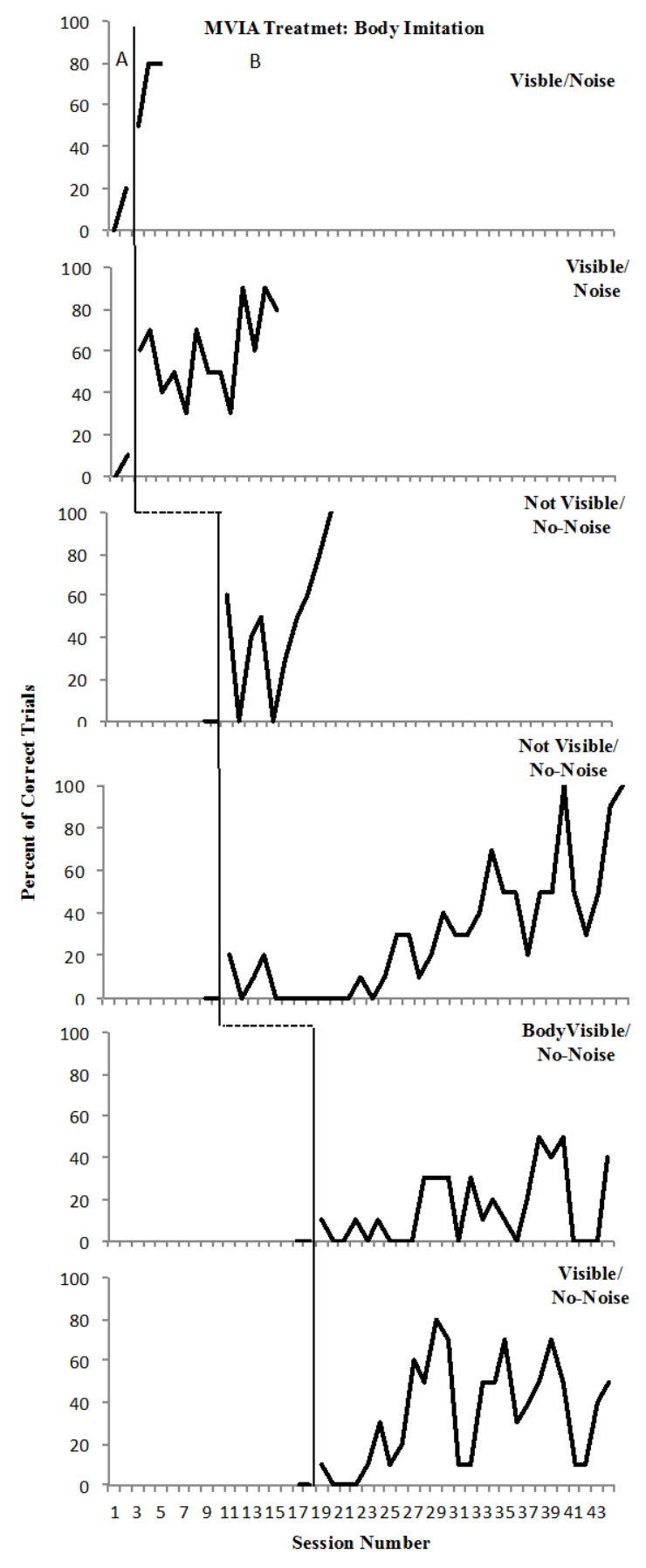


Appendix H: Participant 1 VB-MAPP Comparison Group

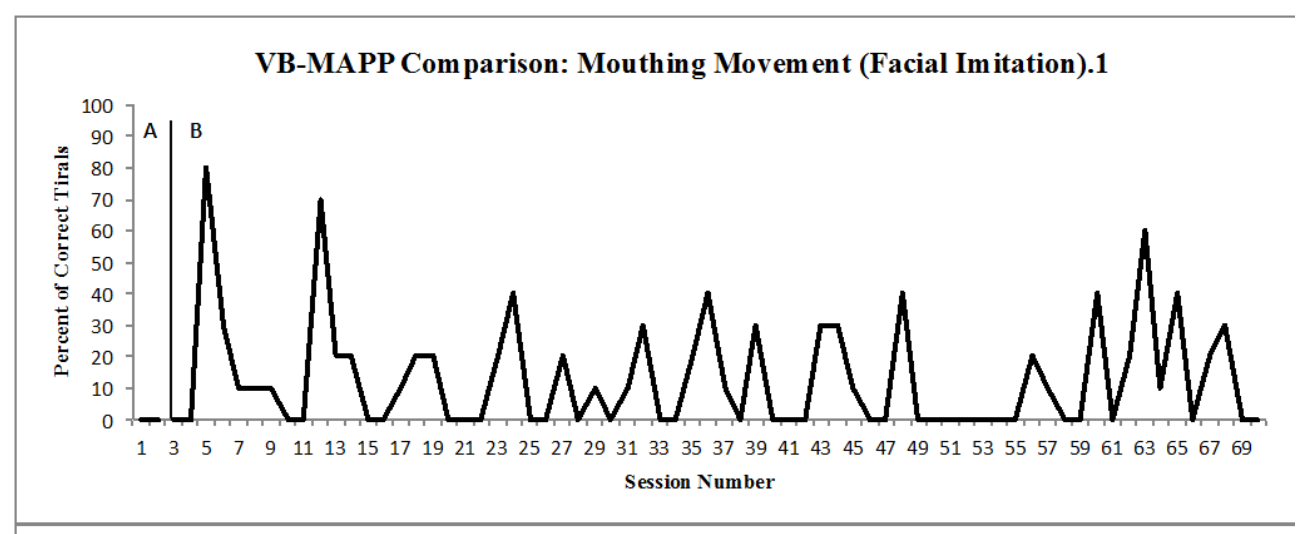

VB-MAPP Comparison: Mouthing Movement (Facial Imitation).2

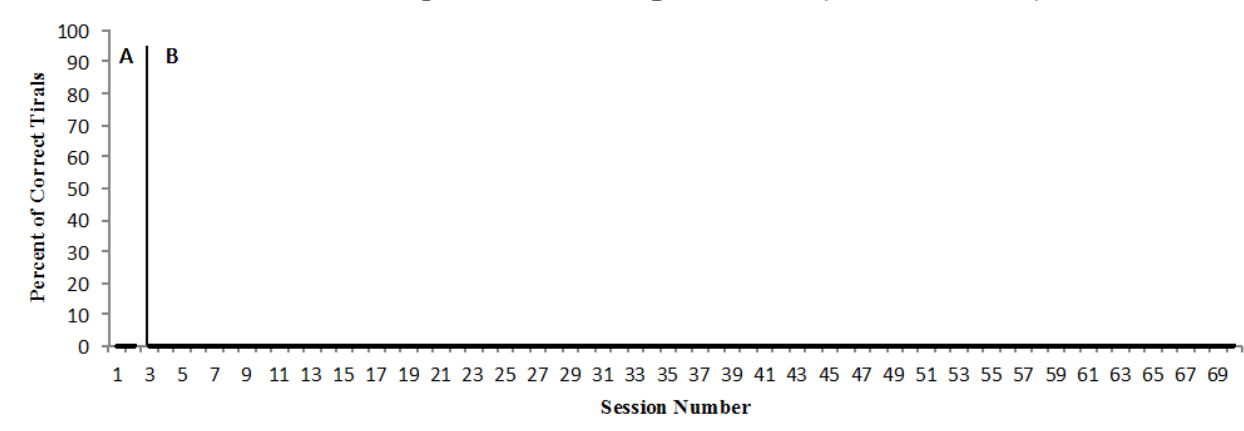

VB-MAPP Comparison: Simple/Reduplucated Syllables (Vocal Imitation).1

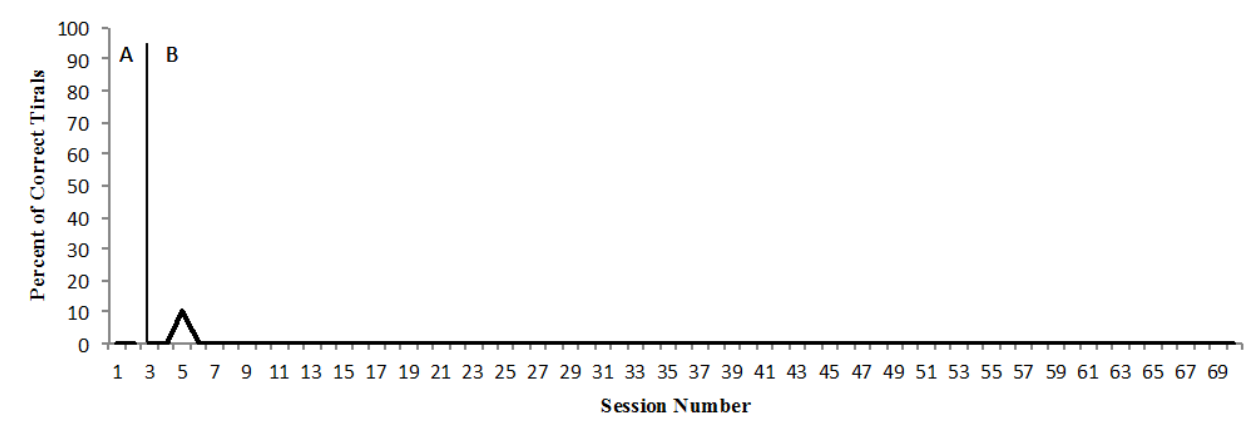

VB-MAPP Comparison: Simple/Reduplucated Syllables (Vocal Imitation).2

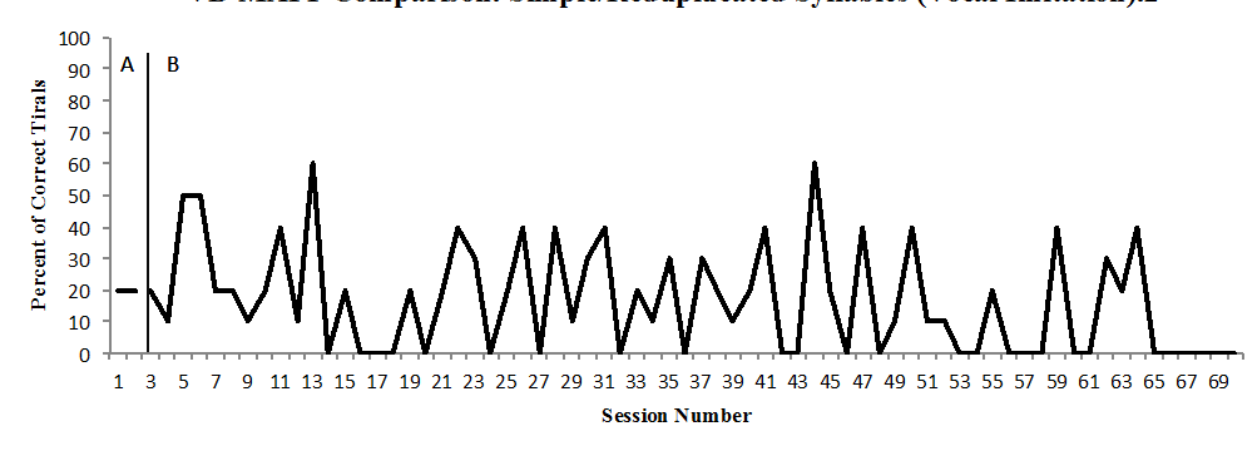


VITA

\section{ELAINE ESPANOLA}

Born, Havana, Cuba

2009

B.A., Psychology

Minor in Sociology/Anthropology

Minor in Marketing

Florida International University

Miami, FL

2012

M.S., Behavior Analysis

Florida International University

Miami, FL

2012

Board Certification in Behavior Analysis

\section{PUBLICATIONS AND PRESENTATIONS}

Elaine, E. \& Gutierrez, A. (2015, October). A Validated Sequence for Selecting Imitation Targets for Intervention. Paper presented at 2014 Florida Association for Behavior Analysis Annual Conference, Daytona Beach, FL.

Espanola, E. (2014, January). Imitation: Development and Its Role in Early Intervention. Paper presented at the 21st Annual Statewide Center for Autism and Related Disabilities Conference, Orlando, FL.

Espanola, E., \& Gutierrez, A. (2014, February). Assessment of Motor and Vocal Imitation. Paper presented at McKnight Doctoral Fellow Mid-Year Research and Writing Conference, Tampa, FL.

Espanola, E., Gutierrez, A., Cruz, V., Aguilar, L., \& Osorio, G. (2014, May). Assessment of Motor and Vocal Imitation. Poster presented at the 40th Annual Association for Behavior Analysis International Convention, Chicago, IL.

Espanola, E \& Gutierrez, A. (2014, September). Assessment of Motor and Vocal Imitation. Paper presented at 2014 Florida Association for Behavior Analysis Annual Conference, Bonita Springs, FL. 